2018-07-03

\title{
Sensor Fusion-based Framework for Floor Localization
}

\author{
Haque, Fahimul
}

Haque, F. (2018). Sensor Fusion-based Framework for Floor Localization (Unpublished master's thesis). University of Calgary, Calgary, AB. doi:10.11575/PRISM/32261

http://hdl.handle.net/1880/107039

Downloaded from PRISM Repository, University of Calgary 


\title{
UNIVERSITY OF CALGARY
}

Sensor Fusion-based Framework for Floor Localization

by

\author{
Fahimul Haque
}

\begin{abstract}
A THESIS
SUBMITTED TO THE FACULTY OF GRADUATE STUDIES

IN PARTIAL FULFILMENT OF THE REQUIREMENTS FOR THE

DEGREE OF MASTER OF SCIENCE
\end{abstract}

GRADUATE PROGRAM IN ELECTRICAL AND COMPUTER ENGINEERING

CALGARY, ALBERTA

JULY, 2018

(C) Fahimul Haque 2018 


\section{Abstract}

Floor localization is at the heart of indoor positioning systems (IPSs) in multi-storey buildings with a variety of commercial, industrial, and health and safety applications. The prevalence of wireless technologies along with the integration of micro electro-mechanical sensors (e.g. barometers) in handheld devices and wearable gadgets of current vintage have prompted a surge in research and development efforts in the IPS area. Received signal strength (RSS), barometric altimetry (BA), and differential barometric altimetry (DBA) are three well-known methods of floor localization. However, the RSS-based methods lack the required accuracy, BA-based methods are prone to random errors due to local changes in the air pressure, e.g. from approaching weather systems, and DBA-based methods require installation of additional infrastructure (e.g. reference nodes and ad-hoc network for realtime information exchange). Fusion of BA and RSS is a viable solution for floor localization; nevertheless, available fusion algorithms are rather heuristic. In this dissertation, a theoretical framework is developed for fusing BA and Wi-Fi RSS measurements. The proposed framework involves a novel Monte Carlo Bayesian inference algorithm, for processing RSS measurements, and then fusion with BA using a Kalman Filter scheme. As demonstrated by our experimental results, the proposed sensor fusion algorithm achieves floor localization accuracy of $97 \%$ on average. The algorithm does not require new infrastructure, and has low computational complexity, hence, can be readily integrated into various state-of-the-art mobile devices. 


\section{Acknowledgements}

I would like to extend my heartfelt gratitude to my supervisor Dr. Vahid Dehghanian and my co-supervisor Dr. Abraham Fapojuwo for their constant assistance, guidance, and encouragement during my MSc program. Thank you for providing your valuable insights and expertise to support me with my research work.

I would also like to thank my parents and my brothers who have always been there for me. 


\section{Dedication}

To my amazing family, beloved fiancé, and lighthearted friends. 


\section{Table of Contents}

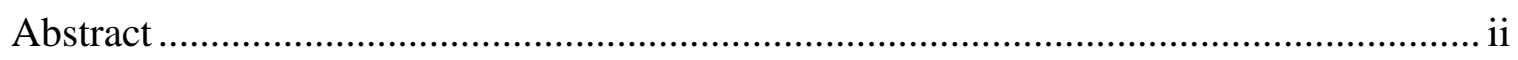

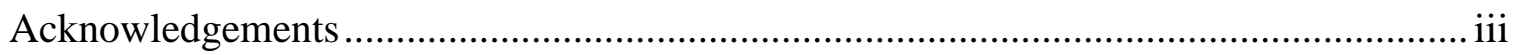

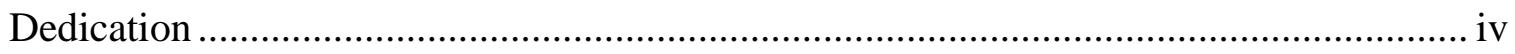

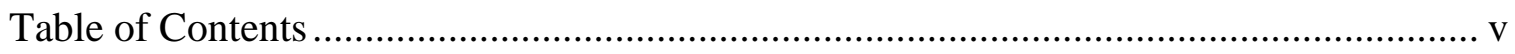

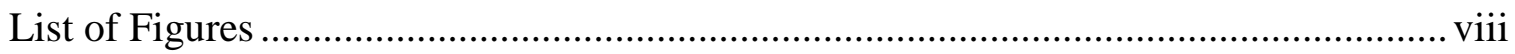

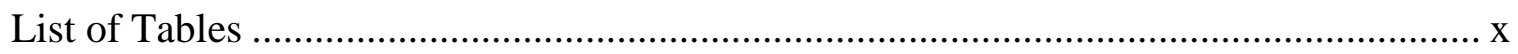

List of Symbols, Abbreviations and Nomenclature ......................................................... xi

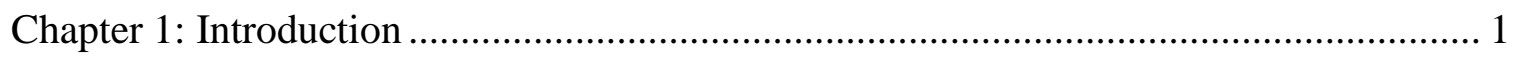

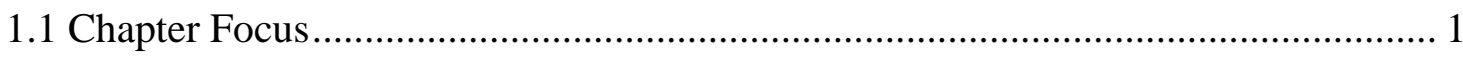

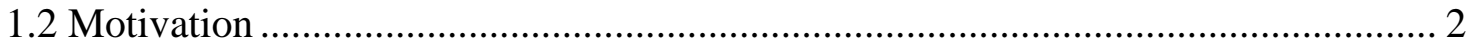

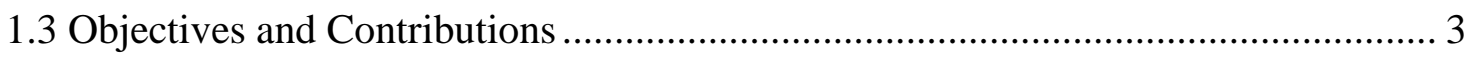

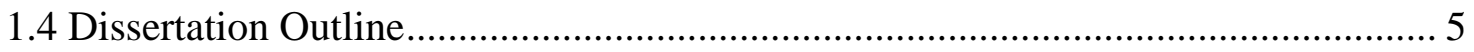

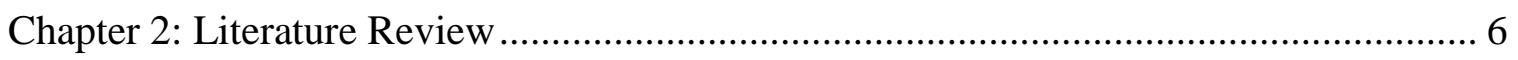

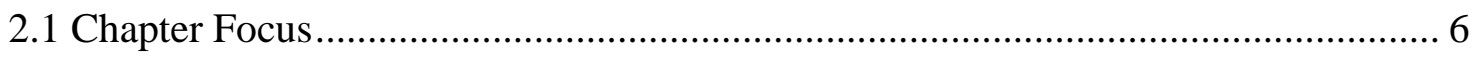

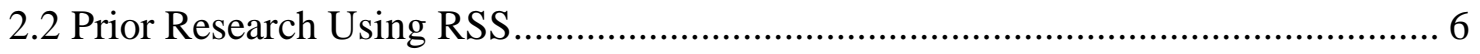

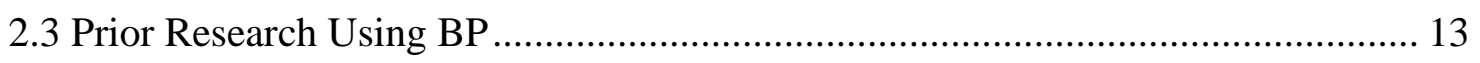

2.4 Prior Research Using Both RSS and BP ......................................................... 18

2.5 Relationship between the Thesis and Previous Works............................................. 21

Chapter 3: Floor Localization Using Barometric Pressure …………………................ 22

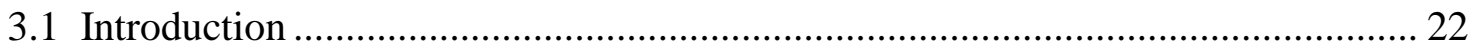

3.2 MEMS Barometer Measurement Model ............................................................. 23 
3.3 Framework for BP-based Floor Localization...................................................... 30

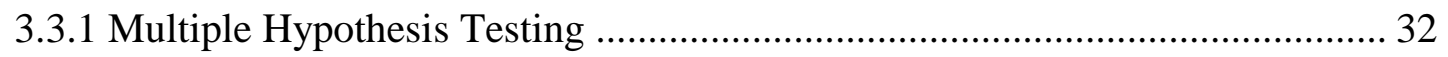

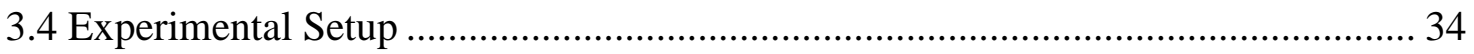

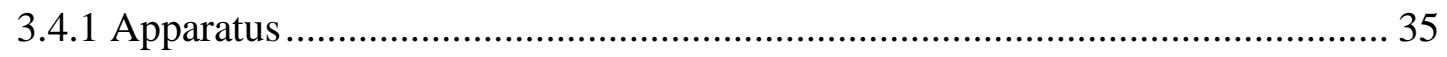

3.4.2 Selected Environments for Measurements ....................................................... 36

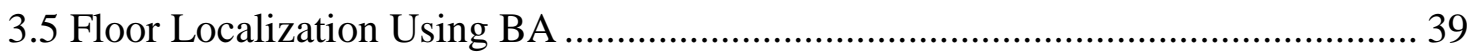

3.5.1 Results and Analysis of BA-based Floor Localization..................................... 40

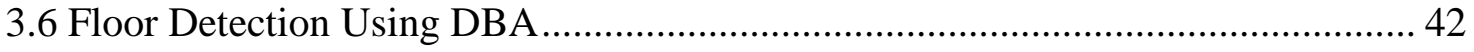

3.6.1 Results and Analysis of DBA-based Floor Localization................................... 44

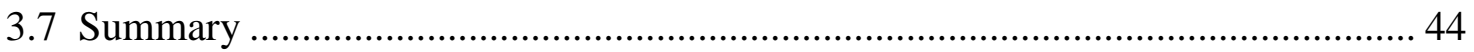

Chapter 4: Floor Localization Using Received Signal Strength ...................................... 47

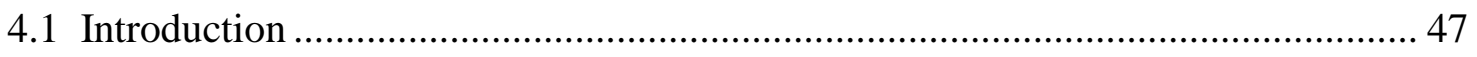

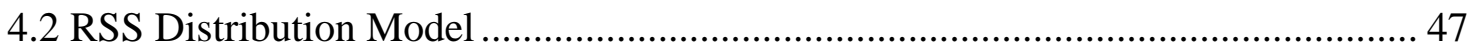

4.3 Conventional RSS-based Floor Localization Methods ........................................... 53

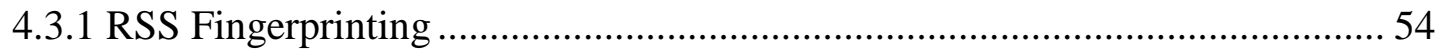

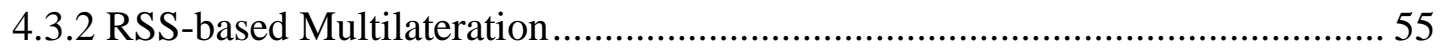

4.3.3 Maximum Likelihood-based Approach .......................................................... 56

4.4 Proposed MCBI Framework for RSS-based Floor Localization ............................ 57

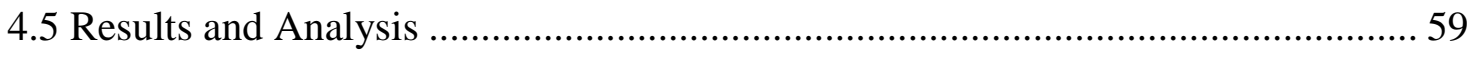

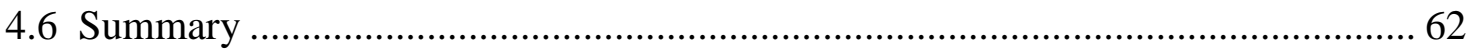

Chapter 5: Floor Localization Using Proposed Sensor Fusion Framework ..................... 63

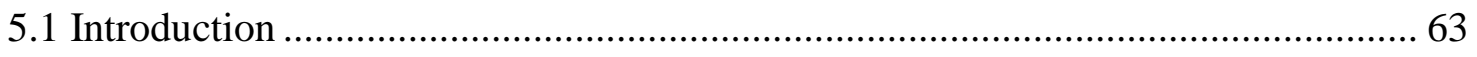

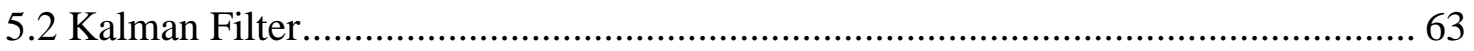


5.3 Proposed Sensor Fusion Algorithm................................................................ 66

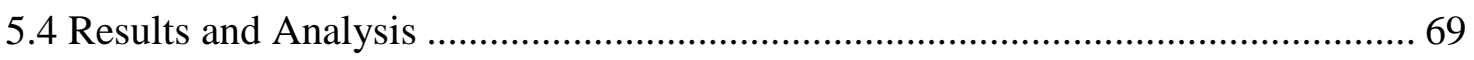

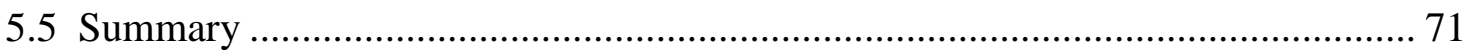

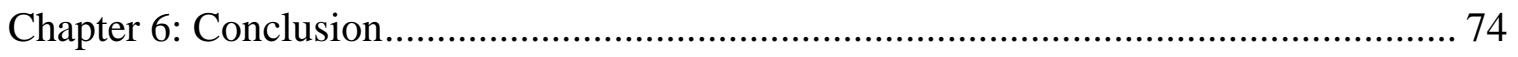

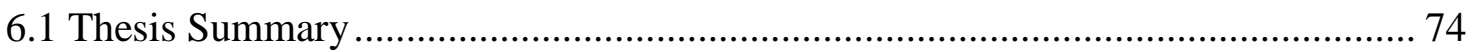

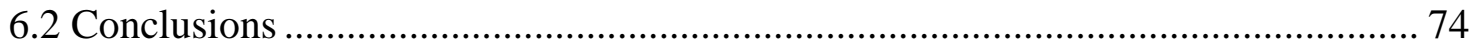

6.3 Recommendations for Future Work ........................................................ 75

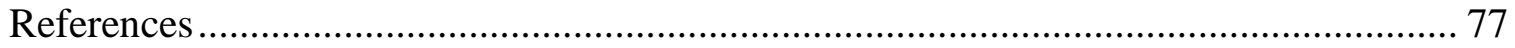

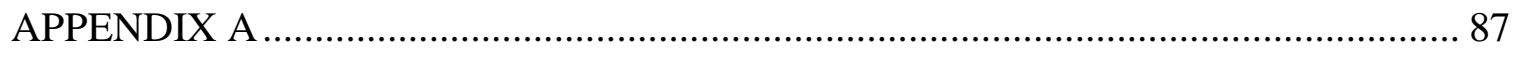

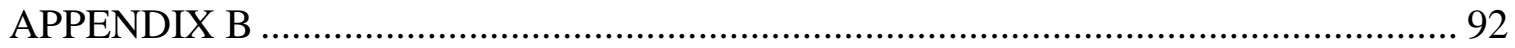

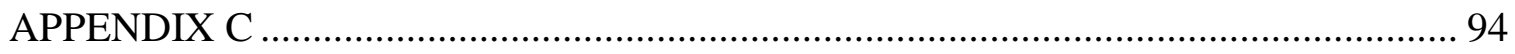




\section{List of Figures}

Figure 3.1: Determined height error $\left(\Delta_{h}\right)$ for 115 hours long measurement set .......... 24

Figure 3.2: Allan deviation plot for MEMS barometer of different models ............... 26

Figure 3.3: Allan deviation plots for MEMS BP sensors (DBA) ...................... 27

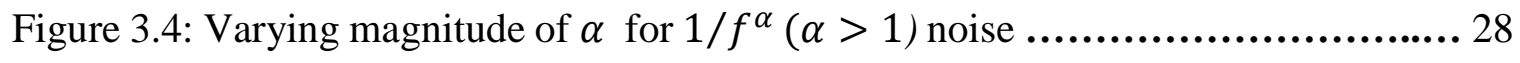

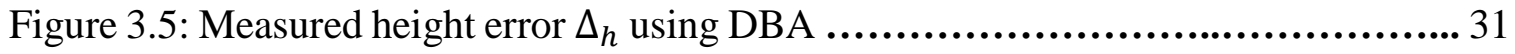

Figure 3.6: Distribution of estimated height $\hat{h}^{b}$ on a fixed floor (First floor of TFDL) ... 31

Figure 3.7: Handheld electronic comprising BMP280 and Wi-Fi module ................ 35

Figure 3.8: Left figure - Outside view of ICT building; Middle figure - Narrow hallway on the second/third floor; Right figure - Partial view of the first floor (open floor plan) . 37

Figure 3.9: Top figure - Outside view of TFDL building; Middle figure - Hollow interiors between first and second floor; Bottom figure - Partial view of the third floor ........... 37

Figure 3.10: Top figure - Outside view of MSC; Middle figure - Hollow interiors inside MSC; Bottom Figure - Open floor plan on the first floor of MSC ...................... 38

Figure 3.11: BA-based floor localization system ..................................... 39

Figure 3.12: Estimated height using BA in (a) ICT, (b) TFDL, and (c) MSC ............ 41

Figure 3.13: Distribution of estimated height $\hat{h}^{b}$ (1.5 hours apart) on the first floor

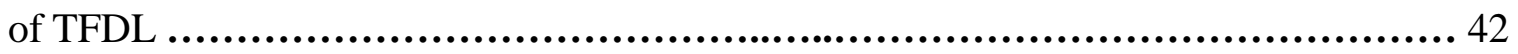

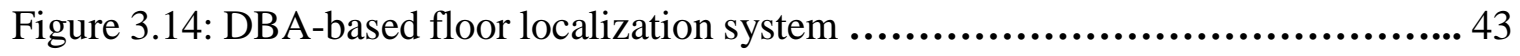

Figure 3.15: Estimated height using DBA in (a) ICT, (b) TFDL, and (c) MSC ........... 45

Figure 3.16: Distribution of estimated height $\hat{h}^{b}$ using DBA on different floors

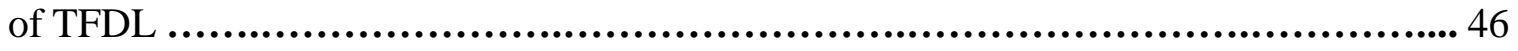

Figure 4.1: Illustration of multipath effect in an indoor environment ................... 48 
Figure 4.2: Distribution of RSS measurements form an AP with fixed transmitter-receiver

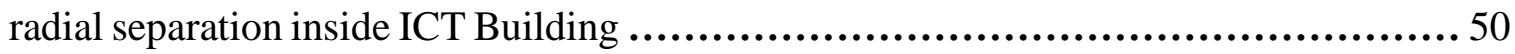

Figure 4.3: (a) Distribution of $\operatorname{RSS}\left(\left|t_{m}-l_{f}\right|=0\right)$, (b) Distribution of RSS

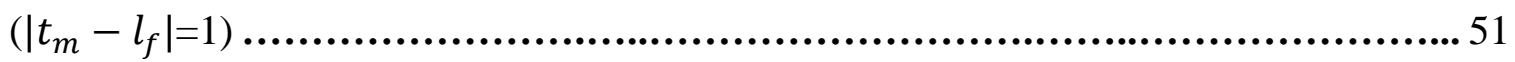

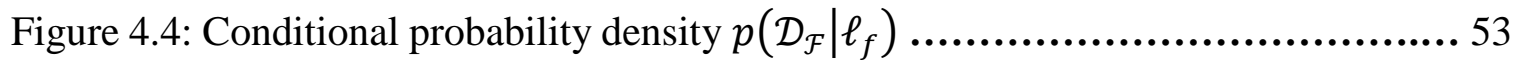

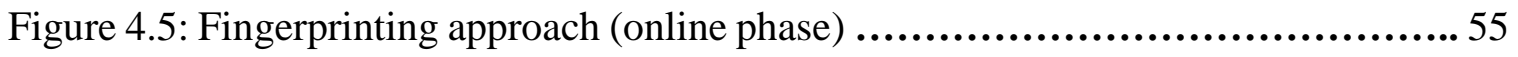

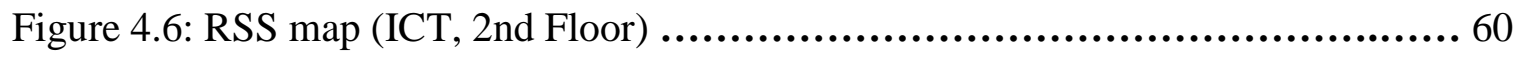

Figure 4.7: Impact of AP availability on floor localization ........................... 61

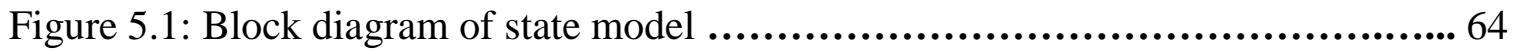

Figure 5.2: Block Diagram of the Kalman filter ..................................... 66

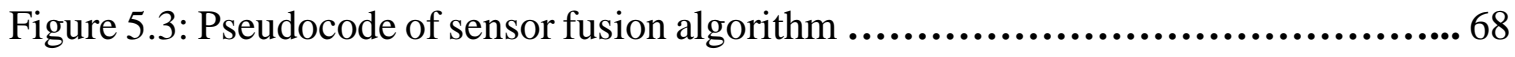

Figure 5.4: Estimated height for floor localization using sensor fusion algorithm in (a) ICT,

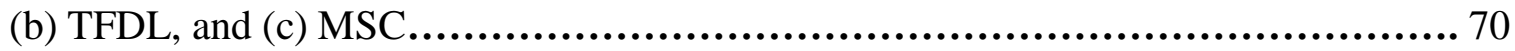

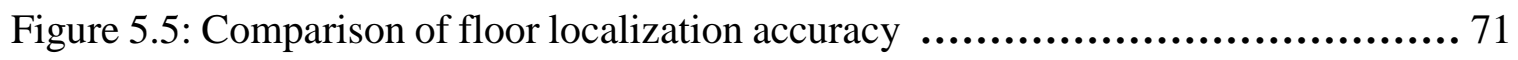

Figure 5.6: Percentage of false floor localization in (a) ICT, (b) TFDL, and (c) MSC .... 72

Figure C.1: Average RSS measurements .......................................... 95

Figure C.2: Distribution of RSS on different floors (signals transmitted by AP 1) ....... 96 


\section{List of Tables}

TABLE 3.1 NOISE CHARACTERISTICS OF THE TESTED BP SENSORS ......... 28

TABLE 3.2 FLOOR LOCALIZATION ACCURACY USING BA ..................... 42

TABLE 3.3 FLOOR LOCALIZATION ACCURACY USING DBA .................... 46

TABLE 4.1 STATISTICAL CHARACTERISTICS OF LOG-NORMAL

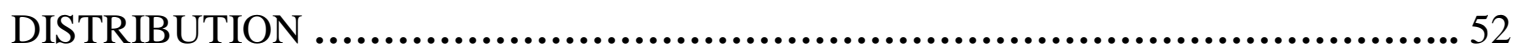

TABLE 4.2 FLOOR LOCALIZATION ACCURACY USING RSS-BASED

ALGORITHMS ........................................................................ 61

TABLE 5.1 FLOOR LOCALIZATION ACCURACY USING SENSOR FUSION

ALGORITHM ........................................................................... 69

TABLE A.1 ESTIMATED AR(1)MA(1) PARAMETERS ........................... 91

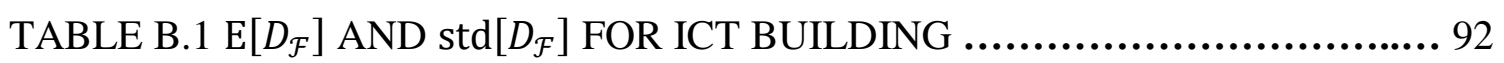

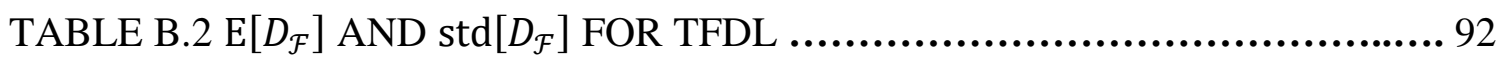

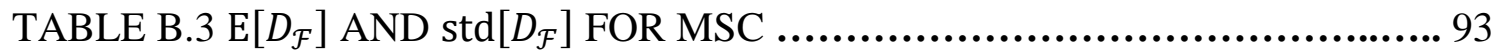




\section{List of Symbols, Abbreviations and Nomenclature}

\section{Symbols}

$a_{n}$

$b[n]$

$b_{k}$

C

$\mathcal{C}$

$\mathcal{D}$

$d_{m}$

$\mathcal{D}_{\mathcal{F}}$

$d_{j, i}$

$\eta$

$\mathcal{F}$

$F_{S}$

$h_{m}$

$\Delta_{h}$

$\tilde{h}$

$h$

\section{Definition}

Amplitude of the $n$-th impulse

Randomly varying mean (i.e. bias) in barometric pressure at n-th sampling interval

Randomly varying mean (i.e. bias) in barometric pressure for $n=(k-1) N_{b}+1, \ldots, k N_{b}$-th sampling intervals, where $k=1,2,3$.. and $N_{b}=F_{S} T_{b}$

Assigned cost

Total number of clusters for a given cluster time in Allan deviation

Observed data during a Wi-Fi network scan

Measurement set that includes $z_{m}$ and $t_{m}$

Observed data for a given mobile-access point floor separation $\mathcal{F}$

Euclidean distance between $j$-th access point and $i$-th measurement point

Path loss coefficient

Floor separation between mobile and access point (i.e. $\mid t_{m}-$ $\left.\ell_{f} \mid\right)$

Sampling frequency

Calculated height using atmospheric pressure with reference to sea level

Height error in measurement

Measured height relative to a local reference point

Correct height relative to the local reference point 


\begin{tabular}{|c|c|}
\hline$h_{r}$ & $\begin{array}{l}\text { Height of the reference point/floor location with reference } \\
\text { to sea level }\end{array}$ \\
\hline $\bar{h}_{f}$ & $\begin{array}{l}\text { Correct floor height associated with floor hypothesis } \ell_{f} \text { with } \\
\text { respect to local reference point }\end{array}$ \\
\hline$\widehat{h} \mid b_{k}, \hat{h}^{b}$ & Estimated height for a known bias $b_{k}$ \\
\hline$h_{R S S}$ & $\begin{array}{l}\text { The correct floor height corresponding to a floor } \\
\text { (determined using RSS-based MAP classifier) }\end{array}$ \\
\hline$h_{p q}(t ; \tau)$ & $\begin{array}{l}\text { Time-varying impulse response of the channel between } \\
\text { nodes } p \text { and } q \text { at time } t \text { to a unit impulse } \delta[.] \text { transmitted at } \\
\text { time } t-\tau\end{array}$ \\
\hline$K$ & Kalman Gain \\
\hline $\mathcal{L}$ & $\begin{array}{l}\text { Set of all possible user floor locations in an } N \text {-storey } \\
\text { building }\end{array}$ \\
\hline $\mathcal{L}^{\mathrm{s}}$ & $\begin{array}{l}\text { Subset of floors that are associated with the access points } \\
\text { discovered during a wireless network scan }\end{array}$ \\
\hline$\ell_{f}$ & Hypothesis associated with $f$-th floor \\
\hline$\ell_{\text {MAP }}$ & Output (i.e. detected floor) of the MHT algorithm \\
\hline$\ell_{\mathrm{MAP}}^{R}$ & Output (i.e. detected floor) of the RSS-based MAP classifier \\
\hline$M$ & Total number of measurement samples \\
\hline $\mathcal{M}$ & Minimum mean square error \\
\hline$N$ & Total number of floors inside a building \\
\hline$N_{\mathrm{s}}$ & $\begin{array}{l}\text { Total number of floors associated with the access points } \\
\text { discovered during a wireless network scan }\end{array}$ \\
\hline$N_{c}$ & Number of samples per cluster in Allan deviation \\
\hline$n$ & Sample/iteration Number \\
\hline$n^{A P}$ & Total number of observed access points during a Wi-Fi scan \\
\hline
\end{tabular}




\begin{tabular}{|c|c|}
\hline $\mathcal{P}$ & $\begin{array}{l}\text { Barometric pressure measured by the pressure sensor (i.e. } \\
\text { barometer) }\end{array}$ \\
\hline $\mathcal{P}_{o}$ & Pressure at sea level, which is $1013.25 \mathrm{hPa}$ or $101325 \mathrm{~Pa}$ \\
\hline $\mathcal{P}_{r}$ & Pressure of the reference location with reference to sea level \\
\hline$p$ & Probability density function or likelihood function \\
\hline$P$ & Probability \\
\hline$P_{e}$ & Probability of error \\
\hline$P_{r_{i}}$ & Received signal strength at $i$-th measurement point \\
\hline$P_{T_{j}}$ & Transmitted power by $j$-th access point \\
\hline$\phi_{\mathcal{F}}$ & $\begin{array}{l}\text { Percentage of discovered access points for a given floor } \\
\text { separation } \mathcal{F}\end{array}$ \\
\hline$\phi_{n}$ & Phase of the $n$-th impulse \\
\hline $\mathcal{R}$ & Bayes risk \\
\hline$s_{p q}$ & Transmitted signal \\
\hline$\sigma_{A D E V}$ & Allan deviation \\
\hline$\sigma_{b}, \sigma_{u}, \sigma_{w}$ & Standard deviation of WGN \\
\hline$T_{b}$ & $\begin{array}{l}\text { Time interval over which the WGN is the dominant noise } \\
\text { process in barometer measurement }\end{array}$ \\
\hline$t_{m}$ & Floor location corresponding to $m$-th AP \\
\hline$\tau_{c}$ & Cluster time (averaging time) in Allan deviation \\
\hline$\tau_{n}$ & Time-delay of the $n$-th impulse \\
\hline$v_{p q}$ & $\begin{array}{l}\text { Induced voltage across terminals of the antenna on the } \\
\text { receiver side }\end{array}$ \\
\hline$\omega_{f}$ & Normalized importance weights \\
\hline$z$ & RSS observables from an access point \\
\hline$z_{m}$ & Measured RSS value (in $\mathrm{dBf}$ ) from $m$-th AP \\
\hline
\end{tabular}


$Z^{T P}$

$Z^{O P}$

$\mathbb{Z}^{+}$
Set of RSS observables at the $i$-th location during training phase

Set of RSS observables during online phase

Set of positive integers 


\section{Abbreviations}

AOA

ADEV

AP

BA

BP

DBA

ED

GNSS

ICT

IPS

KF

KNN

LOS

MAC ID

MAP

MCBI

MEMS

MHT

ML

MSC

MSE

PDF

PSD

\section{Definition}

Angle of Arrival

Allan Deviation

Access Point

Barometric Altimetry

Barometric Pressure

Differential Barometric Altimetry

Euclidean Distance

Global Navigation Satellite System

Information and Communications

Technology Building, University of Calgary

Indoor Positioning System

Kalman Filter

$k$-nearest Neighbor (algorithm)

Line-of-sight

Medium Access Control Identifications

Maximum Posteriori Probability

Monte Carlo Bayesian Inference

Micro-Electro-Mechanical-Systems

Multiple Hypothesis Testing

Maximum Likelihood

MacEwan Student Centre, University of Calgary

Minimum Squared Error

Probability Density Function

Power Spectral Density 


$\begin{array}{ll}\text { RW } & \text { Random Walk } \\ \text { RN } & \text { Reference Node } \\ \text { RSS } & \text { Received Signal Strength } \\ \text { SUT } & \text { Sensor Under Test } \\ \text { TOA } & \text { Time of Arrival } \\ \text { TDOA } & \text { Time Difference of Arrival } \\ \text { TFDL } & \text { Taylor Family Digital Library, University of } \\ & \text { Calgary } \\ \text { WGN } & \text { White Gaussian Noise }\end{array}$




\section{Chapter 1: Introduction}

\subsection{Chapter Focus}

The prevalence of wireless technologies and the integration of miniaturized sensors in modern gadgets, e.g. smartphones and wearable electronics, has prompted a recent surge in mobile location based services (LBS). The total LBS revenues are supposed to reach US\$ 3.8 billion in North America and €2.3 billion in Europe by 2018 [1]. As a core technology of LBS, indoor positioning system (IPS) is vital to detect a smartphone user location in indoor environment. There is a high demand for robust IPS because most LBS users spend $70 \%-90 \%$ of their time indoors [1]. Application areas of IPS are gradually expanding, ranging from goods and services to search and rescue $[2,3]$.

In multi-story buildings, floor localization (also known as floor detection/identification) is an essential part of IPS. In fact, the Federal Communication Commission in USA emphasized on developing solutions for estimating altitude so that the floor location of the emergency (911) callers can be determined [4]. With the ubiquitous use of wireless access points (APs), e.g. Wi-Fi APs, and the integration of Micro-electromechanical-systems (MEMS) sensors, such as barometric pressure sensors (also known as pressure altimeter or barometer), in modern handheld devices (e.g. smartphones) and wearable gadgets, Wi-Fi received signal strength (RSS) and barometric altimetry (BA) have become two well-known methods for floor localization. The focus of this dissertation is to develop a sensor fusion based framework for enhanced floor localization using Wi-Fi RSS and BA.

This chapter introduces the motivation and objectives behind the research work of this dissertation as well as highlights the contributions of the research to the scientific community. Section 1.2 discusses the motivation for this dissertation, which leads to Section 1.3 where the objectives and contributions of the research work are detailed. Finally, Section 1.4 provides an outline of the remaining chapters in this dissertation. 


\subsection{Motivation}

RSS-based localization techniques have become prominent in recent years [2, 3] because they are inexpensive and readily available. Nevertheless, RSS-based positioning is generally prone to a variety of errors due to for example; multipath fading, signal blocking, and changes in smartphone antenna polarization and pattern from user interaction with the handheld device [5, 6, 7]. RSS-based localization techniques based on crowdsourced real-time RSS fingerprints have been shown to achieve meter-level accuracy $[8,9]$. Nonetheless, crowdsourcing requires new infrastructure and localization based on off-line RSS fingerprints is inaccurate and unreliable especially in multi-story buildings with irregular structures, e.g. floors with hollow interiors.

Recent integration of MEMS barometers in smartphones (e.g. iPhone 6/7/8/X, Samsung note 6/7/8, Google Pixel 2) has prompted its use in relative height (known as altitude) estimation and floor localization [10, 11, 12, 13]. Nevertheless, BA is highly affected by local environmental elements such as approaching high/low pressure weather systems, air-conditioning, wind, temperature, factory calibration (or the lack of), etc. These local variations (i.e. drift) in the air pressure can result in large altitude estimation errors of tens of meters over the course of a few hours [11, 12]. Differential barometric altimetry (DBA) based on (near-) real-time differencing of measured air pressures from mobile and reference barometers, where the altitude of the reference is known, has been shown to achieve decimeter level accuracy $[10,14,15]$. However, DBA requires additional infrastructure, e.g. installation of reference nodes and ad-hoc network for real-time information exchange, hence not readily applicable.

Multi-sensor altimetry based on fusing alternate altitude observables with those obtained from MEMS barometers can be very effective. One such alternate source of altitude observables is Global Navigation Satellite System (GNSS) [11, 13, 16]. Unfortunately, GNSS signals are prone to significant RF penetration losses and multipath, therefore GNSS-barometer fusion is not suitable for indoor positioning. Multi-sensor altimetry based on integrating MEMS barometer and MEMS Inertial Measurement Units (IMUs) has been shown to result in improved altitude estimation [17, 18, 19]. Nevertheless, IMUs and barometers are both prone to drift errors (of different origins) and long-term (i.e. 
$\geq 30$ minutes) reliability of such solutions have not yet been verified. Integration of ultra high frequency based techniques that use time of arrival (TOA), time difference of arrival (TDOA), and angle of arrival (AOA) is not feasible for IPS because they require sophisticated and costly devices, e.g. ultra-high precision clocks for TOA, TDOA or antenna arrays with small form factor for AOA [20].

More recently, barometer-aided RSS floor localization has been considered [2, 21, $22,23]$. Barometer-aided RSS methods are attractive due to the complimentary nature of RSS observables and barometer output. Nevertheless, to the best of author's knowledge, current state-of-the-art fusion algorithms based on BA and RSS are rather heuristic and a theoretical framework for BA and RSS fusion, which this thesis aims to provide, does not exist.

\subsection{Objectives and Contributions}

Given the shortcomings involving implementation of various floor localization methods, this dissertation presents a theoretical framework for fusing BA and Wi-Fi RSS readings that can be applied without additional infrastruture for accurate floor localization. With regard to the limitations outlined in the previous section, the objectives of this dissertation can be summarized as follows:

1. Development of a smartphone compatible floor localization algorithm that does not require installation of additional infrastructure and can provide floor localization accuracy comparable to that of DBA-based method. [Chapter 5]

2. Estimation and compensation of drift errors due to local changes in the air pressure observed in BA. [Chapter 3, Chapter 5]

3. Development of a theoretical framework for fusing BA and Wi-Fi RSS. [Chapter 5]

4. Evaluating the robustness and accuracy of the proposed sensor fusion framework along with BA-only, DBA-only, and RSS-only methods for floor localization under different conditions. [Chapter 3, Chapter 4, Chapter 5] 
5. Performance analysis of the proposed method in comparison with BA-only, DBAonly, and RSS-only methods for floor localization over extensive measurements. [Chapter 5]

The major contributions of this dissertation are as follows:

1. Use of Allan Deviation (ADEV) over extensive measurements to characterize underlying random processes (for four state-of-the-art barometers) that influence the BA-based height estimation errors. To the best of author's knowledge, this is the first time use of ADEV is made to characterize barometer errors. [Chapter 3]

2. Development of a tractable mathematical model that adequately characterizes the random process in barometer measurement, and later the model is used to estimate, track, and mitigate barometer measurement errors through sensor fusion. [Chapter 3, Chapter 5]

3. Approximation of Wi-Fi RSS (from plurality APs) distribution in indoor environments with a log-normal distribution. [Chapter 4]

4. Formulation of a Monte Carlo Bayesian Inference (MCBI) framework for floor localization using Wi-Fi RSS. [Chapter 4]

5. Development of a sensor fusion framework for floor localization fusing (combining) both BA and Wi-Fi RSS readings. [Chapter 5]

6. Publication/Submission of scientific papers based on the research work in this dissertation. The list of papers is as follows:

- F. Haque, V. Dehghanian and A. O. Fapojuwo, "Sensor Fusion for Floor Detection," in The 8th IEEE Annual Information Technology, Electronics and Mobile Communication Conference, Vancouver, 2017.

- F. Haque, V. Dehghanian, A. O. Fapojuwo, J. Nielsen, "Wi-Fi RSS and MEMS Barometer Sensor Fusion Framework for Floor Localization" (Accepted for publication in IEEE Sensors Journal). 


\subsection{Dissertation Outline}

This dissertation contains six chapters and three appendices. The remaining chapters are organized as follows:

Chapter 2 provides a literature review on recent RSS-based and barometric pressure (BP)-based floor localization methods. The chapter also includes brief details about the methods that are relevant to floor localization and methods that considered both RSS and BP for floor localization. Benefits and limitations of implementing different methods are also specified.

Chapter 3 develops a MEMS barometer measurement model based on Allan deviation analysis, and the model is later used in the proposed sensor fusion framework. The chapter also presents a framework for BP-based floor localization and studies implementation of BA and DBA for floor localization. The performance analysis of BA and DBA in floor localization over extensive measurements are provided in this chapter.

Chapter 4 studies RSS distribution in indoor environment and it is shown that Wi-Fi RSS from plurality of APs can be approximated with log-normal distribution. This chapter also briefly describes well-known RSS-based floor localization methods and formulates an RSS-based MCBI framework for floor localization. The proposed method is tested against two traditional RSS-based floor localization methods and the results are analyzed in this chapter.

Chapter 5 focuses on the proposed sensor fusion framework, where a Kalman Filter $(\mathrm{KF})$ is utilized to fuse (combine) both Wi-Fi RSS and pressure readings for enhanced floor localization. The implementation steps of the proposed algorithm are briefly described in this chapter. Afterwards, performance of the proposed sensor fusion algorithm is analyzed in comparison with BA-only, DBA-only, and RSS-only methods.

Chapter 6 concludes the dissertation and provides recommendations for future work. Lastly, the appendices detail information relevant to the dissertation. 


\section{Chapter 2: Literature Review}

\subsection{Chapter Focus}

This chapter briefly reviews recent and noteworthy received signal strength (RSS)based and barometric pressure (BP)-based methods that have been developed for floor localization or are relevant to floor localization. In Section 2.2, previous works that involved RSS-based localization are considered, and Section 2.3 summarizes the significant research that used BP for height estimation or floor localization. Section 2.4 discusses the studies that exploited both RSS and BP for floor localization.

\subsection{Prior Research Using RSS}

Nowadays, a Wireless Local Area Network (WLAN) is deployed in almost all indoor environments, such as shopping malls, museums, educational institution, apartment buildings, office spaces, airports and so on. As a result, a large number of Wi-Fi access points (APs) can be seen inside each building and RSS from those APs can be readily utilized for indoor positioning without any additional infrastructure. The two well-known approaches for RSS-based positioning are: 1) fingerprinting, and 2) RSS-based multilateration techniques. In traditional fingerprint matching approach, a list is made with RSS values from the APs at a known position, media access control (MAC) identification numbers (IDs) of those APs, and the coordinate of that reference position. The list is termed as 'fingerprint'. Subsequently, a database is generated that contains many such fingerprints from a set of selected reference positions and the database is termed as radio map. When a user's smartphone scans the Wi-Fi network, the measured RSS values from the scan will be compared to the pre-stored values in the database and, based on that, the system will estimate user position. Particle filtering is one the most popular signal processing methods used in fingerprinting [24]. The set of steps involved in generating a fingerprint database is commonly known as offline or training phase, and the set of steps involved in locating a user is referred to as online phase.

RSS-based multilateration is the other popular RSS-based approach that utilizes the well-known pathloss model for localization [25]. Using RSS values from APs, the model 
estimates distances between a user and the APs. After that the estimated distances are used by some positioning methods such as trilateration (where distances between the user and 3 APs are determined) to locate the user. Traditionally, in this approach, the locations or the coordinates of APs are assumed to be known.

In this section, RSS-based research pertinent to floor localization is classified into three categories. The first category includes studies that utilized larger grid sizes, i.e. fewer areas to collect fingerprints, to reduce the size of the fingerprint database. Selection of larger grid sizes for fingerprint collection results in smaller storage space (memory) on the mobile device. As well, the signal processing in the online phase is faster than that of traditional fingerprinting approach. The second category considers studies that were done either to remove the offline phase from fingerprinting method or to introduce dynamic fingerprinting to incorporate any changes in the fingerprints without manual recalibration. Lastly, the studies that were performed to improve RSS-based positioning by incorporating additional methods or information from various sensors, are classified under the third category.

Under the first category, [20] pointed out that an intensive calibration is required to ensure the quality of RSS-based positioning system. Also, the traditional fingerprinting method will take complete recalibration to accommodate any changes in the Wi-Fi infrastructure. To overcome those two disadvantages [20] designed two models, namely 1) Nearest Floor algorithm, 2) Group Variance algorithm. Nearest Floor algorithm uses a shortened fingerprint database that only contains the MAC IDs of APs, their corresponding floor locations, and the maximum RSS obtained from the APs during the offline phase. To make the fingerprint dynamic, when a new maximum RSS is obtained from an AP during the online phase, the pre-stored maximum RSS against that AP is replaced with the new maximum. Also, information about added or removed APs can also be incorporated in the database. During the online phase, the collected RSS values from the APs are compared with the maximum values stored in the database. Based on that comparison, closest $k$ APs to the user are selected, where $k$ is a number defined by the network administrator. The floor that is associated with those $k$ APs maximum number of times is selected as output of the algorithm. The second proposed model, i.e. Group Variance algorithm, considers 
three statistical parameters: variance of the Wi-Fi RSS readings, range of the Wi-Fi RSS readings, and availability of APs. During the online phase of the Group Variance algorithm, the visible APs from Wi-Fi network scans at a location are paired into groups according to their associated floors. Subsequently, the weighted values of the three parameters corresponding to each group are determined and later added, and the floor with the maximum score is selected as the output of Group Variance algorithm. In the performed experiment in [20], the Nearest Floor algorithm and the Group Variance algorithm achieved $86 \%$ and $72 \%$ floor localization accuracy, respectively, where floor localization accuracy is the ratio of correct to total floor estimates. The difference of the performance was attributed to the construction materials of the buildings. Moreover, [20] stated that Group Variance algorithm outperformed Nearest Floor algorithm in an environment where strong signals were not available, e.g. washrooms, rooms far from APs etc.

[26] suggested a floor localization algorithm based on Bayesian posterior probability and a fingerprint database that occupies less space than the traditional fingerprint database. Rather than taking all APs into account to develop fingerprints, [26] considered the APs which were accessible from at least two floors. Any APs from which the signals were available from only one floor were discarded from the fingerprints. As a result, the new database occupied less space. Nevertheless, the procedure of determining APs that are accessible from multiple floors is labor intensive. During the online phase, the probabilities of observing scanned APs on a specific floor were found. Based on RSS measurements, specific weights were given to the probabilities. Later, corresponding to each floor, the summation of the probabilities was found and the floor with maximum summation was selected as the correct floor. To evaluate the performance, the proposed algorithm was compared with the Nearest Floor algorithm [20]. For the performed experiments in [26], the proposed algorithm achieved $80 \%$ floor localization accuracy on average; whereas, on average the Nearest Floor algorithm obtained 65\% floor localization accuracy.

In [27], an outlier filtering process was presented to remove any large sudden changes in RSS values. For outlier filtering process, the collected RSS values from offline phase were arranged in ascending order to determine 1 st and 3rd quartiles. Using the 1 st and 3rd quartiles, two boundary limits were set, and any RSS values less than the lower limit and 
higher than the upper limit were removed. In addition to the filtering process, standard deviation (SD) of RSS values from all visible APs were exploited to select the most stable APs for the detection process instead of using all APs. The APs that had RSS values with lower SD were assigned as the stable APs, which means RSS from those APs were less affected by multipath interference and, as a result, there were less variations in the RSS values. The APs were sorted in an ascending manner based on their SD values and the top $N$ number APs were selected for the database. During online phase, popular fingerprint matching method $k$-nearest neighbor (KNN) was applied for locating user. KNN first calculates the Euclidean distance (ED) between the collected RSS values on current location and the pre-stored RSS values at certain reference points with known coordinates. $\mathrm{ED}$ is a rough measure of the distance between user and pre-stored known locations. The algorithm selects $k$ number of reference points that give the smallest ED. An average of coordinates of the $k$ reference points gives the user's location.

[28] considered generating offline fingerprints with top $N$-APs from which the strongest signals were received. During the localization phase, the system scanned the network and found top $N$-APs that were transmitting the strongest signals. Subsequently, by comparing the order of the APs (e.g. AP transmitting the strongest signal had order/position 1 on the list) on the offline fingerprints with that of APs observed during online scan, a score was given to each offline fingerprint. The floor associated with the fingerprint with the maximum score was estimated as the smartphone floor location. The proposed method in [28] showed to have 95\% floor localization accuracy when top 11-APs were considered.

Under the second category of the classification, [29] proposed a new floor localization algorithm called sum-RSS floor algorithm, which does not require offline calibration. To replace the need of offline calibration, 2-4 reference nodes (RNs), i.e. wireless transceivers, were installed on each floor. During the online phase, data was collected over 100 network scans to calculate and add average RSS values transmitted by RNs installed on a specific floor. The floor associated with the maximum average RSS was selected as the output of the algorithm. Performance of sum-RSS floor algorithm was compared with that of Nearest Floor algorithm and Group Variance algorithm [20] and it 
was shown that sum-RSS floor algorithm outperformed the other two techniques. Using two and four RNs on each floor in a three-storey building, sum-RSS floor algorithm achieved floor localization accuracy of $91.33 \%$ and $98.67 \%$, respectively. In later work $[30,31,32]$ introduced the use of statistical properties (confidence interval) as a part of previously suggested algorithm in [29]. After determining sum-RSS per floor, a 95\% confidence interval of each sum-RSS was calculated. The floor with the highest confidence interval value indicated the user's floor. Moreover, in [31], rather than using RSS from all reference nodes, $50 \%$ of the RNs on each floor were selected from which the strongest signal strengths were received in each scan of the network. In addition to that, in [32], the experiments were performed to estimate user's floor with fault-free and faulty RNs. The RNs that worked properly during online phase was termed as fault-free RNs; whereas the RNs that stopped working or failed during online phase were termed as faulty-RNs. In scenarios with fault-free and faulty RNs, the floor localization accuracy was $100 \%$ and $95.1 \%$, respectively. Note that the algorithms proposed in $[29,30,31,32]$ require new infrastructure (i.e. installation of RNs) and network scans over a longer period to improve floor localization accuracy. The high accuracy in [29, 30, 31, 32] can also be attributed to the fact that the reference nodes were distributed on each floor such that the maximum and uniform coverage was obtained from the RNs on each floor. Subsequently, the uniform arrangement of RNs made it possible to place the two or four RNs in a way so that the RNs approximately had the similar placement on each floor, which is not always the case in real world. For a specific area of a floor, non-uniform placements of the RNs can result in receiving the strongest signals from the RNs placed on adjacent floors, which will impact the accuracy of the algorithms presented in [29, 30, 31, 32]. Furthermore, the detection process for a single test point was based on 20-100 samples, which required a long time (1 minute for 20 samples [32]) for estimating user floor location; and so, this is not favorable in real-time applications. Along with that, the number of selected test points (15 points in $[31,32]$ and 50 points in $[29,30,31,32])$ on each floor was not sufficient to reach a general conclusion.

[33] described a trajectory learning mechanism that uses crowdsourcing for indoor positioning when fingerprints of RSS and floor map are not available. Also, feature extraction algorithm was included in [33] to reduce the size of the fingerprint. Linear 
discriminant analysis (LDA) and principal component analysis (PCA) are the two feature extraction techniques that were used in a combination in [33]. From the scanned RSS measurements, PCA and LDA remove the elements that do not add any value to the detection process, such as empty or meaningless RSS values due to APs from which no signals are received at a location. PCA uses only the RSS measurements to extract the essential data; whereas, LDA uses labeled information, i.e. RSS measurements associated with a floor, a landmark, or a position. During the offline phase, [33] used primary RSS measurements vector (observant vector) and a transformed vector with reduced dimensionality of that observant vector to calculate a transformation matrix. Solving for transformation matrix included an optimization problem and it was solved using LDA and PCA. During the online phase, a transformed vector was found for each scan using the transformation matrix found in the training phase. Subsequently, KNN algorithm [27] was applied to the transformed vector (i.e. reduced observant vector) to estimate user floor location.The proposed algorithm in [33] had an error of 2.2 meters on average, which lacks the required accuracy for floor localization. Authors in [34] suggested a dynamic adjustment in the RSS-fingerprint to remove the effect of APs' transmitted power variation. The transmission power can vary automatically to maintain the transmission quality in the network. As an example, it was shown that during a performed experiment, the transmission power of an AP decreased $3 \mathrm{dBm}$ per hour over a 5 hours measurement period, before it started re-transmitting with the expected power. To cancel out the effect of power variation, [34] measured the RSS among APs along with traditional RSS-fingerprints during the offline phase. During the online phase, when variation in RSS among APs was detected, the information was incorporated into the floor localization algorithm. [34] showed that dynamic adjustment algorithm helped to keep the regular position accuracy stable under the transmitted power variation of the APs.

As stated earlier, the third category incorporates information from additional sensors or other methods to improve RSS-based positioning. [3] proposed cooperative information sharing between mobile nodes and integration of inertial measurement units (INS) with RSS to improve indoor positioning. In cooperative sharing, the neighboring mobile nodes share their RSS observables from APs along with range measurements between the nodes, where the range is determined using RSS-based multilateration technique. Incorporating 
cooperative sharing with RSS can significantly improve indoor positioning and it makes it possible to design a localization system with a small number of reference nodes even in large-scale environment. Additionally, [3] used measurements from INS as prior information to further improve the accuracy of location estimation. In [25], authors utilized M-estimator, which is a generalization of maximum likelihood (ML) estimation, to make four existing approaches based on RSS more robust. The four approaches were: weighted centroid localization (WCL), linear and non-linear trilateration, and deconvolution-based approach. In WCL approach, the location of smartphone is estimated as the weighted average of the distances between smartphone and APs. Non-linear trilateration approach considers minimizing a square function based on RSS values and distances between smartphone and APs. Whereas, to estimate smartphone location, linear trilateration technique uses a least square method to solve for linear equations based on distances between APs and smartphone as well as distances between two APs. The deconvolutionbased approach uses methods such as least squares, weighted least squares, mean square error to estimate smartphone location based on RSS pathloss model. [25] showed that using regular WCL, NLT, and deconvolution-based approach the floor localization accuracies were $85 \%, 73.3 \%$, and $66.5 \%$, respectively. When the robust version of WCL, NLT, and deconvolution-based approach was used, the floor localization accuracies increased to $85.8 \%, 75.6 \%$, and $68.3 \%$, respectively.

[35] proposed a $\mathrm{Wi}-\mathrm{Fi}$ aided pedestrian dead reckoning algorithm for indoor localization. The algorithm in [35] used inertial measurement unit (IMUs) to determine the maximum and the minimum acceleration over a sliding window and compare the difference of those values to a threshold to identify pedestrian's activities such as standing, walking, or taking stairs. In addition, [35] used the change in RSS values from the APs to determine any movement between floors. [35] showed that $70 \%$ of the distance errors for the proposed algorithm were less than 1 meter. Note that the results in [35] were not based on extensive measurements, and [35] assumed access to some information, e.g. height of stairs, slant angle of stairway, which are not conveniently available when considering practical scenarios. In another study, [36] combined RSS fingerprint and magnetic field fingerprints to improve the accuracy of indoor positioning. Stairs and elevators were defined as landmarks and accelerometer was used to detect when someone was in vicinity of these 
landmarks. [36] showed that the use of landmarks along with the RSS and magnetic field fingerprints improved localization accuracy.

[37] introduced a sequential approach to RSS-based positioning that uses a Bayesian metric to find the likelihood of a particular path. During the offline phase, fingerprints were collected at some certain reference points, and during online phase, KNN algorithm was used to determine the probability of a user being on a particular location conditioned on a time series of RSS observations. [37] showed that increasing the length of the series of RSS observation increased the probability of correct path estimation and reduced the average distance error. Average distance error represented the distance between true location of the user and the estimated location by the system. The experimental result in [37] showed a probability of correct path estimation of larger than $97 \%$ and average distance error of less than 0.1 meter. The average distance error determined in [37] using RSS is unusual in RSSbased positioning techniques. In [38], it was shown that the level of accuracy that can be achieved using methods based on signal patterns along the trajectory (known as Temporal patterns) is less than 2 or 1.8 (based on which methods are used) meters. The rationale behind the high correct location estimation accuracy in [37] can be that the experiment was conducted only on a hallway of a single floor and only 18 locations were selected for measurements during the online phase. The locations were also really close to each other (1 meter apart) and 10 online measurements were drawn from each location which mitigated the multipath effect. Moreover, multi-story building introduces some challenges in location estimation, which was not considered in [37]. Also, [38] stated that the positioning becomes difficult if the user moves faster or in different directions; these scenarios were not exploited in [37]. Moreover, the conclusion about the accuracy was based on only 100 realizations, which is not sufficient.

\subsection{Prior Research Using BP}

The inclusion of BP sensors (i.e. barometers) in modern gadgets (smartphone, smartwatches etc.) has augmented the interest of using barometers in the field of indoor positioning. Pressure measurements from barometer can be converted to equivalent height, which subsequently is used for floor localization. Many recent studies have been done using pressure sensors for height measurements and floor localization. Researchers either 
used measurements from a single pressure sensor or from two pressure sensors for the conducted studies. Use of a single barometer for height measurements is known as barometric altimetry (BA). Whereas, differential barometric altimetry (DBA) uses a barometer specifically as reference and another barometer as a roaming node to measure the relative height between those two sensors.

In [39], barometer was deployed for BA and it was shown that pressure drift is observed during data collection due to changes in the local environment, e.g. influence of wind, air-conditioning system etc. [39] suggested using height observation from GPS or returning to a point (with known height) inside a building to calibrate for pressure drift. A linear characteristic curve was derived to determine relative change in height for a given change in pressure. Based on a 6 minutes measurement set, it was shown that $91 \%$ of the time floor transitions could be detected with an error of less than $1 \mathrm{~m}$ and it was concluded that the error could change depending on the time of the day the measurements were taken.

[40] worked with different barometer models to verify whether those sensors could be used to accurately detect changes in heights and transportation modes (e.g. elevator, escalator or stairs). Three parameters, i.e. difference in BP measurements, the rate of change of BP, and transition time to move between floors, were considered to determine exact number of floor changes and the transportation mode used by the smartphone user. Transportation mode was detected with $100 \%$ accuracy when both rate of change of BP and transition time were considered. To accurately detect the transportation mode, [40] assumed that the required time to change a floor was different for different transportation modes. Apart from detecting transportation modes, [40] used BA to determine floor changes and it showed $100 \%$ localization accuracy when the user moved just one floor up or down. For other scenarios, the accuracy of correct detection of exact number of floor movement dropped to $60 \%$ because the time required to move between floors was same for different transportation modes. In comparison, [40] showed that accelerometers embedded inside smartphones achieved $40 \%$ accuracy in determining mode of transportation and 50\% accuracy in detecting a floor change. Also, it was mentioned that barometers consume 3-23\% less power than accelerometers do. Moreover, [40] demonstrated that the value of absolute pressure measured by barometers embedded inside 
smartphones varies throughout the day because of changes in diurnal temperature and weather. In addition, [40] showed that the pressure readings measured at a fixed location varies over different models of smart-phones. Even the same models of smart-phone can provide different measurement readings while measuring BP from the same place. For example, during pressure measurements with two Googly Galaxy Nexus, a pressure difference of $1.2 \mathrm{hPa}$, which is equivalent to height difference of $10 \mathrm{~m}$, was observed in [40].

[15] illustrated that environmental factors (closing or opening doors in a room, ventilation system, and weather conditions) greatly impacts the pressure readings of a single barometer. Fortunately, differential pressure sensing process in DBA compensates that effect as both reference and rover barometers are influenced by similar change in the environment. As such, [15] suggested the use of DBA to accurately measure height difference (estimation within centimeter range) for medical application, such as determining body orientation of a patient who needs intensive care. To estimate the height, [15] used a barometric equation that considers density of air (which depends on temperature and humidity) and showed that the pressure measurements were not significantly influenced by humidity and temperature. A maximum error of $17 \mathrm{~cm}$ was seen for a $20^{\circ} \mathrm{C}$ variation in the temperature; whereas, only $1 \mathrm{~cm}$ of maximum error was observed for 10-90\% variation in the humidity. Additionally, [15] suggested the use of moving average filter to smooth the measured raw readings and to give a better precision. However, moving average filter adds delay to the localization phase and it was shown that a moving average filter with order 60 (i.e. averaging over 60 samples) added a lag of 4 seconds. Based on a 35 seconds measurement, [15] demonstrated $4.2 \%$ height estimation error.

In [41], the suggestion of using multiple reference barometers was given. Reference barometers were placed on each floor inside a building to measure real-time pressure readings at each level. Afterwards, the measured pressure readings were uploaded to a server to make a real-time pressure map. Pressure reading from user smartphone was compared with the measurement stored in the pressure map to determine user floor location. Note that offset calibrations were performed for all the barometers involved in 
the measurements. The achieved floor localization accuracy for the conducted measurements in [41] varied from $88 \%$ to $99 \%$. [41] also demonstrated small improvement in floor localization by taking averages over multiple samples, which averaged out the fluctuations in the measurements. For instance, floor localization accuracy improved from $95.65 \%$ to $96.06 \%$ when 5 seconds time window was considered for decision making instead of 1 second window.

Similar to [41], [42] and [43] considered generating pressure map (i.e. pressure fingerprints) of each floor inside a building. Unlike [41], [42] and [43] used crowdsourcing to form the pressure map. This map was updated in a timely basis and uploaded to cloud server. The measured pressure values by the user smartphones were clustered with a specific time stamp and if changes in pressure was observed on a floor, then all pressure values on other floors were updated as well. The fact that the pressure difference between two floors does not change over time - was considered in map updating process. The pressure map generating and updating process required calibration among smartphones and it was performed when users shared the elevator to a certain floor. For localizing a user, user smartphone was required to download the calibration information and the pressure map from the cloud through an app. Subsequently, the readings from barometer embedded in user smartphone was used by the app to determine user floor location by comparing the real-time measurements with the pressure readings from the pressure map. Both field experiments and simulation were done by [42] and [43] to observe the efficiency of the proposed localization system. The system achieved $98 \%$ floor localization accuracy when calibration among smartphones was possible and $70 \%$ floor localization accuracy when calibration could not be done (e.g. at the beginning of a day, when there are not sufficient users inside a building).

In [44], a self-adapting method was introduced that detected any pressure drift due to environment and made correction to the measurements for estimating user floor location. The proposed method in [44] had two stages: recognition and correction. In the recognition stage, a window containing most recent pressure measurements was considered to determine an average pressure. Subsequently, difference between the average pressure and the current measurement was determined, which was then compared with a threshold to 
decide whether the change in pressure was related to environment or floor transitions. The threshold depended on the maximum and the minimum pressure readings in the selected window. When a pressure change due to environment was detected, the initial measurement after the last floor transition was compared with the most recent reading to calculate the pressure offset, which was used in pressure drift correction. [44] also integrated a beacon system that transmitted pressure readings measured by a reference barometer at a certain interval. Any user terminal (i.e. smartphone) receiving the signal transmitted by the beacon, if needed, would make appropriate changes to the measurements. For the performed measurements, [44] achieved floor localization accuracy between 85\%-95\%.

In [19], the researchers combined DBA and measurements from accelerometer using a complementary filter to estimate height. DBA was used to remove the drift error observed in BA and measurements from the accelerometer were used to mitigate the stochastic error (i.e. high-frequency random noise) in pressure measurements. Low-pass filter and highpass filter were used to reduce the stochastic error in DBA and accelerometer output, respectively. [19] showed that using the proposed method a standard deviation of 0.0679 meter was achieved in the output compared to the original 0.4661 meter. [45] compared the results that were found using DBA and BA to estimate relative height. For using DBA, the offset between the sensors was removed by initially placing both reference and rover barometers at the same height. For the performed experiments in [45], BA and DBA resulted in $41.49 \%$ and $12.76 \%$, respectively, height estimation error. [45] also discussed how at different pressure levels the differential pressure measurements (i.e. the outcome of differencing operation in DBA) could change in a nonlinear way and it was concluded that deviation in the measurements occurred due to dissimilarities in sensors' pressure-response curves. For example, it was shown that a pressure change of 0.06 bar or $60 \mathrm{hPa}$ resulted in a deviation of $160 \mu$ bar or $0.16 \mathrm{hPa}$ in differential pressure measurements, which is equivalent to a height error of 1.35 meters. Based on observation, [45] concluded that a one-time calibration of reference and rover barometers might show some measurement error over time. 


\subsection{Prior Research Using Both RSS and BP}

In recent years, few studies have been conducted where both RSS and BP were used for indoor positioning. Using both techniques simultaneously, the individual drawbacks of both methods are compensated. [2] introduced a hybrid approach that utilized Wi-Fi RSS and BP readings for floor localization. Initially [2] formulated a Bayesian classificationbased floor identification (BCFI) algorithm that generated Wi-Fi RSS fingerprints and then used the fingerprints to determine user floor location based on the probability of being located on a floor. [2] also demonstrated a barometric pressure-based floor identification (BPFI) algorithm that created an altitude-based floor map from the initial BP measurement and initial floor number given as an input by the user. Self-calibration in BPFI was done based on detecting the magnitude of change in pressure over time. If the pressure changed more than a specified threshold after a certain identification period (15 seconds), then a change in floor level was detected. Otherwise, a slow change in pressure was detected and the floor map was updated based on the last measurement. BPFI could not detect floor transition when a user changed the floor at a very slow speed; and there was a delay in detection through BPFI because of the identification period. The suggested hybrid approach combined BPFI with BCFI, which removed the necessity of providing the initial floor number as a manual input and could detect change in floors even when the user was moving at slow speed. In hybrid approach, BPFI was initialized and calibrated only when a floor identification from BCFI was obtained with high probability. It was not possible to identify floors with high probability using BCFI in an area with hollow interiors. [2] showed $96.1 \%$ floor localization accuracy using hybrid approach over a 20 minutes measurement set.

In [21], fingerprints comprising RSS and BP readings were stored in a server through crowdsourcing as a training dataset. The pressure measurements among multiple smartphones from the training dataset were compared to a pre-determined value to identify smartphone floor locations and consequently corresponding collected RSS fingerprints were assigned to specific floors. Later, in localization phase, RSS measured by user smartphone were sent to the server and compared with the training dataset to determine user floor location. The method mentioned in [21] achieved more than $90 \%$ floor 
localization accuracy; however, it is computationally complex because it required integration of crowdsourcing, comparison of RSS fingerprints, and calibration among barometers of different smarphones.

In [23], researchers used RSS-based radio propagation model with ML estimation to identify correct floor location. The ML estimation was based on maximizing the RSS measurement obtained from access points on each floor. Further, barometer was combined with the RSS-based approach so that the system could perform well even when the signal strength was poor or not enough APs were visible through Wi-Fi scans. The barometer measured the pressure, from which relative altitude changes and consequently vertical velocity was found through differentiation. Afterwards, the vertical velocity was compared with a threshold to detect any floor transitions. If it was detected that the user was using stairs or elevator, the algorithm would show the last detected floor using RSS as output until user reached the new floor. Over a minute lasting measurement, the proposed algorithm achieved more than 99\% floor localization accuracy. The suggested method in [23] used ML framework, which is prone to singularities for problems involving Gaussian mixtures such as Wi-Fi RSS observables inside buildings. Also, performance analysis over extensive measurements was not carried out.

[46] demonstrated a technique that utilized both RSS-based fingerprint matching method and barometric pressure for 3-D localization. In the experiment, the technique used two filter windows in time domain for processing height measurements based on barometer readings. The first window considered the current samples for measuring average of measured heights. The second window used past measurement samples to calculate an average height. Subsequently, the difference between the two average heights was compared with a threshold to detect floor transitions. When a floor transition was detected, the total change in floor numbers was found assuming building height information was available. The RSS measurements were used to determine user's initial floor location and to find the $(\mathrm{x}, \mathrm{y})$ coordinates. Assuming all the floors of a building have the same height, the researchers detected the floor for the experiment with 0 false floor detection. The limitation of the technique used in [46] is that the technique falsely assumed same height difference between any two floors of a building. Also, the technique did not consider the 
scenarios where a user may make slow or fast transitions between floors. The value of involved parameters, such as length of the filter windows, time difference between the two windows, threshold etc., can vary depending on buildings.

[47] considered a crowdsourcing algorithm that used inertial measurement units (IMUs), RSS from Wi-Fi access points and BP for indoor positioning. Information from the users' walking trajectory was collected by a server that included readings from IMUs and barometer, and Wi-Fi RSS at each step of the user. Afterwards, the server compared and matched the information obtained from the users and then clustered that information to create a floor plan. In the algorithm, no previous knowledge about the environment was needed and the floor map and radio map were auto updated through crowdsourcing. However, periodically the users were required to give their locations as an input to the algorithm. Moreover, the proposed process in [47] required the server to continuously do complicated computation and the algorithm did not consider an open space building structure. [47] showed an accuracy of 1 3 meters in positioning along with $98 \%$ average accuracy in floor transition detection.

The proposed method in [48] had two stages for floor localization with. In the first stage, RSS-fingerprint based method, such as weighted KNN algorithm, was utilized to identify the floor. The suggested weighted KNN was similar to ordinary KNN [27] algorithm, except a weight value was given to the obtained RSS based on their values. In the second stage, [48] measured change in pressure after every certain number of steps to detect any movement between floors. Change in pressure was measured by using barometer and number of steps were measured using accelerometer. Based on a single experiment, [48] showed an accuracy of $97 \%$ in detecting floor changes and $99 \%$ in identifying the proper floor. Note that during the measurement, only 2 floors with 301 reference points were selected for the training phase.

[4] proposed a method using Bluetooth Low Energy (BLE) RSS and BA for floor localization. The method required installation of BLE beacons and a server capable of continuous real-time information processing. According to the method, when access to BLE beacons with known coordinates was available, the measured pressure by a smartphone was stored in the server as reference atmosphere pressure, which was updated 
periodically when the same smartphone had access to the beacons again. During localization, the measured BP was compared with the reference atmosphere reference corresponding to each floor to determine smartphone floor location. Moreover, calibration among smartphones was performed when multiple smartphones were in vicinity of the same BLE beacon and measurement from one of the smartphones was considered as reference for calibration. The pressure difference between adjacent floors was determined when calibrated smartphones detected installed BLE beacons on two different floors. [4] showed a floor localization accuracy of $91 \%$ over a measurement of 23 minutes.

\subsection{Relationship between the Thesis and Previous Works}

The prior works that considered both RSS and BP readings for floor localization are rather heuristic and failed to formulate a theoretical framework for combining both readings. As well, performance of the previously proposed methods was analyzed over short time periods and/or based on limited measurements. In addition, prior works were computationally complex due to integration of various factors, such as crowdsourcing, barometer calibrations, RSS fingerprints comparison etc. This thesis proposes a sensor fusion algorithm (see Chapter 5 for details) that not only provides a theoretical framework for Wi-Fi RSS and BP (more specifically BA) fusion, but also has high floor localization accuracy under different experimental conditions. The proposed method uses a Kalman filter $(\mathrm{KF})$ to predict the drift (i.e. the slowly varying mean) in BA using a prediction model introduced in Chapter 3. Correction to the prediction is performed when access to Wi-Fi RSS is available using an RSS-based Monte Carlo Bayesian Inference (MCBI) framework formulated in Chapter 4. As will be shown, both RSS and BA-based techniques for floor localization will be used as an integrated part of the sensor fusion framework. Unlike prior fusion methods, the performance analysis of the proposed method (provided in Chapter 5)

along with BA-only, DBA-only, and RSS-only methods (detailed in Chapter 3 and Chapter 4) was performed over extensive measurements. 


\section{Chapter 3: Floor Localization Using Barometric Pressure}

\subsection{Introduction}

It is well known that atmospheric pressure varies with altitude. Barometric pressure (BP) sensors have long been used by hikers and climbers, and Micro-electro-mechanicalsystems (MEMS) barometers, i.e. BP sensors, are highly integrated into positioning systems for altitude measurements. The integration of MEMS BP sensor in state-of-the-art handheld devices, such as smartphones (e.g., Samsung S4/S8/note 2/3/4/8, iPhone 6/7/8/X, Xiaomi 2/3/4/5, Google Pixel 2, etc.) and smartwatches, has prompted a surge in its use in indoor positioning system (IPS). One simplified model for converting atmospheric pressure measurements to height is given by [2]:

$$
h_{m}=44330 \times\left(1-\frac{\mathcal{P}}{\mathcal{P}_{o}}\right)^{\frac{1}{5.255}}
$$

where $h_{m}$ is the calculated height with reference to sea level, $\mathcal{P}$ is the BP measured by the sensor, and $\mathcal{P}_{o}$ is the pressure at sea level, which is $1013.25 \mathrm{hPa}$ or $101325 \mathrm{~Pa}$. Up until now researchers have studied the use of single BP sensor for barometric altimetry (BA) and multiple BP sensors for differential barometric altimetry (DBA) to detect smartphone user floor location. Floor localization system using BA and DBA both have their advantages and disadvantages that need to be considered before implementing the system. BA can be readily implemented with the smartphones of current vintage; however, BA is prone to drift errors due to local changes in the air pressure. Whereas, DBA can provide with required floor localization accuracy, but additional infrastructures need to be installed.

The objective of this chapter is to briefly discuss use of barometer in floor localization, and compare performance of BA and DBA in floor localizatoin. The rest of the chapter is organized as follows. In Section 3.2, MEMS barometer measurement model is developed using Allan deviation (ADEV). Section 3.3 describes a maximum a posteriori (MAP) classifier based on minimum probability of error criteria for floor localization using BP. Section 3.4 details the equipment and environments selected for conducting 
measurements. Descriptions and results of floor localization using BA and DBA are given in Section 3.5 and Section 3.6, respectively. Finally, Section 3.7 summarizes this chapter.

\subsection{MEMS Barometer Measurement Model}

While (3.1) provides a direct mapping between the measured pressure $\mathcal{P}$ and height $h_{m}$, local air pressure is significantly influenced by unknown disturbances such as approaching high/low pressure weather systems, air-conditioning, wind, temperature, etc., resulting in large height estimation errors. For accurate floor localization using MEMS BP sensors, the measurement errors must be modeled and accounted for.

In [11] BP-based height estimation errors are categorized into three groups: bias error, drift error, and drift-rate. [49] proposed a different error model, where the drift error is modeled as a first order Random Walk (RW). Others, e.g. [2], simply treat BP-based height estimation error as a single unknown error term which needs to be estimated and removed from each measurement snapshot. While most of the previously proposed error models may be valid for certain datasets, to the best of author's knowledge those conditions are not properly discussed and understood. In this section, the underlying random processes that influence the BP-based height estimation errors are characterized.

Let $\Delta_{h}$ denote the height error due to a plurality of factors such as approaching high/low pressure systems, air conditioning, factory calibration (or the lack of), etc. such that

$$
\tilde{h}=h+\Delta_{h}
$$

where $\tilde{h}$ is the measured height, relative to a local reference point, e.g. first floor of a building calculated from a MEMS BP sensor output, and $h$ denotes the correct height relative to the same reference point (e.g. from surveying). The variables $\Delta_{h}, h$ and $\tilde{h}$ are time-varying random processes. Figure 3.1 shows $\Delta_{h}$ for two set of measurements made over two separate 115 hours long periods using a BMP180 barometric pressure sensor. During the measurements, the sensor(s) under test (SUT) was kept at a fixed position inside an office building that was subject to climate control pressure changes and data was captured at 1 second intervals. As evident from this Figure 3.1, $\Delta_{h}$ is a non- stationary 


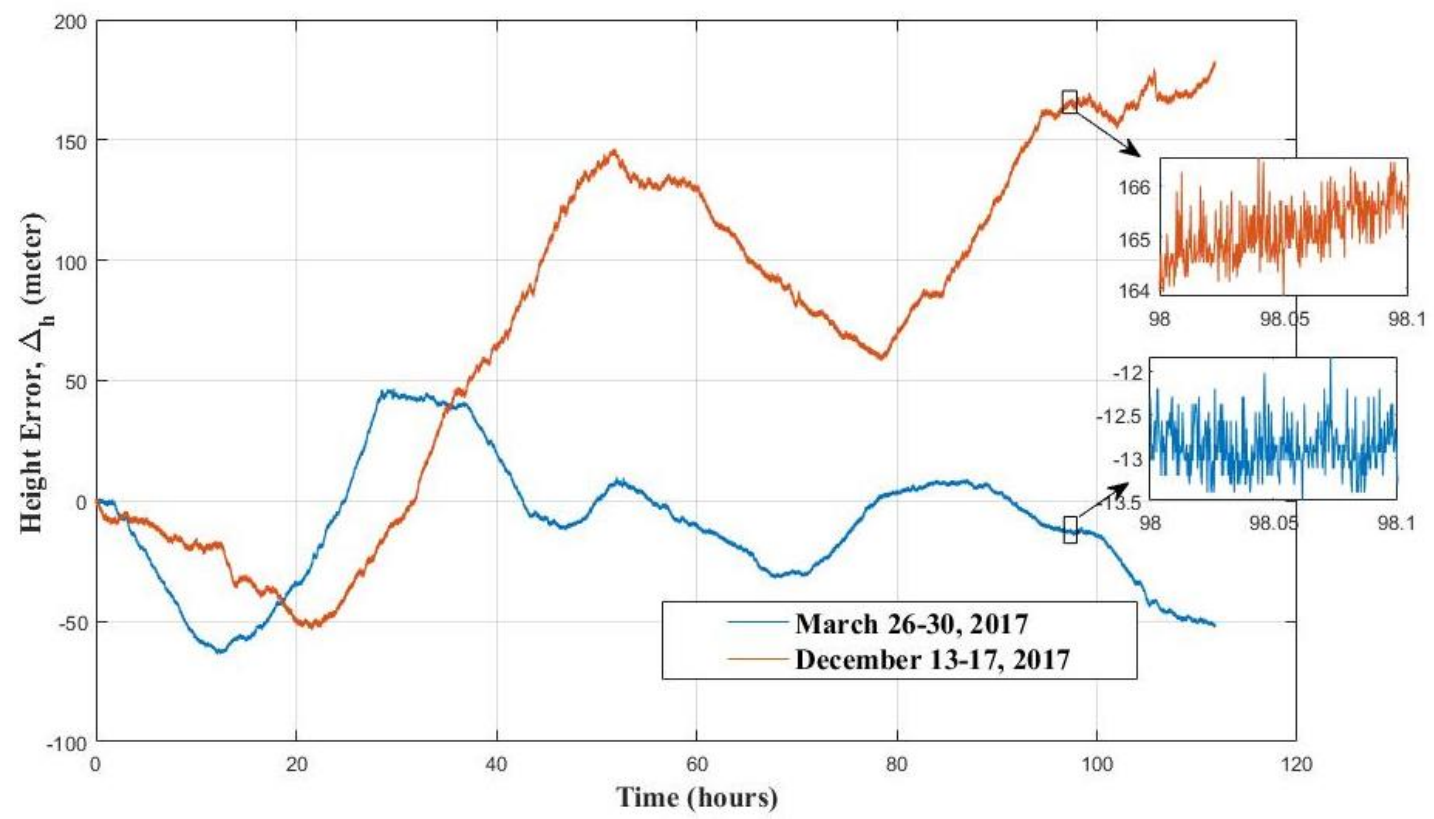

Figure 3.1: Determined height error $\left(\Delta_{h}\right)$ for 115 hours long measurement set

random process comprising a fast varying zero-mean noise and a slowly varying random mean. The slowly varying random mean, hereafter referred to as random bias, is commonly attributed to the approaching weather systems among other influencing factors (e.g. airconditioning) and the zero-mean noise component is generally due to digitization, thermal noise, etc. Note that such characterization of error sources is out of the scope of this work.

Allan deviation is a time-domain analysis technique suitable for characterizing (non) stationary random processes and has been extensively used for characterizing inertial sensor and oscillator errors $[50,51,52]$. In this section of the dissertation, ADEV analysis is used to characterize the non-stationary errors affecting barometer measurements. ADEV and power spectral density (PSD) of a random process are related through an integral equation and it is shown that different random processes appear as slopes of different gradients on the ADEV plot [53]. For instance, White Gaussian Noise (WGN) processes manifests itself as a straight line with a slope of $-1 / 2$ on the an ADEV graph. Also, the flat part of an ADEV graph (slope of zero) is typically investigated for characterising bias instability. While ADEV is a powerful time-domain tool for characterizing sensor errors, it is important to note that unlike inertial sensors and oscillators, where the analysis provides information about the intrinsic random noise processes, dominant sources of error in BP-based altimetry are external and vary significantly over extended periods of several 
hours. As such, our analysis and modeling of BP sensor noise will be limited to those internal/external noise processes that are common across various usage cases, which includes random noise processes that are mainly dominant over short-term i.e. up to several minutes. One realization of ADEV based on non-overlapping clusters is given as [53]

$$
\sigma_{A D E V}\left(\tau_{c}\right)=\sqrt{\frac{1}{2(\mathcal{C}-1)} \sum_{k=1}^{\mathcal{C}-1}\left(\bar{\psi}_{k+1}\left(\tau_{\mathrm{c}}\right)-\bar{\psi}_{k}\left(\tau_{\mathrm{c}}\right)\right)^{2}}
$$

where

$$
\bar{\psi}_{k}\left(\tau_{c}\right)=\frac{1}{N_{c}} \sum_{n=(k-1) N_{c}+1}^{k N_{c}} \tilde{h}[n]
$$

is the $k$-th cluster average, $\tau_{c}$ is the cluster time (averaging time), $\mathcal{C}$ is the total number of clusters, $N_{c}=\tau_{c} F_{S}$ is the number of samples per cluster, $F_{S}$ is the sampling frequency, and $\tilde{h}[n]$ is the measured height, defined in (3.2), during the $n$-th sampling interval.

Four state-of-the-art MEMS barometers (namely BMP180 [54], BMP280 [55], BME280 [56], and MPL3115A2 [57]) that are commonly used in various consumer electronics products and smartphones of current vintage are considered for the measurements. In order to isolate internal/external random variations in atmospheric pressure from those due to a deliberate altitude change, the SUT were placed adjacent to each other inside a typical office building and at the same height. ADEV, from (3.3), was then calculated for each SUT and the results are shown in Figure 3.2. For notational brevity, only the last four letters of SUT product identifications are noted.

From Figure 3.2, two categories of random processes can be identified: 1) the WGN, 2) Colored Noise of $1 / f^{\alpha}$ PSD, with exponent $\alpha \geq 1$. As mentioned before, WGN is manifested as a slope of $-1 / 2$ on the ADEV graph. As can be seen from Figure 3.2, WGN process is the dominant noise process over short observation periods, i.e. cluster time of $\tau_{c}<T_{b}$, where $T_{b}$ denotes the time interval over which the WGN is the dominant noise process. From Figure 3.2, $T_{b}$ ranges from 6 to 15 seconds across the SUTs considered in the measurements. Also, Figure 3.2 shows that one-sample deviation ranges from 10-15 


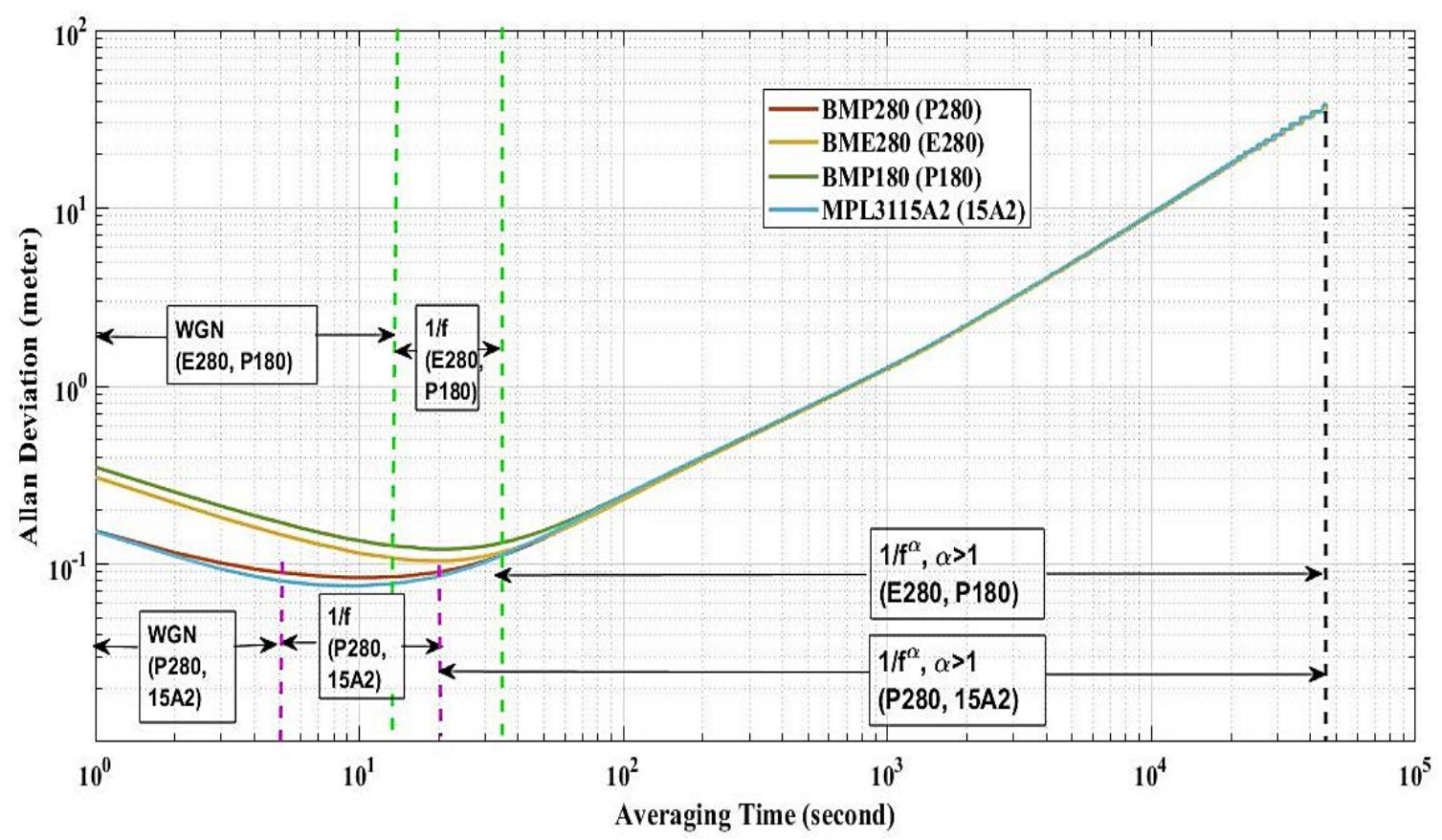

Figure 3.2: Allan deviation plot for MEMS barometer of different models

$\mathrm{cm}$. The one-sample deviation is related to WGN power as the square root of noise power for a single point measurement $[53,58]$.

Investigating the flat part of the ADEV curves in Figure 3.2 reveals that colored noise process of $1 / f$ PSD (also known as bias instability) is the dominant noise process when $\tau_{c} \approx T_{b}$ with deviations ranging from $0.075-0.15 \mathrm{~cm}$. The parts of ADEV with asymptotes of positive slopes are generally related to noise process with PSD of $1 / f^{\alpha}, \forall \alpha>1$, which are dominant for time periods of $\tau_{c}>>T_{b}$ seconds. This part of ADEV curve is common across all sensors as can be seen from Figure 3.2. As such, $1 / f^{\alpha}(\alpha>1)$ can be attributed to external sources of error, e.g. changes in the surrounding atmospheric pressure. To further verify, ADEV curves of DBA (as shown in Figure 3.3) are considered. DBA based on (near-) real-time differencing of measured barometer output and the measured barometric pressure of a reference barometer of known altitude are effective in removing random drift errors caused by the common (external) sources and providing decimeter level accuracy $[10,14,15]$. As a result, any noise terms visible on the ADEV curve are solely due to the sensor's intrinsic noise. From Figure 3.3, only WGN and $1 / f$ noise can be observed. Hence, it is evident that $1 / f^{\alpha}(\alpha>1)$ noise in barometric altimetry is introduced 


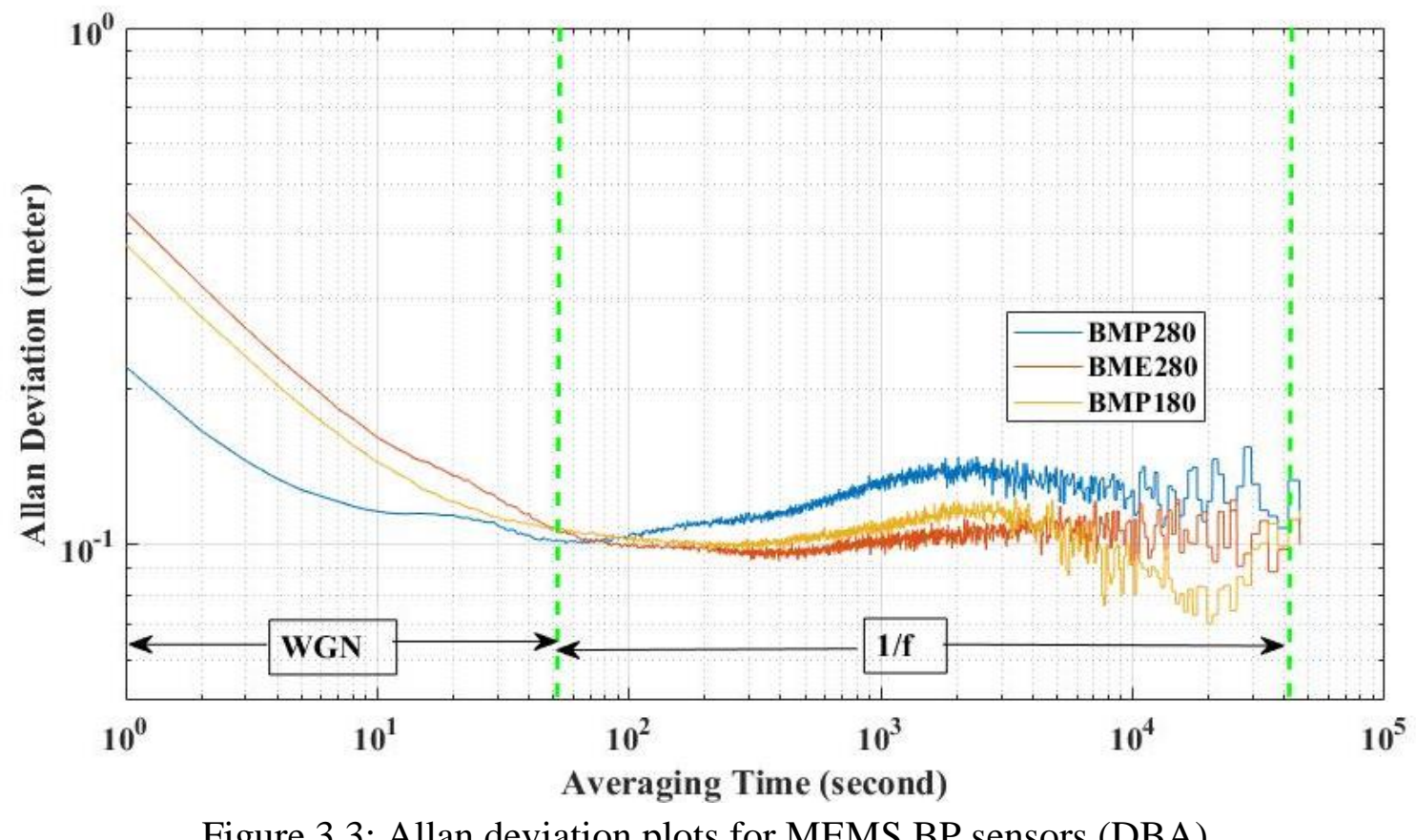

Figure 3.3: Allan deviation plots for MEMS BP sensors (DBA)

by the external sources. Note that the differencing operation in Figure 3.3 increases the $\mathrm{ADEV}$ of $\Delta_{h}$ and the time interval for which WGN and $1 / f$ noise are dominant.

From measurements it was observed that the value of $\alpha$ for $1 / f^{\alpha}(\alpha>1)$ colored noise process varies with test time and geographical location. As shown in Figure 3.4, the magnitude of $\alpha$ (visible through different slopes) varies with usage case. The data to generate the figure were collected in three different weeks inside three different buildings (more details about the buildings are given in Section 3.4.2) on the University of Calgary campus. The varying magnitude of $\alpha$ makes it impractical to use a single definite model for $1 / f^{\alpha}(\alpha>1)$ noise. TABLE 3.1 gives a more detailed summary of the various random noise processes in a MEMS barometer for the SUTs considered in this thesis. As can be seen from TABLE 3.1, the deviation introduced by WGN and $1 / f$ noise and the time interval for which these are the dominant noises in the output of BMP280 and MPL3115A2 are less than that of BMP180 and BME280.

As stated earlier and is evident from Figure 3.2 and TABLE 3.1, WGN process can be used to accurately model BA error for up to several seconds and colored noise processes dominate $\Delta_{h}$ when longer observation periods are needed. There are two major difficulties 


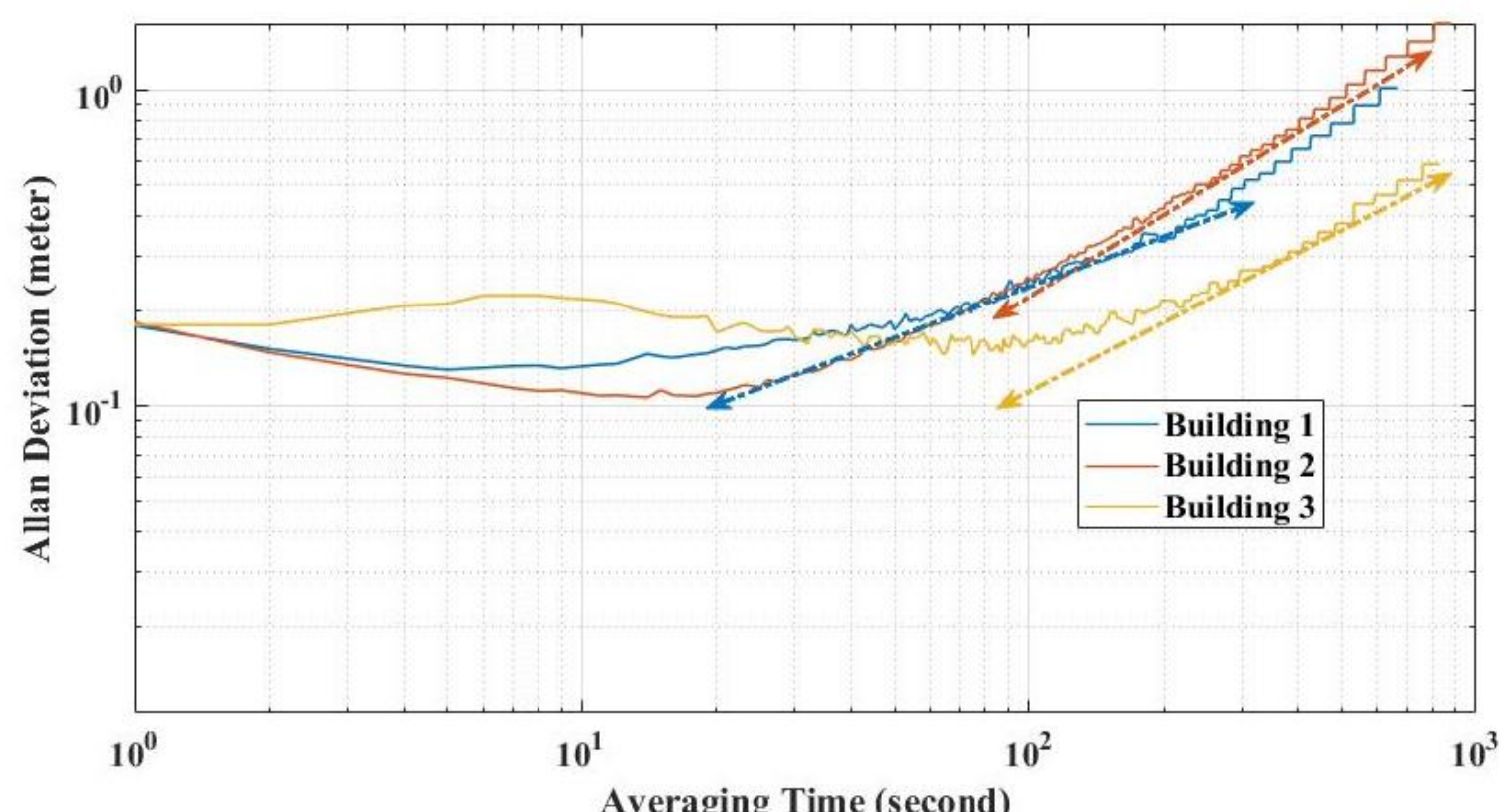

Figure 3.4: Varying magnitude of $\alpha$ for $1 / f^{\alpha}(\alpha>1)$ noise

TABLE 3.1

NOISE CHARACTERISTICS OF THE TESTED BP SENSORS

\begin{tabular}{|c|c|c|c|}
\hline Sensor Model & Dominant Noise & $\boldsymbol{\tau}_{\boldsymbol{c}}(\mathbf{s})$ & $\boldsymbol{\sigma}_{\text {ADEV }}(\mathbf{m})$ \\
\hline \multirow{3}{*}{ BMP280, MPL3115A2 } & WGN & $<6$ & $<0.15$ \\
\cline { 2 - 4 } & $1 / f$ & $6-20$ & $0.075-0.08$ \\
\hline \multirow{3}{*}{ BMP180, BME280 } & $1 / f^{\alpha}, \alpha>1$ & $>20$ & Not Applicable \\
\cline { 2 - 4 } & WGN & $<15$ & $<0.35$ \\
\cline { 2 - 4 } & $1 / f$ & $15-35$ & $0.1-0.15$ \\
\hline
\end{tabular}

associated with modeling colored noise processes in BA. First, the slope of the asymptotes of ADEV of the measured height $\tilde{h}$, hence the value of $\alpha$, is significantly influenced by changes in the local air pressure (e.g. due to the approaching weather systems) as evident from the overlapping sections of ADEV graphs associated with $1 / f^{\alpha}(\alpha>1)$ type random process in Figure 3.2 and from Figure 3.4. Unfortunately, a universal mathematical model applicable to the $1 / f^{\alpha}$ colored noise processes that works for a range of values of $\alpha$ does not exist $[59,60]$. Second, while $1 / f^{2}$ colored noise processes can be simply modeled by a first order RW, large number of states are required to accurately model 
colored noise processes for $\alpha<2$ with $\alpha=1$ requiring an infinite number of states [59]. In this dissertation, colored noise processes in BA are approximated by a modified RW model, providing a tractable framework for floor localization based on smartphone sensors.

As stated earlier, the height error $\Delta_{h}$, can be expressed as the sum of two independent noise processes: a WGN process, and a colored noise process. That is

$$
\Delta_{h}[n]=b[n]+w[n]
$$

where $w \sim \mathcal{N}\left(0, \sigma_{w}\right)$, and $\sigma_{w}$ is the standard deviation of the WGN process and ranges from 0.15-0.35 $\mathrm{m}$ for the SUTs considered in this thesis (see TABLE 3.1 for more details). Also, $b[n]$ represents the randomly varying mean associated with the colored noise processes of $1 / f^{\alpha}$ PSD. From ADEV analysis, it is evident that $\Delta_{h}[n]$ can be approximated by a locally stationary random process such that $b[n]=b_{k}, n=(k-1) N_{b}+1, \ldots, k N_{b}$, $\forall k \in \mathbb{Z}^{+}$, where $N_{b}=F_{s} T_{b}$ and $\mathbb{Z}^{+}$denotes the set of positive integers. That is, the value of the bias term $b[n]$ can be assumed approximately constant during each observation interval of length $T_{b}$. This interpretation of bias instability is analogous to the definition of cluster mean in (3.3) for $\tilde{h}=\Delta_{h}$, i.e. fixed sensor location, and $\tau_{c}=T_{b}$ such that $\bar{\psi}_{k}\left(T_{b}\right)=$ $b_{k}$, which subsequently results in a first order RW model for $b_{k}$ as

$$
b_{k+m}=b_{k}+\sqrt{m} w_{b}
$$

where $w_{b}$ is zero-mean WGN with standard deviation of $\sigma_{b}=\sigma_{A D E V}\left(T_{b}\right)$, and $m T_{b}, \forall m \in$ $\mathbb{Z}^{+}$, is the time elapsed since $b_{k}$ was last determined. For the MEMS BP sensors used in this thesis, $\sigma_{b}$ lies in the range $0.075<\sigma_{b}<0.15 \mathrm{~m}$, as shown in TABLE 3.1.

By substituting (3.5) and (3.4) in (3.2), the BA signal model can be given as

$$
\tilde{h}_{k+m}[n]=h[n]+b_{k+m}+w[n]
$$

where $b_{k+m} \sim \mathcal{N}\left(b_{k}, \sqrt{m} \sigma_{b}\right)$ from (3.5). Accordingly, it follows from (3.6) that $\tilde{h}_{k+m}[n]-$ $h[n]-b_{k} \sim \mathcal{N}\left(0, \sqrt{m} \sigma_{b}+\sigma_{w}\right)$.

For further verification of measurement model, use of a first order Autoregressive Moving Average (ARMA) was made, which is shown in APPENDIX A. 


\subsection{Framework for BP-based Floor Localization}

According to (3.6), for a known bias term $b_{k}$, maximum likelihood (ML) estimation of actual height is

$$
\hat{h}[n] \mid b_{k}=\tilde{h}_{k+m}[n]-b_{k}
$$

where $\hat{h}[n] \mid b_{k}$ is the estimated height for a known $b_{k}$ with $\operatorname{var}\left(\hat{h}[n] \mid b_{k}\right)=m \sigma_{b}^{2}+\sigma_{w}^{2}$.

For notational brevity, hereafter, $\hat{h} \mid b_{k}$ will be denoted as $\hat{h}^{b}$ and consequently $\hat{h}[n] \mid b_{k}$ will be replaced with $\hat{h}^{b}[n]$.

Without access to any external information to update $b_{k}, \hat{h}^{b}$ in BA can only be used over short-term ( $<30$ minutes) measurements for floor localization. From the ADEV graphs in Figure 3.2, the deviation in $\tilde{h}$ introduced by BA is found to be less than 2 meters when the measurement length considered is less than 30 minutes. Since typically the floor heights in most of the buildings are larger than 2.5 meters, BA can be utilized for floor localization over short-term measurements without observing large errors.

On the contrary, as mentioned before, DBA can be used for floor localization over long-term measurements ( $>30$ minutes) because differencing operation in DBA mitigates the random bias introduced by approaching weather systems. Figure 3.5 shows height error $\Delta_{h}$ observed in DBA, where two BP sensors were positioned at a fixed height and in proximity during a 115 hours long measurement inside a typical office building. From the figure, $\Delta_{h}$ is observed to fluctuate to a small degree approximately centering at zero and no large deviation is detected. As such, measurement from DBA can be considered locally stationary and used for floor localization over an extended period.

When BP sensor is positioned at a fixed height, measured BP follows a Gaussian distribution [10]. As such, from the conducted measurements, it was observed that $\hat{h}^{b}$ on a fixed floor also has a Gaussian distribution. Figure 3.6 shows $\hat{h}^{b}$ measured on the first floor of TFDL, where $\hat{h}^{b}$ follows Gaussian distribution with a mean of zero. Considering the altimetry system is locally-stationary, $\hat{h}^{b}$ can be shown to have a Gaussian distribution with a different mean for all the floors inside a building. As such, floor localization using $\hat{h}^{b}$ can be considered as a multiple hypothesis testing (MHT) problem, where the distribution of 


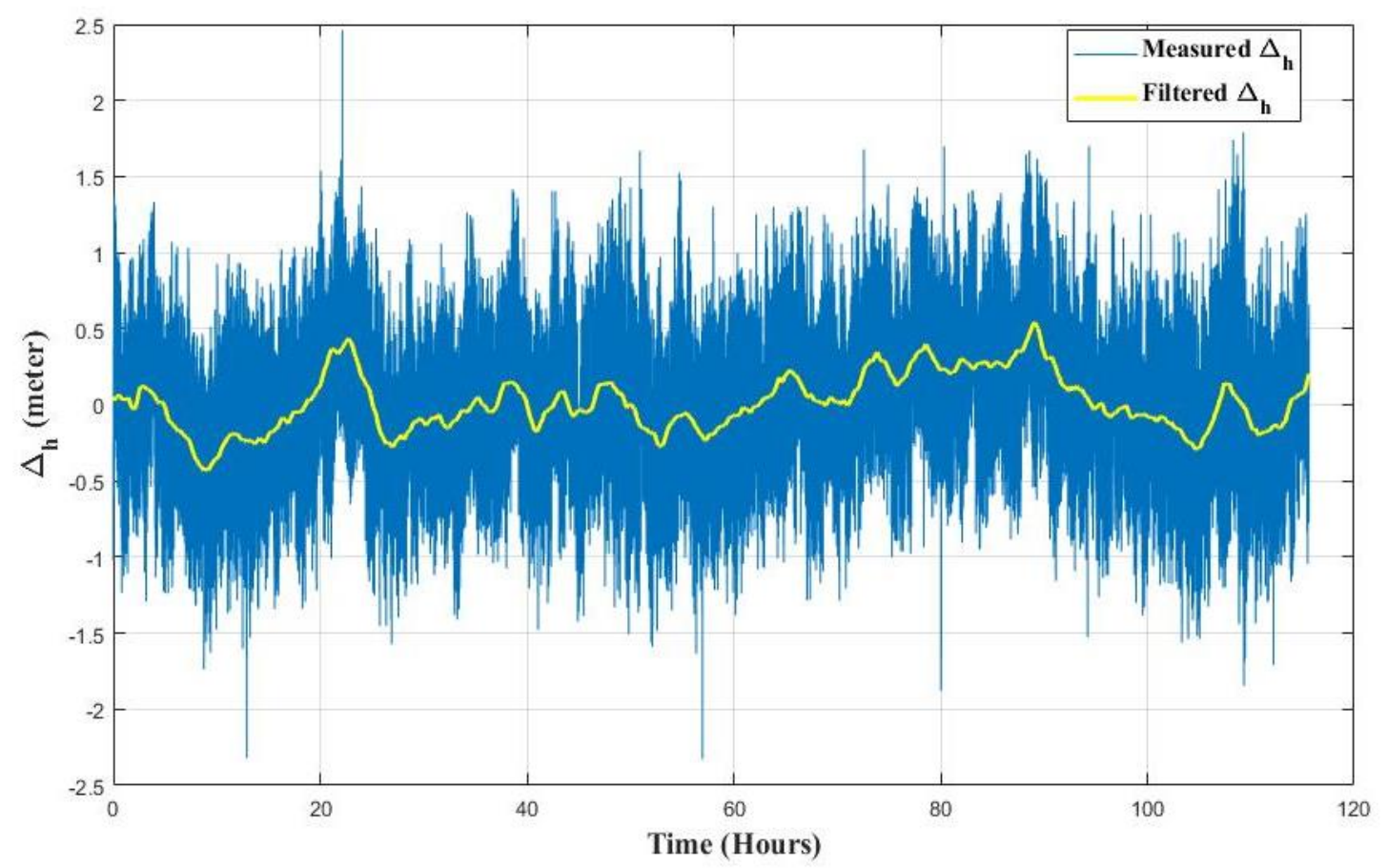

Figure 3.5: Measured height error $\Delta_{h}$ using DBA

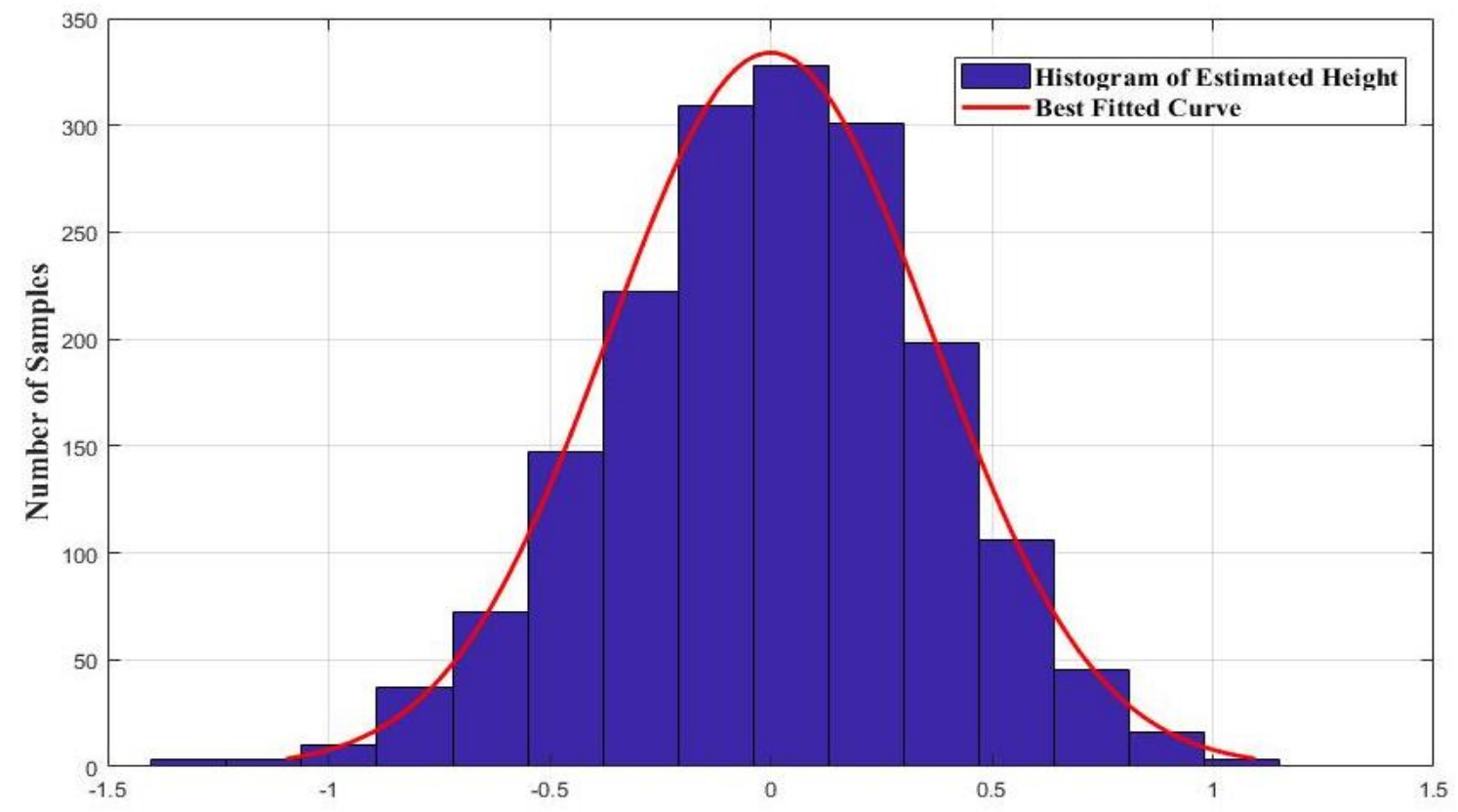

Figure 3.6: Distribution of estimated height $\hat{h}^{b}$ on a fixed floor (First floor of TFDL) 
$\hat{h}^{b}$ on each floor assist in localization. As will be shown shortly, in the context of BP-based floor localization MHT realizes a MAP classifier based on minimum probability of error criteria.

\subsubsection{Multiple Hypothesis Testing}

In MHT, each of the floor is assigned with a hypothesis and the aim is to select a solution that minimizes the Bayes risk or cost function. A cost function considers a cost or risk associated with each false detection or with each error made; and the cost value depends on the application. Let $\mathcal{L}=\left\{\ell_{1}, \ell_{2}, \ldots, \ell_{N}\right\}$ denote the set of all possible smartphone floor locations in an $N$-storey building, such as

$$
\begin{aligned}
& \ell_{1}: \widehat{h}^{b}[n]=\bar{h}_{1}+w[n] \quad n=0,1,2, \ldots, M-1 \\
& \ell_{2}: \widehat{h}^{b}[n]=\bar{h}_{2}+w[n] \quad n=0,1,2, \ldots, M-1 \\
& \ell_{3}: \widehat{h}^{b}[n]=\bar{h}_{3}+w[n] \quad n=0,1,2, \ldots, M-1 \\
& \ell_{N}: \widehat{h}^{b}[n]=\bar{h}_{N}+w[n] \quad n=0,1,2, \ldots, M-1
\end{aligned}
$$

where $\ell_{f} \in \mathcal{L}$ denotes the hypothesis associated with $f$-th floor, $\bar{h}_{f}$ is the correct floor height corresponding to $\ell_{f}$ and is known a priori, $M$ is the total number of measurement samples, and $w$ was defined earlier in Section 3.2. $\bar{h}_{f}$ can be determined from a building's architectural plan or by using a Laser range meter, and in this thesis, $\bar{h}_{f}$ (i.e. correct floor height corresponding to $\ell_{f}$ ) is considered as the average of the minimum and maximum height associated with floor $\ell_{f}$. If $P\left(\ell_{f}\right)$ denotes the prior probability of occurring hypothesis $\ell_{f}$ and $P\left(\ell_{f} \mid \ell_{l}\right)$ represents the conditional probability of deciding hypothesis $\ell_{f}$ when $\ell_{l}$ is true, then Bayes risk is defined as [61]

$$
\mathcal{R}=\sum_{f=1}^{N} \sum_{l=1}^{N} C_{f l} P\left(\ell_{f} \mid \ell_{l}\right) P\left(\ell_{l}\right)
$$


where $\mathcal{R}$ is the Bayes risk, and $C_{f l}$ is the assigned cost to the scenario where $\ell_{f}$ is selected as the correct hypothesis, but $\ell_{l}$ is true.

Moreover, according to [61], for correct floor selection, a hypothesis should be chosen that minimizes

$$
C_{f}\left(\hat{h}^{b}[n]\right)=\sum_{l=1}^{N} C_{f l} P\left(\ell_{l} \mid \hat{h}^{b}[n]\right) ; f=1,2, \ldots, N
$$

where $C_{f}\left(\hat{h}^{b}[n]\right)$ is the average cost of deciding $\ell_{f}$ and $P\left(\ell_{l} \mid \hat{h}^{b}[n]\right)$ is the posteriori probability of selecting hypothesis $\ell_{l}$, provided data $\widehat{h}^{b}[n]$ is observed.

As all the hypotheses associated with the floors inside a building have the same probability of occurring and as no specific cost has to be assigned to any particular floor, it is safe to assume that for $f=l, C_{f l}=0$ or otherwise, $C_{f l}=1$. As a result, $\mathcal{R}$ simplifies to $P_{e}$, where $P_{e}$ denotes the probability of error in detection. Then according to (3.9), $C_{f}\left(\hat{h}^{b}[n]\right)$ becomes

$$
\begin{aligned}
C_{f}\left(\hat{h}^{b}[n]\right)=\sum_{\substack{l=1,2, \ldots, N \\
l \neq f}} P\left(\ell_{l} \mid \hat{h}^{b}[n]\right) \\
\quad=\sum_{l=1}^{N} P\left(\ell_{l} \mid \hat{h}^{b}[n]\right)-P\left(\ell_{f} \mid \hat{h}^{b}[n]\right) ; f=1,2, \ldots, N
\end{aligned}
$$

As only the second term depends on $f, C_{f}\left(\hat{h}^{b}[n]\right)$ is minimized by maximizing $P\left(\ell_{f} \mid \hat{h}^{b}[n]\right)$. Thus, to obtain minimum $P_{e}$, decision rule decides $\ell_{f}$ if

$$
P\left(\ell_{f} \mid \hat{h}^{b}[n]\right)>P\left(\ell_{l} \mid \hat{h}^{b}[n]\right) ; l \neq f
$$

(3.11) aims to maximize the posteriori probability for making decision and this is known as $M$-ary MAP decision rule. Now, as the prior probability of being on a floor is considered equal for all of the floors, $P\left(\ell_{f} \mid \hat{h}^{b}[n]\right)$ is expressed as [61] 


$$
\begin{aligned}
P\left(\ell_{f} \mid \hat{h}^{b}[n]\right) & =\frac{P\left(\hat{h}^{b}[n] \mid \ell_{f}\right) p\left(\ell_{f}\right)}{P\left(\hat{h}^{b}[n]\right)} \\
& =\frac{P\left(\hat{h}^{b}[n] \mid \ell_{f}\right) \cdot \frac{1}{N}}{P\left(\hat{h}^{b}[n]\right)}
\end{aligned}
$$

where $\frac{1}{N}$ is the prior probability of being on a floor when a building with $N$ number of floors is considered (i.e. it is equally likely to be on any of the $N$ floors). Also, since $P\left(\hat{h}^{b}[n]\right)$ in (3.12) is not a function of $f$, it would suffice to maximize $P\left(\hat{h}^{b}[n] \mid \ell_{f}\right) P\left(\ell_{f}\right)$ to equivalently maximize $P\left(\ell_{f} \mid \hat{h}^{b}[n]\right)$. Hence, for hypotheses with equal prior probabilities, decision rule decides $\ell_{f}$ if

$$
\begin{gathered}
P\left(\hat{h}^{b}[n] \mid \ell_{f}\right)>P\left(\hat{h}^{b}[n] \mid \ell_{l}\right) ; l \neq f \\
\text { or, } p\left(\hat{h}^{b}[n] \mid \ell_{f}\right)>p\left(\hat{h}^{b}[n] \mid \ell_{l}\right) ; l \neq f
\end{gathered}
$$

where $p($.$) is the probability density function (PDF) or likelihood function, p\left(\hat{h}^{b}[n] \mid \ell_{f}\right)$ is the conditional PDF. (3.13) is known as M-ary ML decision rule.

The conditional PDF $p\left(\hat{h}^{b}[n] \mid \ell_{f}\right)$ is expressed as [61]

$$
p\left(\hat{h}^{b}[n] \mid \ell_{f}\right)=\frac{1}{\sqrt{2 \pi \sigma^{2}}} \exp \left[-\frac{1}{2 \sigma^{2}}\left(\hat{h}^{b}[n]-\bar{h}_{f}\right)^{2}\right] ; f=1,2, \ldots, N
$$

From (3.14), it can be realized that to maximize $p\left(\hat{h}^{b}[n] \mid \ell_{f}\right)$, it is required to minimize $\left(\hat{h}^{b}[n]-\bar{h}_{f}\right)^{2}$. Hence, a MAP classifier based on minimum probability of error criteria is used to estimate mobile user floor location as [61]

$$
\ell_{\mathrm{MAP}}=\underset{\ell_{f} \in \mathcal{L}}{\arg \min }\left[\left(\widehat{h}^{b}[n]-\bar{h}_{f}\right)^{2}\right]
$$

\subsection{Experimental Setup}

The measurements performed for the research presented in this dissertation were designed to evaluate floor localization based on BP and Wi-Fi RSS readings in different 


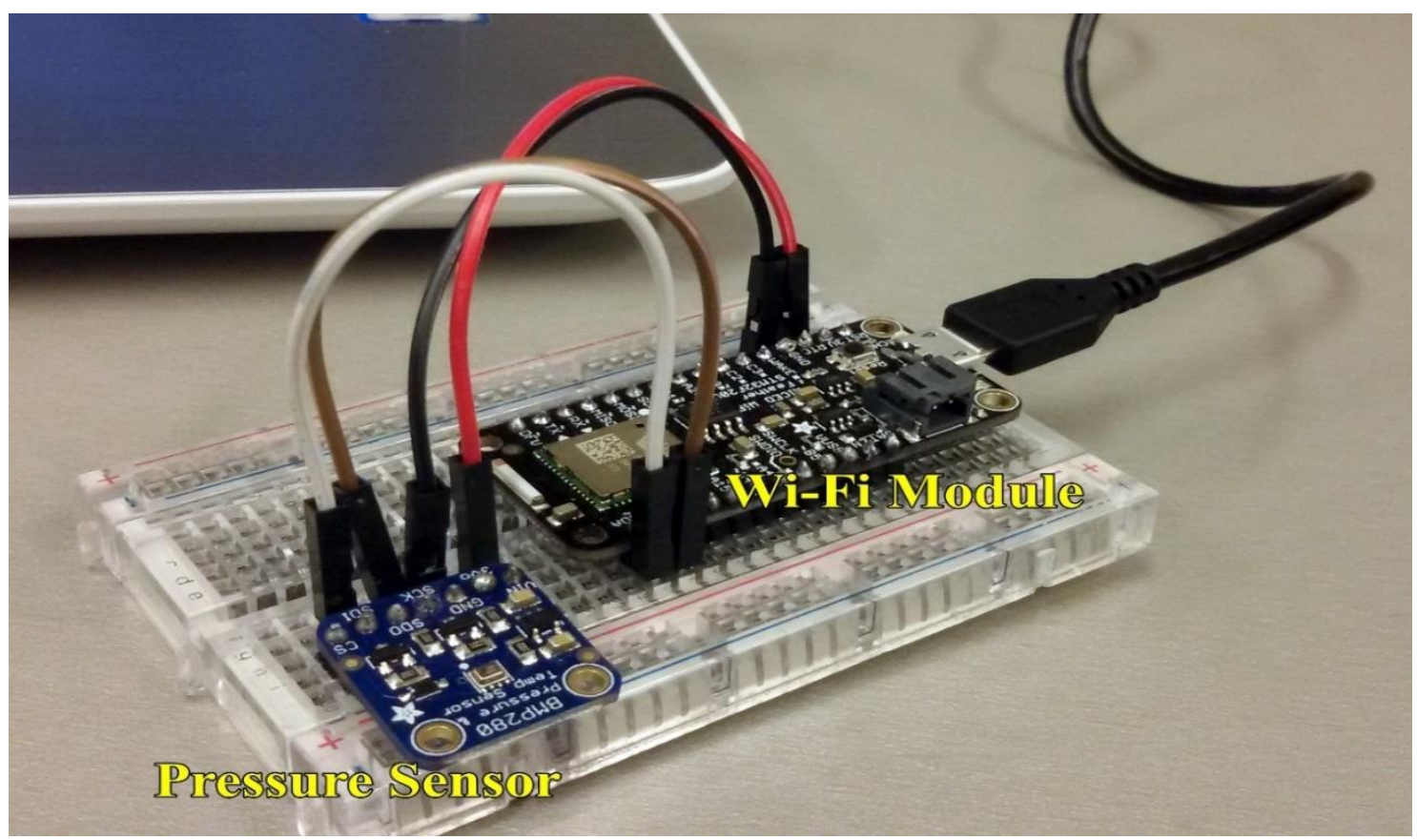

Figure 3.7: Handheld electronic comprising BMP280 and Wi-Fi module

indoor environments. This section provides an overview of the measurement apparatus and the selected environments for measurements.

\subsubsection{Apparatus}

For Wi-Fi RSS measurements, Arduino Adafruit WICED Feather Wi-Fi was used, which has an embedded chip BCM43362 (later, renamed as CYW43362). BCM43362 is a chip with an integrated $2.4 \mathrm{GHz}$ RF transceiver that supports the IEEE $802.11^{\mathrm{TM}} \mathrm{b} / \mathrm{g} / \mathrm{n}$ standards [62]. BCM43362 can operate within the range of -30 to $+85^{\circ} \mathrm{C}$ (ambient temperature) and has a resolution of $1 \mathrm{dBm}$. To measure barometric pressure, BP sensor Adafruit BMP280 was integrated into the system. The range of pressure measurement for BMP280 is 300 to $1100 \mathrm{hPa}$, which is equivalent to $-500 \mathrm{~m}$ below the sea level to $9000 \mathrm{~m}$ above the sea level [55]. In addition, BMP280 can operate within the temperature range of -40 to $+85^{\circ} \mathrm{C}$ and has a resolution of $0.0016 \mathrm{hPa}$ (equivalent to $0.015 \mathrm{~m}$ ). A sample rate of $1 \mathrm{~Hz}$ was maintained during all measurement runs. The complete handheld electronic device comprising BP sensor BMP280 and Wi-Fi module (i.e. Arduino Adafruit WICED Feather Wi-Fi) that was carried during the measurement runs is shown in Figure 3.7. In the 
post-analysis stage, MATLAB (Version R2017a) was used to analyze collected data and to generate graphs.

\subsubsection{Selected Environments for Measurements}

Three multi-storey buildings were considered on the University of Calgary campus grounds for measurements. These buildings were selected due to their different structural designs, which help to evaluate the robustness of a localization method. Measurements were carried out on different days to test floor localization methods under different weather patterns.

Building 1:

The first measurement run was conducted inside Information and Communications Technology (ICT) building, which is typical of a multi-story building with a combination of open and office floor plans. The first three floors were considered for the measurements. The ground floor covers an area of approximately 2250 sq. meters and has an open layout with shops, sitting area, and large lecture halls. The second and third floors cover an area of approximately 1950 sq. meters and comprise a variety of offices and labs connected by narrow hallways. The ceiling on the ground floor is 7 meters high and the ceilings on the second and third floors are around 4 meters high. The measurement was carried out on June 19 of 2017 and it lasted for 1 hour and 45 minutes. The exterior and interior views of the ICT building are shown in Figure 3.8.

\section{Building 2:}

The second measurement was conducted inside Taylor Family Digital Library (TFDL), which is a six-story building with open floor designs. The use of concrete slabs and signal interfering metal mesh was made to separate two adjacent floors in TFDL. The first two floors have an area of 5370 sq. meters and the rest of the floors individually cover 3,470 sq. meters. Bookshelves and study areas occupy most of the areas on each floor and there is a hollow interior between first and second floors. On average, the ceilings on all floors are roughly 5 meters high. The measurement was carried out on August 8, 2017, and it lasted for 2 hours. Figure 3.9 shows the outer and inner views of TFDL. 


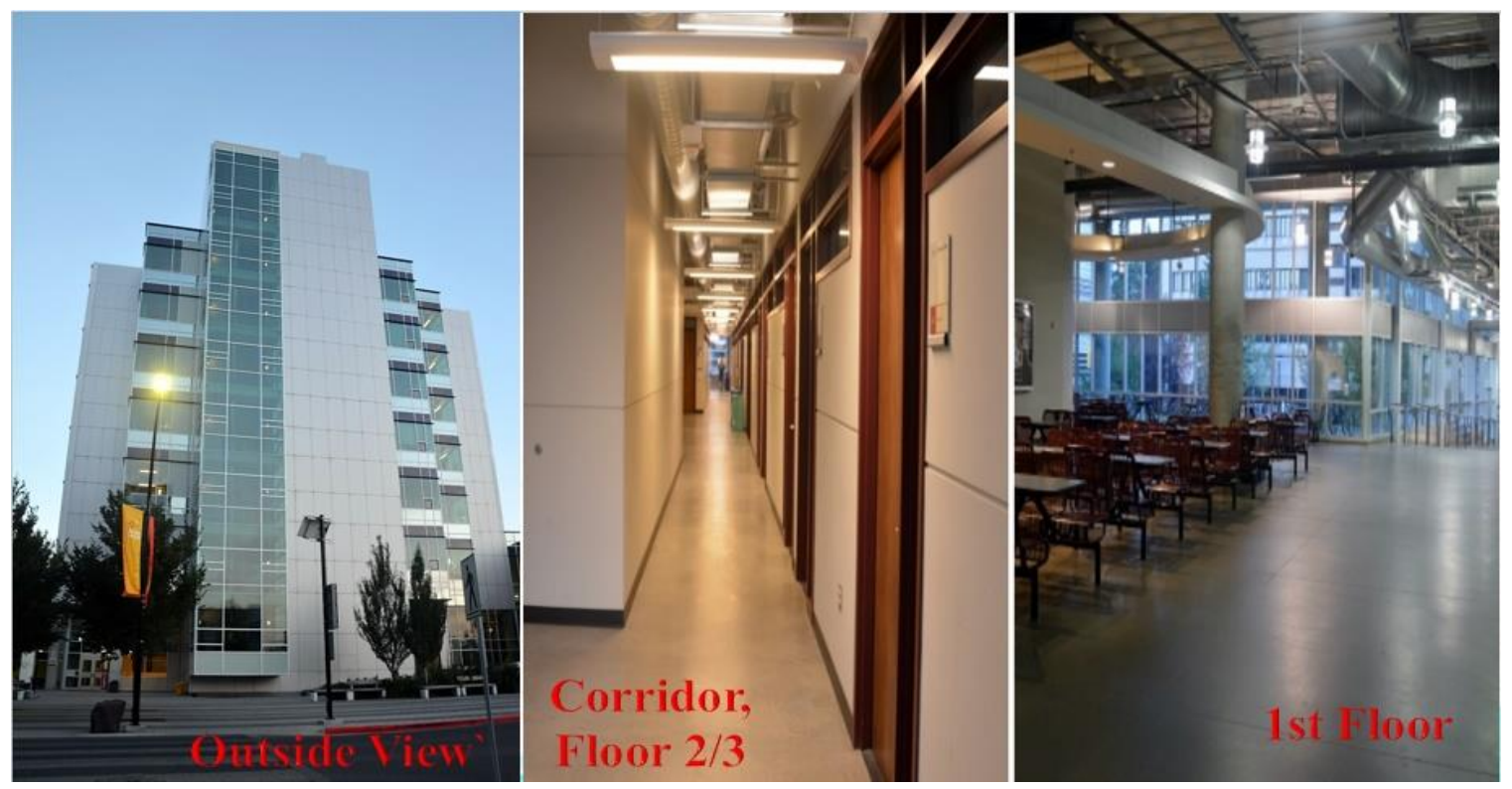

Figure 3.8: Left figure - Outside view of ICT building; Middle figure - Narrow hallway on the second/third floor; Right figure - Partial view of the first floor (open floor plan)

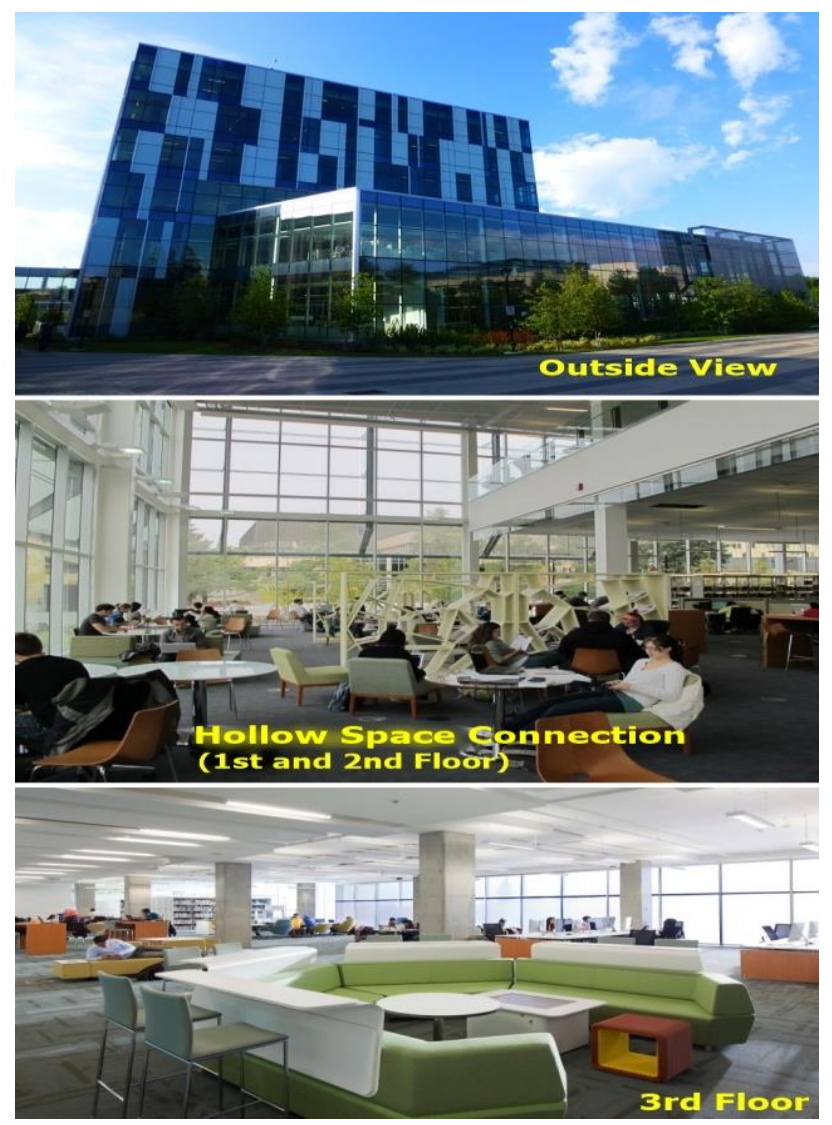

Figure 3.9: Top figure - Outside view of TFDL building; Middle figure - Hollow interiors between first and second floor; Bottom figure - Partial view of the third floor 


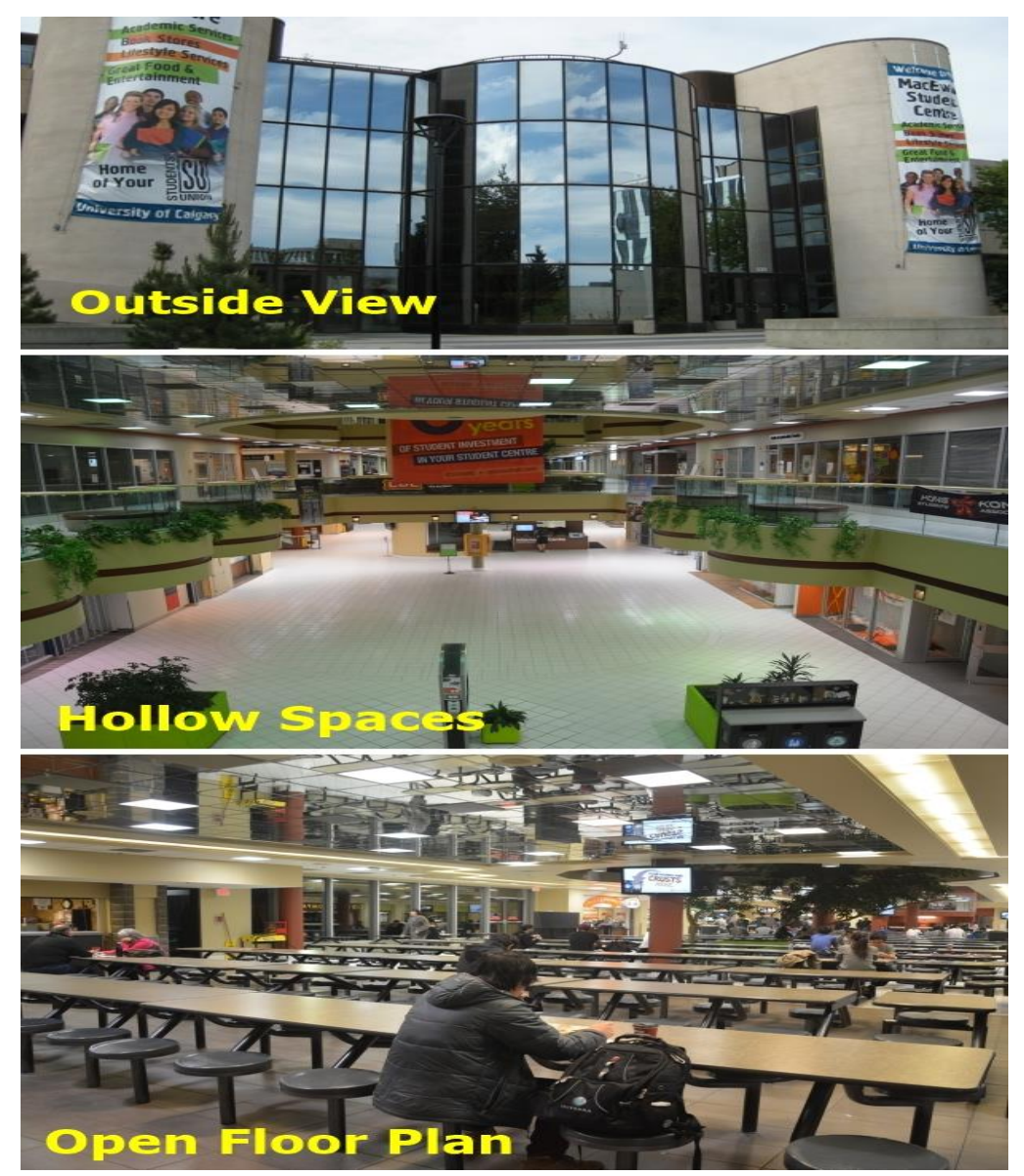

Figure 3.10: Top figure - Outside view of MSC; Middle figure - Hollow interiors inside MSC; Bottom Figure - Open floor plan on the first floor of MSC

\section{Building 3:}

The third measurement was conducted inside MacEwan Student Centre (MSC), which is typical of a modern mall with an open multi-level design with large hollow spaces and has many food shops and offices. As a result, RSS measurements are highly affected by multipaths or Line-of-sight (LOS) signals from the APs located on other floors. The first and the second floor have an area of approximately 5400 sq. meters and the third and fourth floors cover approximately 5300 and 3700 sq. meters, respectively. The ceilings on each floor are around 4 meters high. The measurement lasted roughly 2 hours and it was conducted on August 17, 2017. Figure 3.10 exhibits the outside and inside views of MSC. 


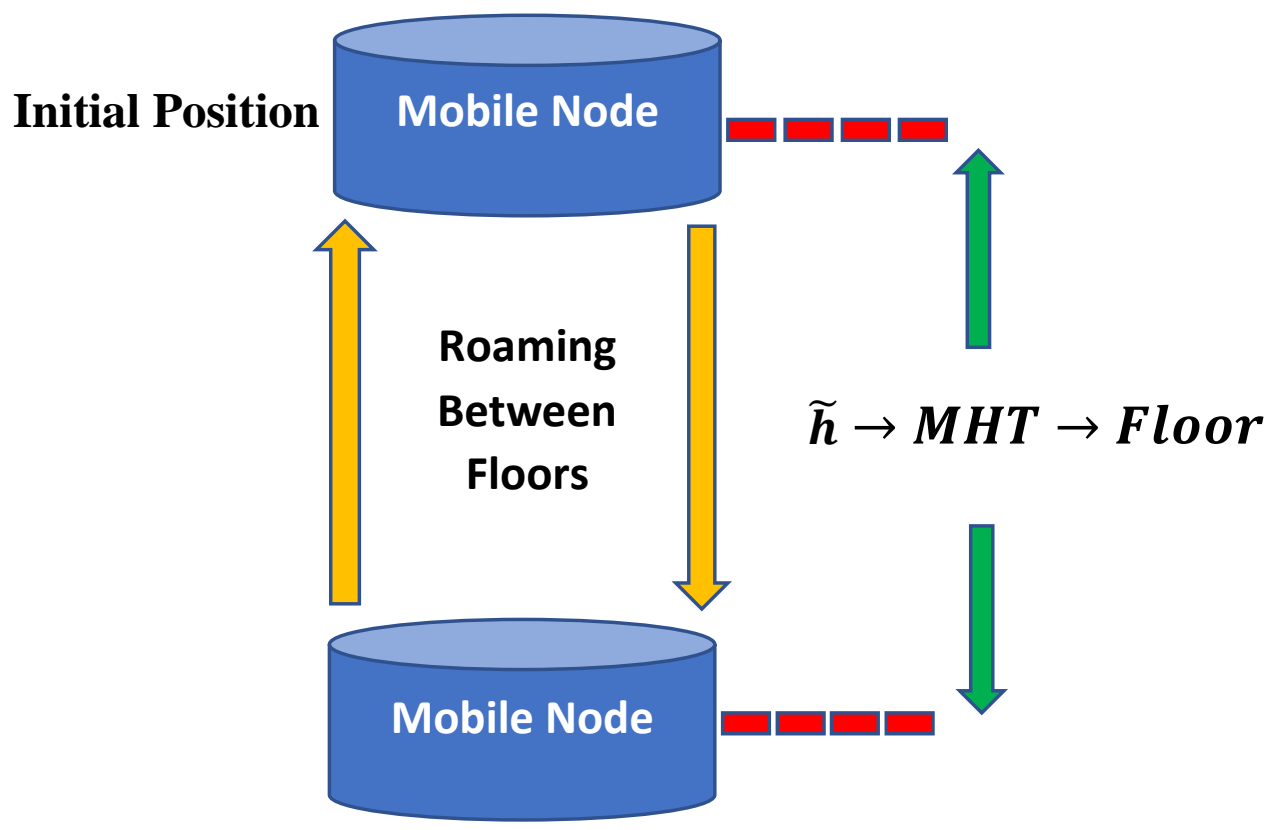

Figure 3.11: BA-based floor localization system

\subsection{Floor Localization Using BA}

In BA-based floor localization, it is assumed that initially the user remains on the same floor while a total of $M$ BP measurements are captured. The average of these initial $M$ measurements, denoted by $\mathcal{P}_{r}$, and the equivalent height, denoted by $h_{r}$, hereafter termed as reference pressure and reference height, respectively. $h_{r}$ is used for initial calibration, i.e., any floor identification thereafter is made relative to the floor identification that corresponds to $h_{r}$. The floor or place where the initial measurements are taken is referred to as the reference point. To determine the floor position of a mobile user at time $n$, the height $\left(h_{m}\right)$ corresponding to the pressure of that location is determined. Afterwards, $h_{m}$ is compared with $h_{r}$ to obtain $\tilde{h}$, i.e.

$$
\tilde{h}=h_{m}-h_{r}
$$

Using $\tilde{h}, \hat{h}^{b}$ is determined and then MAP classifier based on minimum probability of error criteria is used for estimating user floor location. Figure 3.11 shows a diagram of BA-based floor localization system. 


\subsubsection{Results and Analysis of BA-based Floor Localization}

To analyze the performance of BA-based floor localization, BP readings were measured inside all three buildings (mentioned in Section 3.4.2) while a mobile user roamed between floors carrying the handheld electronic device shown in Section 3.4.1. Figure 3.12 presents estimated height $\hat{h}^{b}$ based on BA and user correct floor location (dashed line) during the measurements. As can be seen, $\hat{h}^{b}$ began to deviate from its true value for-long term measurements (i.e. $\geq 30$ minutes) due to local variations of air pressure, $b_{k}$. It can also be observed from the figure that for a given period, deviation introduced in $\hat{h}^{b}$ was dependant on rate of change in diurnal weather. Deviation of 3,4 , and 5 meters were observed after 1 hour of measurement in ICT, TFDL, and MSC, respectively.

To further observe the impact of local weather change on measurement deviation, Figure 3.13 was generated. Figure 3.13 demonstrates the distribution of $\hat{h}^{b}$ on the first floor of TFDL, where measurements were taken 1.5 hours apart on the same day. From the figure, a shift in the mean of the distribution of $\hat{h}^{b}$ is observed, which is the result of changes in the local air pressure (i.e. weather) over time. As such, a deterioration was observed in floor localization accuracy when MAP classifier based on minimum probability of error criteria (see Section 3.3 for more details) was applied for floor identification. Floor localization accuracy is defined here as the ratio (in percentage) of correct to total floor identifications.

TABLE 3.2 shows the floor localization accuracy using BA for different lengths of measurements. For measurement length of less than 5 minutes, $100 \%$ accuracy was achieved because the deviation in $\hat{h}^{b}$ within that period did not have any noticeable impact on floor localization. Whereas, as can be seen from TABLE 3.2, BA-based floor localization is highly unreliable over long-term. For instance, BA-based floor localization accuracy of less than $60 \%$ was measured when test durations of $>1.5$ hours were considered.

From the above results, it is concluded that due to deviation observed in BA, large errors are introduced in floor localization for long-term measurements. As such, BA cannot be used as a stand-alone method for floor localization. 

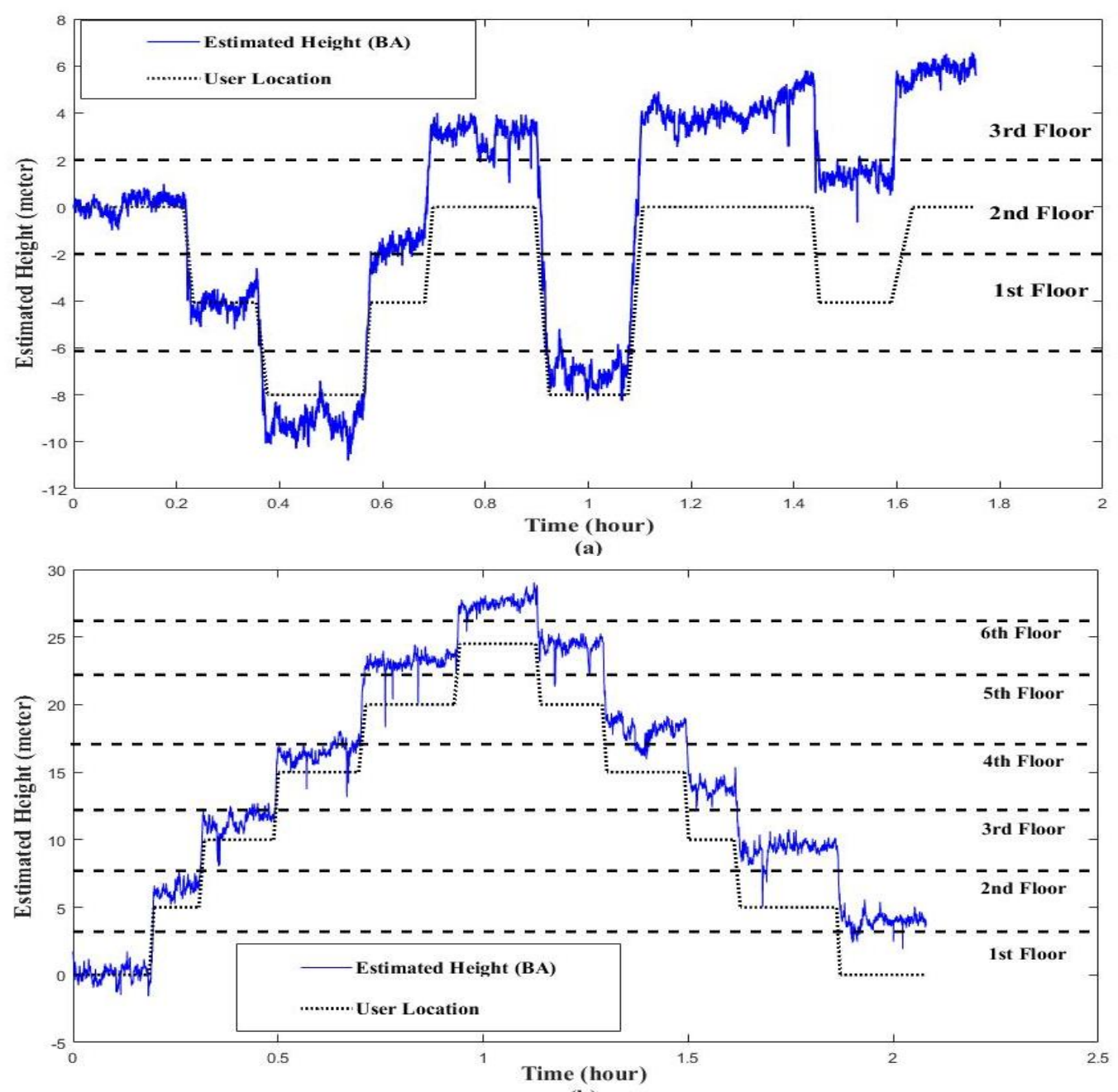

(b)

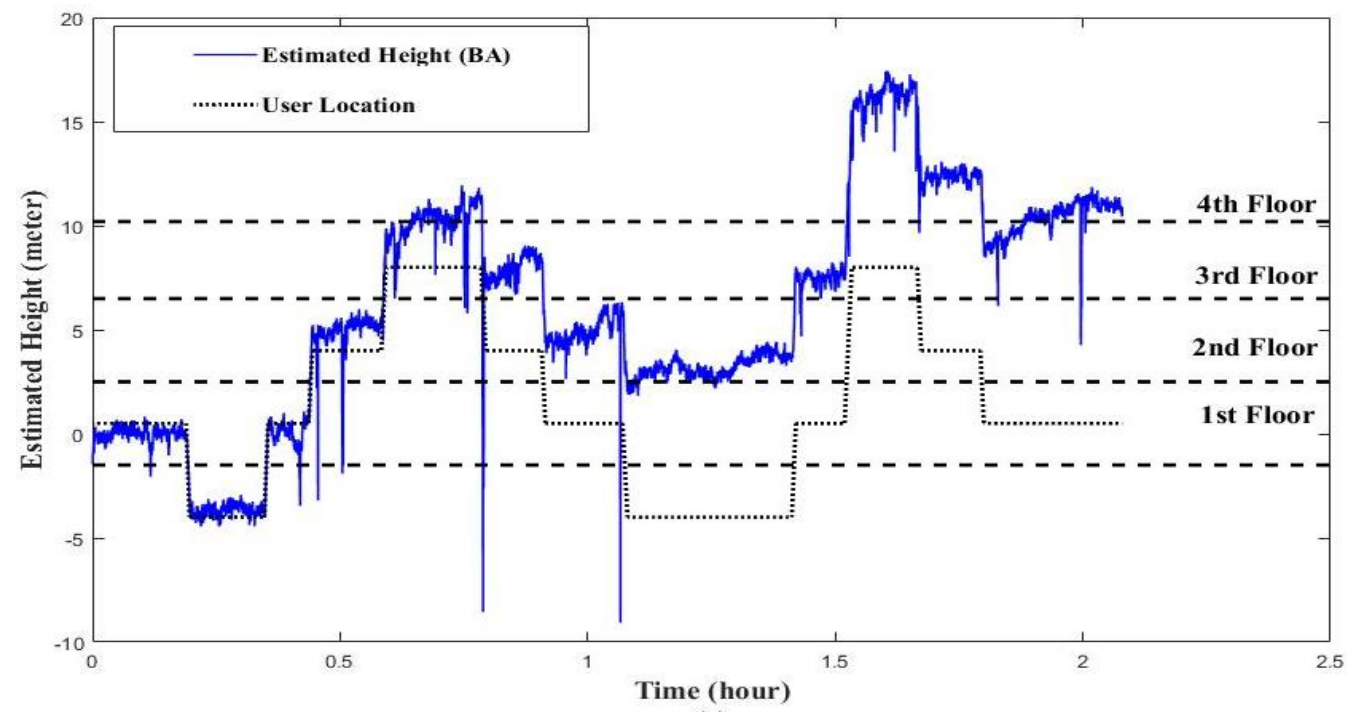

(c)

Figure 3.12: Estimated height using BA in (a) ICT, (b) TFDL, and (c) MSC 


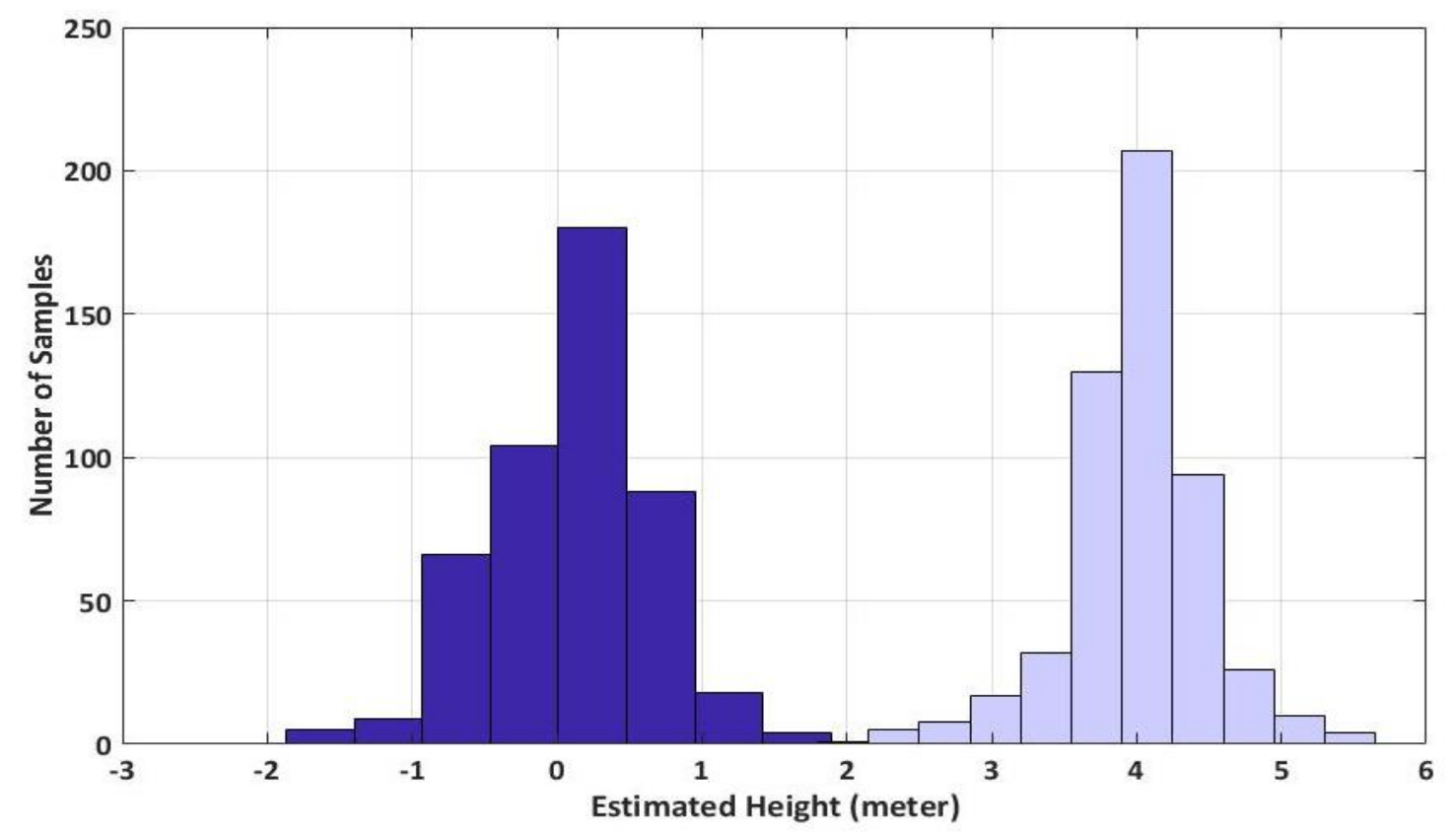

Figure 3.13: Distribution of estimated height $\hat{h}^{b}$ (1.5 hours apart) on the first floor of TFDL

TABLE 3.2

FLOOR LOCALIZATION ACCURACY USING BA

\begin{tabular}{|c|c|c|c|c|}
\hline $\begin{array}{c}\text { Measurement } \\
\text { Length }\end{array}$ & $\begin{array}{c}\text { ICT } \\
(\text { June 19) }\end{array}$ & $\begin{array}{c}\text { TFDL } \\
\text { (August } 8)\end{array}$ & $\begin{array}{c}\text { MSC } \\
\text { (August 17) }\end{array}$ & Average \\
\hline$<5$ minutes & $100 \%$ & $100 \%$ & $100 \%$ & $100 \%$ \\
\hline$<30$ minutes & $\approx 100 \%$ & $>98 \%$ & $>99 \%$ & $>99 \%$ \\
\hline$>1.5$ hours & $<56 \%$ & $<62 \%$ & $<59 \%$ & $<59 \%$ \\
\hline
\end{tabular}

\subsection{Floor Detection Using DBA}

To overcome the challenges in BA-based floor localization, researchers introduced use of differential barometric altimetry for floor localization. The procedure for floor detection using DBA is similar to the procedure that is followed in BA except that two pressure sensors are used here. An actual pressure sensor is used to provide real time values of $\mathcal{P}_{\mathrm{r}}$ and, consequently, $h_{r}$. The sensor that measures $\mathcal{P}_{\mathrm{r}}$ is known as reference barometer/node and it is kept fixed at a reference location. Another pressure sensor (referred as mobile barometer/node) is carried by a pedestrian. 


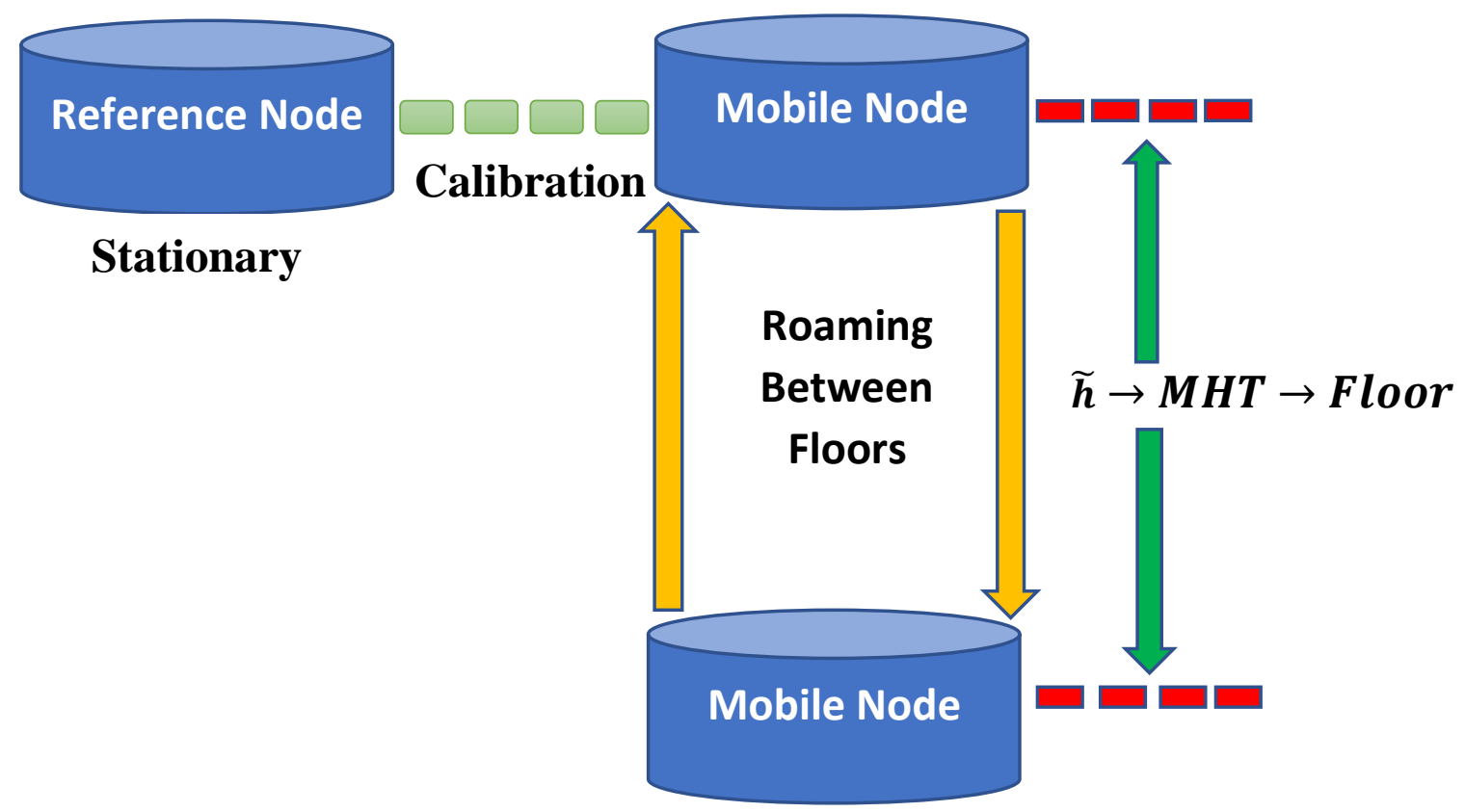

Figure 3.14: DBA-based floor localization system

The rationale behind using DBA is that if the changes in local weather influence the measured BP, then it will influence the output of both mobile and reference barometers in a similar way. As a result, real-time differencing of measured air pressures from mobile and reference barometers negates the effect of changes in weather. Nevertheless, DBA requires additional infrastructure, e.g. installation of reference nodes and ad-hoc network for real-time information exchange, hence not readily applicable.

Implementation of DBA requires a calibration between the reference and the mobile sensors to remove any existing offset. The calibration is performed by initially keeping both sensors at the same height or using crowdsourcing approach. Depending on the design, the system can have one or multiple reference barometer(s) installed on fixed floor(s). Similar to BA-based floor localization, (3.16) determines $\tilde{h}$ in DBA, and subsequently MAP classifier based on minimum probability of error criteria is used to estimate user floor location. Figure 3.14 illustrates floor localization system using DBA. 


\subsubsection{Results and Analysis of DBA-based Floor Localization}

For DBA, the same set of measurements collected by the mobile user (shown in Figure 3.12) is considered along with the $\mathrm{BP}$ readings from a reference barometer in all three buildings. During measurement, the reference barometer was kept fixed at a stationary position on the initial floor. In the post-analysis, MAP classifier in (3.15) was applied to DBA measurements for floor localization.

Figure 3.15 demonstrates the estimated height $\hat{h}^{b}$ using DBA in all three buildings, and as can be seen, unlike BA, DBA provides reliable results. Any variation in local pressure (i.e. $b_{k}$ ) had similar influence on both reference and mobile barometer output. Hence, by considering the difference of the measured BP by both barometers, $b_{k}$ was removed from the measurements. Figure 3.16 shows that as DBA mitigated the effect of $b_{k}$ (i.e. drift in air pressure) from the measurements, average $\hat{h}^{b}$ (measurement from TFDL is considered in this case) is different for different floors. Also, the distribution corresponding to each floor was well-separated from the distributions of $\hat{h}^{b}$ from other floors. As such, use of DBA in MAP classifier resulted in high floor localization accuracy.

TABLE 3.3 shows the DBA-based floor localization accuracy for different measurement lengths. As can be seen, for both short-term and long-term measurements, DBA provides highly accurate floor localization. Even for a measurement period of more than 1.5 hours, DBA achieved an accuracy of more than $97 \%$ on average. Comparing TABLE 3.3 with TABLE 3.2, it can be concluded that with the cost of additional infrastructure (e.g. installation of reference nodes and ad-hoc network for real-time information exchange), performance of DBA surpasses that of BA in floor localization. Moreover, floor localization using DBA can be used for long-term measurements.

\subsection{Summary}

In this chapter, a barometer measurement model was formulated based on extensive measurements and Allan deviation technique. Afterwards, a framework for floor localization using barometric pressure was shown. Further, two floor localization methods 


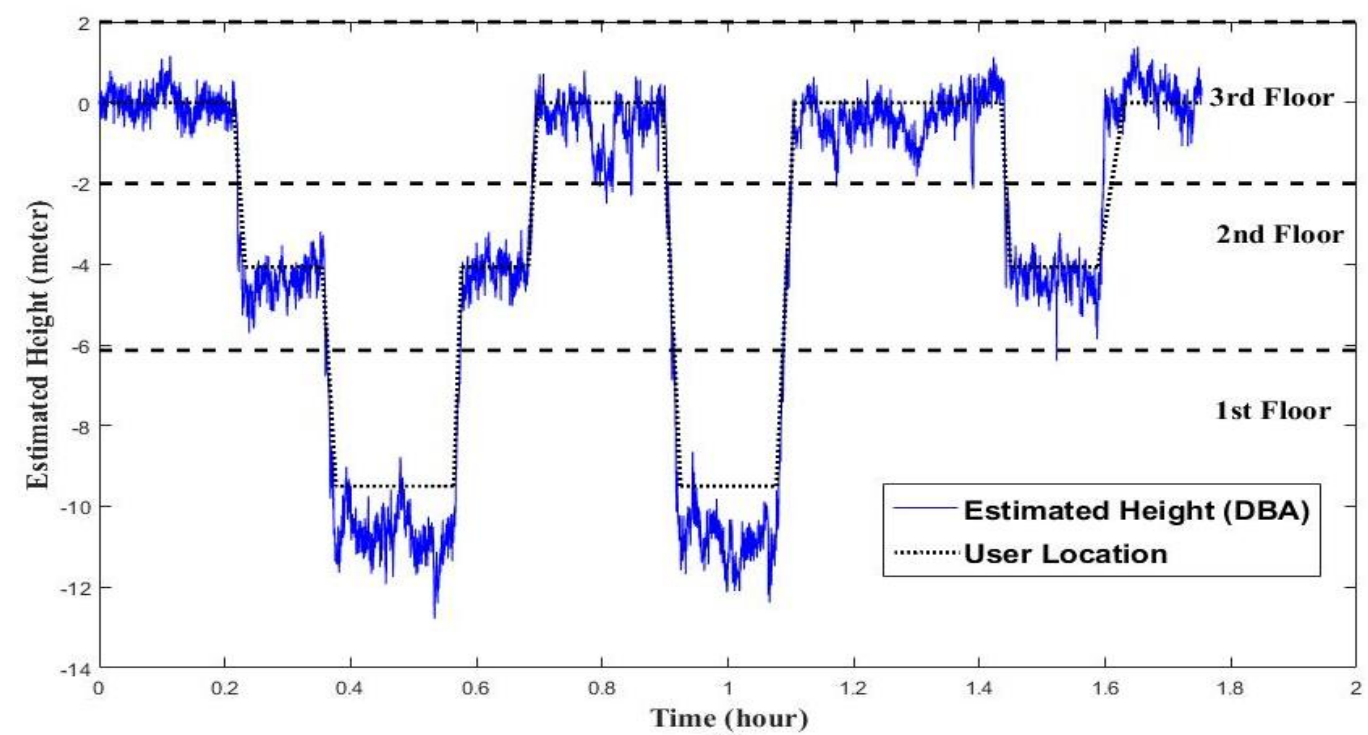

(a)

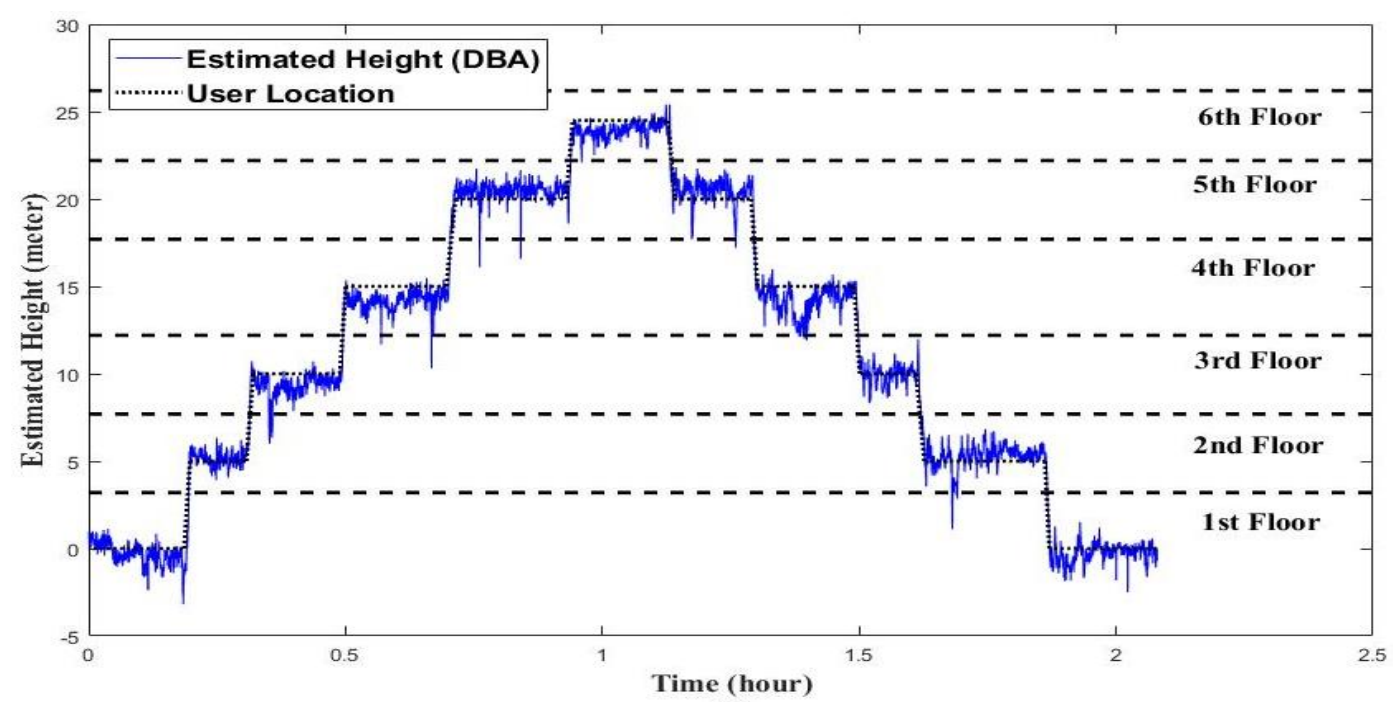

(b)

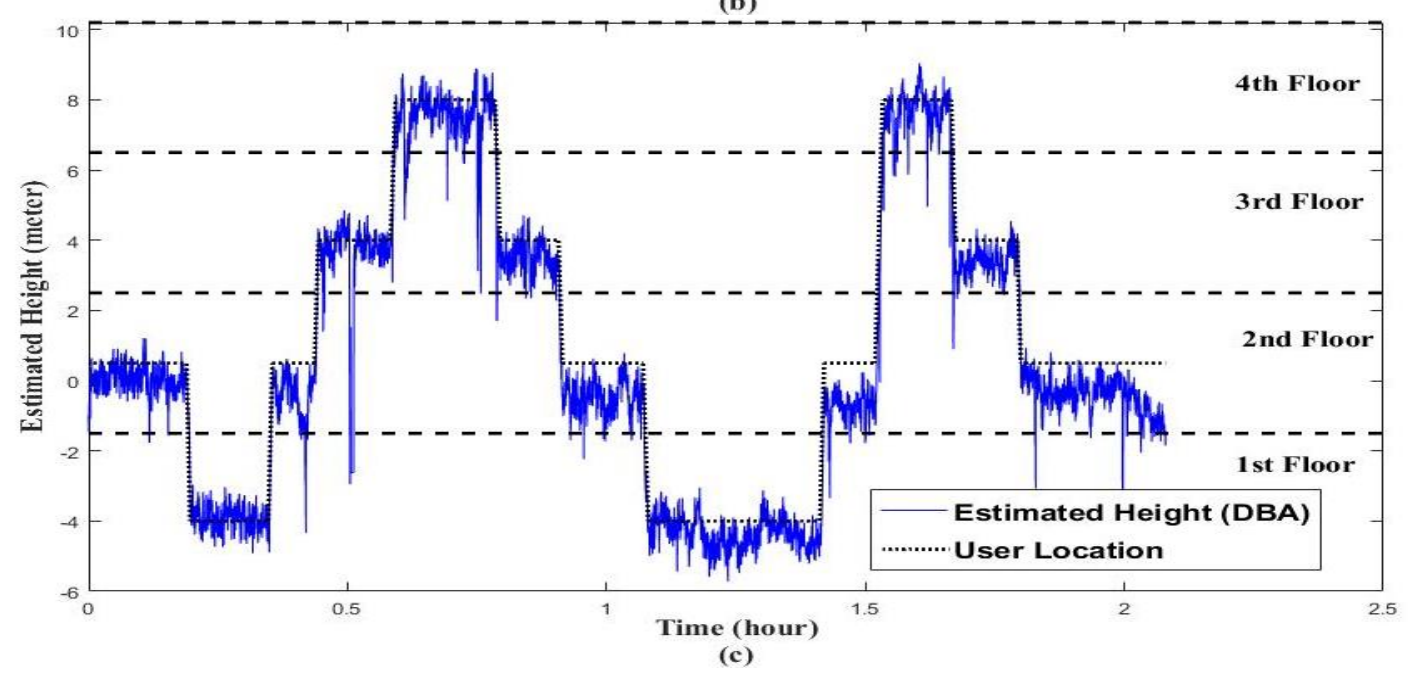

Figure 3.15: Estimated height using DBA in (a) ICT, (b) TFDL, and (c) MSC 


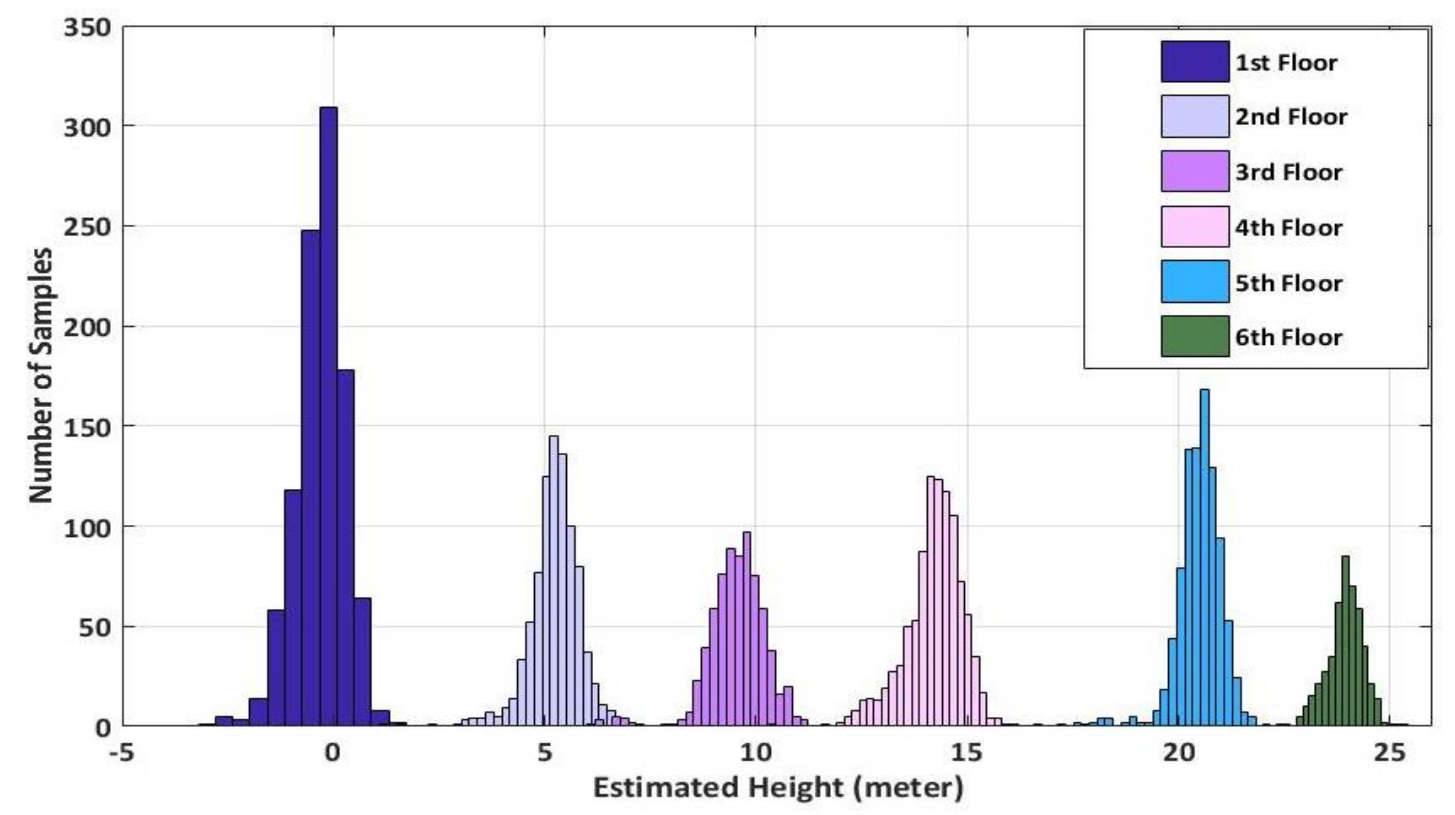

Figure 3.16: Distribution of estimated height $\hat{h}^{b}$ using DBA on different floors of TFDL

TABLE 3.3

FLOOR LOCALIZATION ACCURACY USING DBA

\begin{tabular}{|c|c|c|c|c|}
\hline $\begin{array}{l}\text { Measurement } \\
\text { Length }\end{array}$ & ICT (June 19) & $\begin{array}{c}\text { TFDL } \\
\text { (August 8) }\end{array}$ & $\begin{array}{c}\text { MSC } \\
\text { (August 17) }\end{array}$ & Average \\
\hline$<5$ minutes & $100 \%$ & $100 \%$ & $100 \%$ & $0 \%$ \\
\hline$<30$ minutes & $100 \%$ & $>99 \%$ & $>99 \%$ & $>99 \%$ \\
\hline$>1.5$ hours & $>99 \%$ & $>99 \%$ & $>99 \%$ & $>99 \%$ \\
\hline
\end{tabular}

based on measuring pressure, i.e. barometric altimetry and differential barometric altimetry, were discussed and the performance analysis of implementing those two methods was performed. It is concluded that the BA-based floor localization system can be readily implemented with state-of-the-art smartphones. Nevertheless, BA is prone to drift errors due to variations in approaching weather, which introduces large errors in floor localization for long-term measurements. As an alternative, DBA is an attractive solution to compensate for the errors introduced by the local weather. However, DBA requires installation of additional infrastructure, which makes it infeasible to implement the DBAbased localization system on a global scale. 


\section{Chapter 4: Floor Localization Using Received Signal Strength}

\subsection{Introduction}

In many commercial and industrial location applications, the major objective of the system designer is to reduce system requirements despite reasonable degradation in the positioning accuracy. For that purpose, received signal strength (RSS)-based floor localization is an attractive approach due to its cost-effectiveness, low-complexity, and pervasiveness of mobile devices all over the world [63]. Most of the RSS-based approaches can be readily implemented and incorporated into a hybrid localization approach with the existing infrastructure, e.g., access points, smartphones. Nevertheless, the localization process based on RSS is affected by multipath signals and improvement is required inside buildings with irregular structures and hollow interiors.

This chapter develops and evaluates a Monte Carlo Bayesian Inference (MCBI) framework for floor localization. The remainder of the chapter is organized as follows. Section 4.1 presents RSS distribution and its statistical characteristics based on the performed measurements inside three multi-storey buildings. Conventional RSS-based localization approaches are briefly described in Section 4.3. In Section 4.4, a MCBI algorithm is formulated for floor localization. Section 4.5 analyzes measured Wi-Fi RSS readings and evaluates the performance of the formulated algorithm in floor localization. Summary is given in Section 4.6

\subsection{RSS Distribution Model}

To design a robust estimator that can use Wi-Fi RSS from APs for floor localization, it is essential to know about the RSS distributions through wireless channels in an indoor environment. The wireless channel refers to the physical space or media through which signal propagates. The natural signal power decay and the physical obstacles (e.g., walls, doors, furniture, etc.) occupying the environment are taken into consideration to determine 


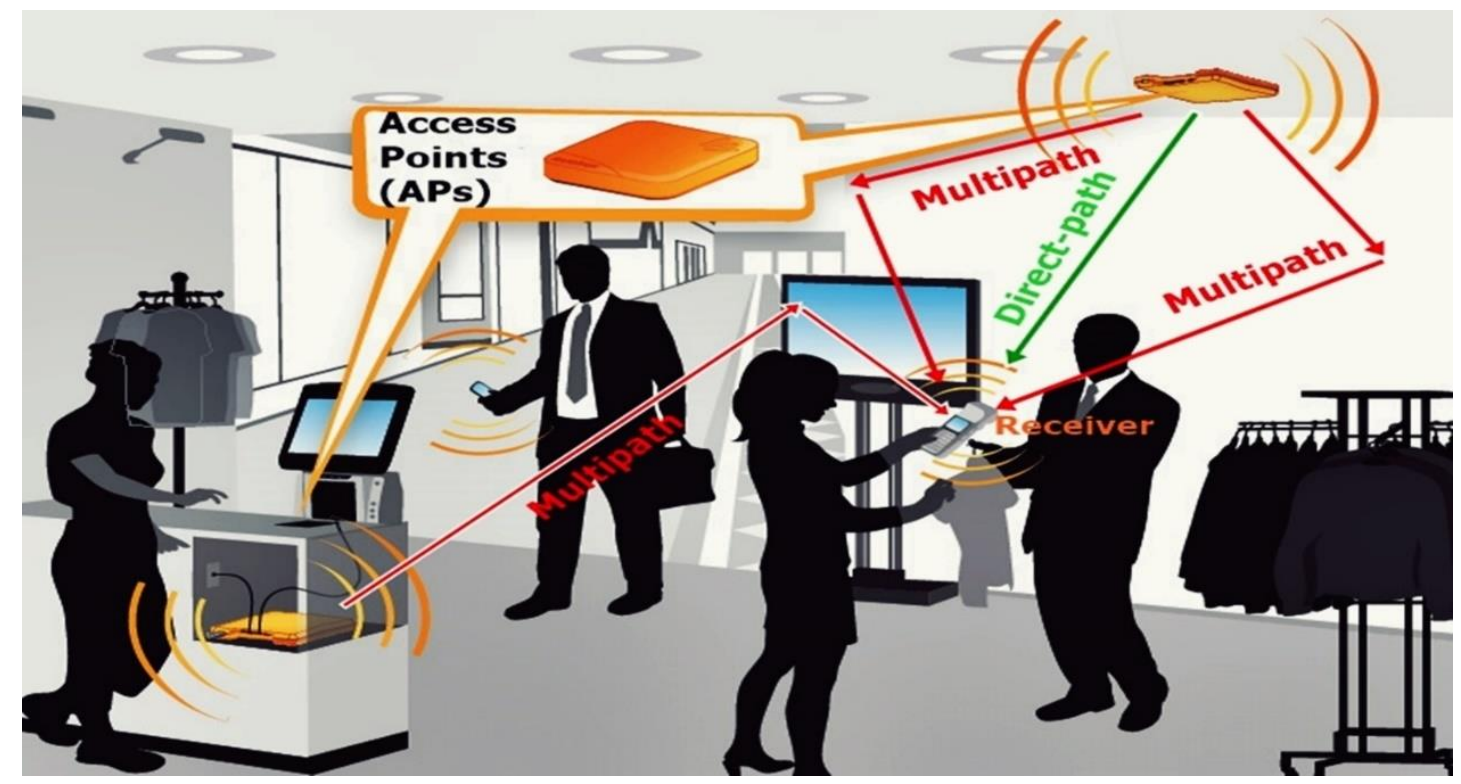

Figure 4.1: Illustration of multipath effect in an indoor environment

the characteristics of the wireless channel [64]. Wireless signals are not confined to propagate through a single path; rather the signals are emitted in the space by a transmitter. As a result, the natural deterioration in the signal's power, termed as free space loss, which occurs due to propagation in the free space is not the only factor that affects the received signal. Along with free space loss, multipath signals play a vital role in degrading signal quality at the receiver's end. A multipath signal is defined as the received signal that is reflected, diffracted or scattered by the existing obstacles in the environment after it was transmitted from the origin (i.e. transmitter). Also, a multipath signal arrives at the end point either distorted (in amplitude or phase), delayed or both.

In addition to that, distortions in the received signal will change over time because the surrounding environment is not stationary. With all the processes happening simultaneously, the received signal will seem to be subjected to random fadings. Rayleigh and Rician are the two common distributions that are used to represent the random amplitude fading. Rayleigh distribution is used in a Non-line-of-sight (NLOS) environment when the received signal is composed of only multipath signals, but no direct path signal. On the contrary, in a Line-of-sight (LOS) environment, where a dominant signal is received along with scattered multipath signals, fading is modeled with a Rician distribution. Figure 4.1 depicts a typical multipath scenario in an indoor environment, which shows a possibility of generating several multipath signals for every transmitted signal. 
To represent the statistics of RSS observables, consider a wireless channel between two nodes $p$ and $q$ in a shadowing multipath environment. The multipath environment is described by its time-varying impulse response, which is [3]

$$
h_{p q}(t ; \tau)=\sum_{n=1}^{N(t)} a_{n}(t) e^{j \phi_{n}(t)} \delta\left[\tau-\tau_{n}(t)\right]
$$

where

$$
\begin{aligned}
a_{n}(t) & =\prod_{m=1}^{M_{n}(t)} a_{m n}(t) \\
\phi_{n}(t) & =\sum_{m=1}^{M_{n}(t)} \phi_{m n}(t) \\
\tau_{n}(t) & =\sum_{m=1}^{M_{n}(t)} \tau_{m n}(t)
\end{aligned}
$$

and $a_{n}(t), \phi_{n}(t)$, and $\tau_{n}(t)$ represent the amplitude, phase and time-delay of the $n$-th impulse, respectively. It is assumed that the signal is wideband such that multipaths arriving at different time-delays, $\tau_{n}(t)$, are separable. Furthermore, $a_{m n}(t), \phi_{m n}(t)$, $\tau_{m n}(t)$ denote the time-varying attenuation, phase-change, and time delay at time $t$ due to LOS propagation path between the $(m-1)$-th and $m$-th of $M_{n}$ scatterers along the $n$-th propagation path. Also, $N(t)$ is the total number of received multipath signals at time $t$, $\tau_{n}(t)$ is the time delay associated with the $n$-th propagation path, and $h_{p q}(t ; \tau)$ is timevarying impulse response of the channel between nodes $p$ and $q$ at time $t$ to a unit impulse $\delta[$.$] transmitted at time t-\tau$.

Let the set of recorded RSS observables from an AP (for known path loss exponent and fixed transmitter-receiver radial separation) be denoted by $z$. Assuming $s_{p q}$ is the transmitted signal and $v_{p q}$ is the induced voltage across terminals of the antenna on the receiver side, $v_{p q}$ can be expressed as [3] 


$$
\begin{aligned}
v_{p q} & =s_{p q}(t) * h_{p q}(t ; \tau) \\
& =s_{p q}\left(\tau_{n}\right) a_{n}\left(\tau_{n}\right) e^{j \phi_{n}\left(\tau_{n}\right)}
\end{aligned}
$$

Considering central limit theorem, it can be seen from (4.3) that $v_{p q}$ is observed to be lognormally distributed [3] and so RSS observables $\left(z(\right.$ in $\left.\mathrm{dB}) \propto \log \left|v_{p q}\right|^{2}\right)$ follow a Gaussian distribution [3], i.e.

$$
Z \sim \mathcal{N}\left(\mu, \sigma_{R S S}\right)
$$

where $\mu$ is the mean RSS in $\mathrm{dB}$, and $\sigma_{R S S}$ is the standard deviation in $\mathrm{dB}$, which does not depend on the distance between transmitting and receiving devices and/or the transmitted power; and only subjects to the multipath environment. In most indoor surroundings, $\sigma_{R S S}$ varies from 2 to $6 \mathrm{~dB}$ [22]. To illustrate, the distribution of RSS measurements inside ICT building on the University of Calgary campus is shown in Figure 4.2. The figure demonstrates that for a fixed transmitter-receiver radial separation, the obtained RSS from an AP follows a Gaussian distribution with $\mu=46 \mathrm{dBf}$ and $\sigma_{R S S}=6 \mathrm{dBf}$.

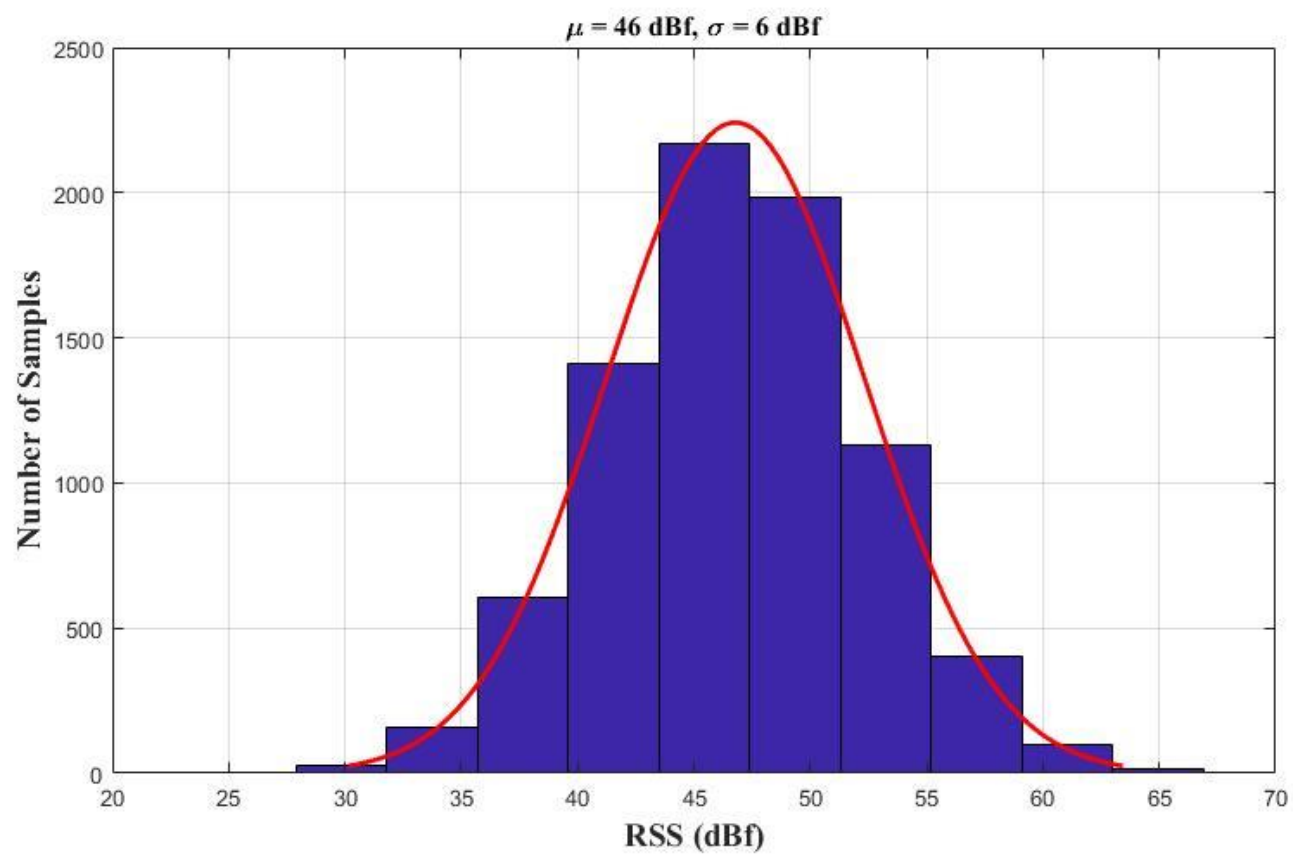

Figure 4.2: Distribution of RSS measurements form an AP with fixed transmitterreceiver radial separation inside ICT Building 


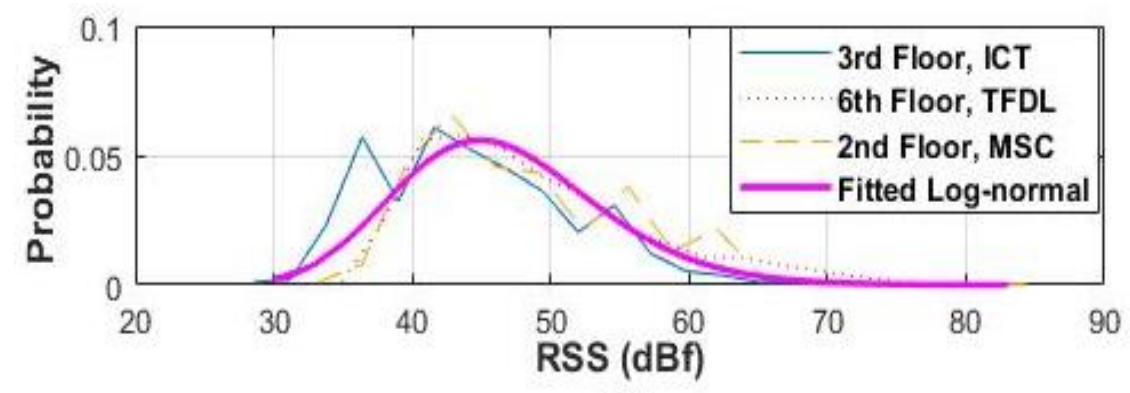

(a)

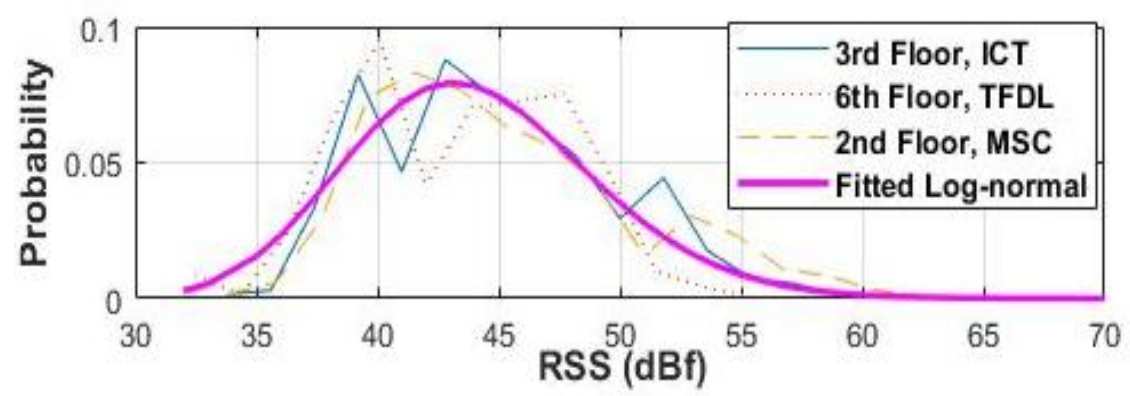

(b)

Figure 4.3: (a) Distribution of RSS ( $\left.\left|t_{m}-\ell_{f}\right|=0\right)$, (b) Distribution of RSS $\left(\left|t_{m}-\ell_{f}\right|=1\right)$

In indoors, when a Wi-Fi network scan is performed while roaming on a floor, multiple signals are received from a plurality of APs. As shown in Figure 4.2, RSS from an AP with fixed transmitter-receiver radial separation follows a Gaussian distribution. Hence, when multiple APs are visible to a receiver device (e.g. a smartphone), the RSS observables corresponding to those APs form a multimodal mixture of Gaussians. The conducted measurements show that this multimodal mixture of Gaussians can be closely approximated by a log-normal density for the variety of buildings considered in this dissertation (for more details about the buildings see Section 3.4).

Let $p\left(\mathcal{D} \mid \ell_{f}\right)$ denote the distribution of observed data $\mathcal{D}$ for given floor hypothesis $\ell_{f} \in \mathcal{L}\left(\ell_{f}\right.$ was defined in Sub-section 3.3.1). The observed data $\mathcal{D}=\left\{d_{1}, \ldots, d_{M}\right\}$ such that $d_{m}:=\left\{z_{m}, t_{m}\right\}$ is a pair-set with $z_{m}$ denoting the $m$-th measured RSS value (in dBf) and $t_{m}$ denotes the floor location of the corresponding AP transmitting the signal. Figure 4.3 shows histograms for three arbitrarily selected combinations of AP and mobile floor locations and buildings. The fitted log-normal density is clearly a good approximation for $p\left(z_{m} \mid \ell_{f}\right)$ and is supported by the rest of the measurements. 
TABLE 4.1

STATISTICAL CHARACTERISTICS OF LOG-NORMAL DISTRIBUTION

\begin{tabular}{|c|c|c|c|c|c|}
\hline & $\mathcal{F}$ & ICT & MSC & TFDL & Average \\
\hline \multirow{3}{*}{$E\left[D_{\mathcal{F}}\right](\mathrm{dBf})$} & 0 & 46 & 46 & 46 & 46 \\
\hline & 1 & 42 & 43 & 40 & 42 \\
\hline & $>1$ & 38 & 39 & - & 39 \\
\hline \multirow{3}{*}{$\operatorname{std}\left[D_{\mathcal{F}}\right](\mathrm{dBf})$} & 0 & 6 & 7 & 7 & 7 \\
\hline & 1 & 4 & 5 & 3 & 4 \\
\hline & $>1$ & 2 & 3 & - & 3 \\
\hline \multirow{3}{*}{ range $\left[z_{\mathcal{F}}\right]$} & 0 & {$[30,71]$} & {$[31,77]$} & {$[31,74]$} & {$[31,74]$} \\
\hline & 1 & {$[32,53]$} & {$[32,60]$} & {$[33,47]$} & {$[32,53]$} \\
\hline & $>1$ & {$[34,39]$} & {$[33,46]$} & - & {$[34,43]$} \\
\hline \multirow{3}{*}{$\phi_{\mathcal{F}}$} & 0 & $73 \%$ & $53 \%$ & $89 \%$ & $73 \%$ \\
\hline & 1 & $27 \%$ & $36 \%$ & $11 \%$ & $25 \%$ \\
\hline & $>1$ & $0 \%$ & $11 \%$ & 0 & $2 \%$ \\
\hline
\end{tabular}

Let $\mathcal{D}_{\mathcal{F}}$ denote the observed data for a given mobile-AP floor separation $\mathcal{F}=\mid t_{m}-$ $\ell_{f} \mid$. TABLE 4.1 shows that, regardless of building and mobile/AP floor location, the measured mean and standard deviation are highly correlated with $\mathcal{F}$. TABLE 4.1 summarizes the estimated mean and standard deviation corresponding to best-fit lognormal densities as a function of $\mathcal{F}$. Appendix B provides detail lists of measured mean standard deviation as a function of $\mathcal{F}$ corresponding to all selected buildings. TABLE 4.1 also shows the percentage of discovered APs, denoted as $\phi_{\mathcal{F}}$ for $\mathcal{F}=0,1,2$ for each of the three buildings considered in this research. Note that the majority of discovered APs $(\sim 73 \%)$ belong to the category of $\mathcal{F}=0$, some $(\sim 25 \%)$ to $\mathcal{F}=1$ and only about $\sim 2 \%$ to $\mathcal{F}>1$. As can be seen, because of the building materials and structures, no signals were 


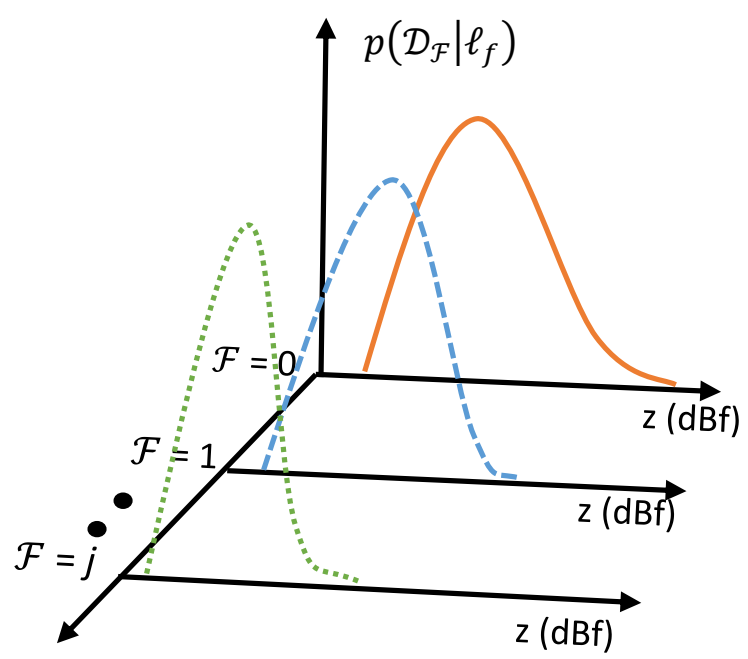

Figure 4.4: Conditional probability density $p\left(\mathcal{D}_{\mathcal{F}} \mid \ell_{f}\right)$

received in TFDL when $\mathcal{F}>1$. The $p\left(D \mid \ell_{f}\right)$ is a mixture of conditional probability density functions $p\left(\mathcal{D}_{\mathcal{F}} \mid \ell_{f}\right)$ and $2 \mathrm{D}$ distribution of $p\left(D \mid \ell_{f}\right)$ can be expressed as

$$
p\left(\mathcal{D} \mid \ell_{f}\right)=\left\{\begin{array}{cc}
\phi_{0} p\left(\mathcal{D}_{0} \mid \ell_{f}\right) & \mathcal{F}=0 \\
\vdots & \vdots \\
\phi_{j} p\left(\mathcal{D}_{j} \mid \ell_{f}\right) & \mathcal{F}=j
\end{array}, \quad \forall \ell_{f} \in \mathcal{L}\right.
$$

where $j$ is the maximum $\mathcal{F}$ observed from a Wi-Fi scan. Figure 4.4 shows $p\left(\mathcal{D} \mid \ell_{f}\right)$ as a mixture of $p\left(\mathcal{D}_{\mathcal{F}} \mid \ell_{f}\right)$ for a given $\ell_{f}$ and $\mathcal{F}$. From the analysis, it was observed that the proposed floor localization algorithm is not sensitive to the exact value of $\phi_{\mathcal{F}}$ as long as the value of $\phi_{\mathcal{F}=0}$ is larger than that for $\mathcal{F}>0$.

\subsection{Conventional RSS-based Floor Localization Methods}

As mentioned in Section 2.2, fingerprinting approach and multilateration models are two well-known methods for indoor localization using RSS. In addition to that, maximum likelihood (ML) framework has become a popular approach for floor localization [29, 30, $31,32,22]$. In this section, these conventional methods will be briefly described. 


\subsubsection{RSS Fingerprinting}

RSS fingerprinting is based on the concept that signal strength changes over different places, which in turn, makes it possible to assign each selected location with a unique signal signature (termed as fingerprint) that can assist to identify that location with unknown coordinates. Accessibility to large number of unique fingerprints leads to better location accuracy. The fingerprinting technique can be divided into two stages: 1) offline or training phase, and 2) online or localization phase [25, 63]. During the training phase, network administrators generate a database with RSS values at some reference locations inside a building. During the online phase, RSS readings captured by a mobile device (e.g. smartphone) is compared with the pre-stored values in the database to locate the device. Let, during the training phase, $Z^{T P}{ }_{i}=\left\{Z^{T P}{ }_{1}, Z^{T P}{ }_{2} \ldots, z^{T P}{ }_{m}\right\}$ be the set of RSS observables of length $m$ at the $i$-th location, whose associated coordinates $\left(x_{i}, y_{i}, z_{i}\right)$ are known, and $z^{T P}{ }_{m}$ denotes the RSS obtained from $m$-th AP. The set that contains information, such as $Z^{T P}$, IDs of the observed APs, coordinates of location $i$ etc., is called a fingerprint. The generated database, termed as radio map, combines all such fingerprints collected at different reference locations.

Let $Z^{O P}=\left\{Z_{1}^{O P}, z_{2}{ }_{2} \ldots, Z^{O P}{ }_{m}\right\}$ represent the RSS set observed during the online phase after a network scan is performed at an unknown position with coordinates $c=$ $\left(x_{u}, y_{u}, z_{u}\right) \cdot z_{m}^{O P}$ is the observed RSS value from the $m$-th AP. To identify the location, a pattern matching method, such as KNN (mentioned earlier in Section 2.2), is utilized. As an example, 1-nearest neighbor (1-NN) aims to locate the user's unknown position as [25]

$$
\hat{c}=\operatorname{argmin}\left(d\left[Z^{O P}, Z^{T P}{ }_{i}\right]\right) ; i=1,2,3, \ldots, N_{f}
$$

where $\hat{c}$ is the estimation of the unknown position, $d[$.$] denotes the dissimilarity, and N_{f}$ is the total number of fingerprints in the database. Further, $d\left[Z^{O P}, Z^{T P}{ }_{i}\right]$ can be shown as the squared Euclidean distance between $Z^{O P}$ and $Z^{T P}{ }_{i}$ [25]. i.e.

$$
d\left[Z^{O P}, Z^{T P}{ }_{i}\right]=\left\|Z^{O P}-Z^{T P}{ }_{i}\right\|^{2} ; i=1,2,3, \ldots, N_{f}
$$

As mentioned in Chapter 2, to reduce the complexity and space needed in the traditional fingerprinting method, a few simplified forms of the traditional methods were 


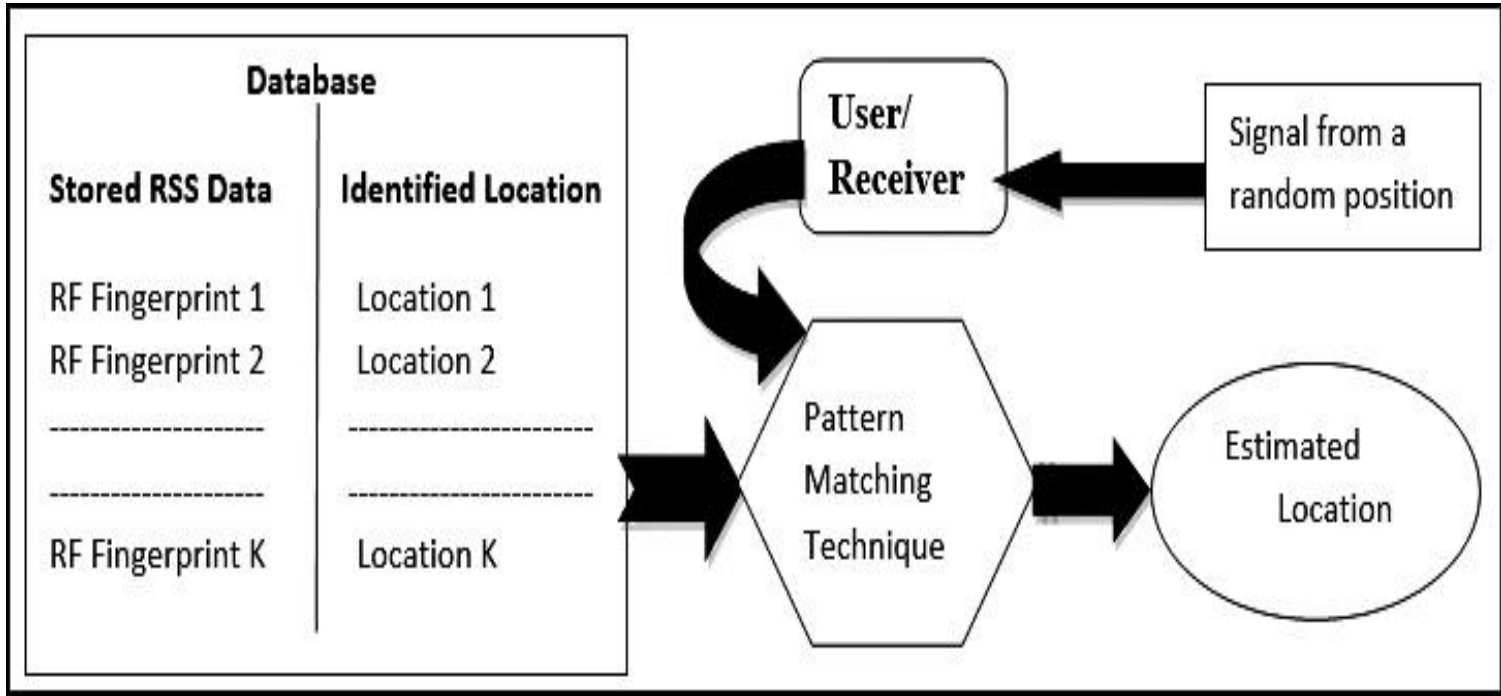

Figure 4.5: Fingerprinting approach (online phase)

proposed in the literature. For example, [20] considered only to store floor location of the APs, and [26] only considered the APs which were visible from multiple floors. The fingerprinting approach (online phase only) is summarized in Figure 4.5.

\subsubsection{RSS-based Multilateration}

Statistics-based path loss approach has become a preferable alternative in RSS-based positioning to overcome the need of storing data in large-scale and avoiding data transmission bottlenecks. As observed RSS over a wireless channel is unpredictable and non-stationary, a statistical model is followed to estimate the distance between transmitter and receiver. Subsequently, a multilateration technique is implemented to determine the smartphone user's unknown location. For instance, the trilateration technique uses distances between the receiver (i.e. smartphone) and 3 APs to determine user location on a 2-dimensional (2-D) plane. Three distances are used as radii to form three circles with the transmitters being at the center. Later, the point where the three circles intersect is selected as the user location.

Although path loss approach does not require storing a large set of data, it is essential to have knowledge about a few parameters related to the environment where the positioning system is implemented. In traditional path loss approach, coordinates of the APs and their transmitted power along with path loss coefficient are considered as the required parameter 
for positioning [25]. Moreover, the concept of free space wave propagation is exploited, which provides with the following equation

$$
P_{r_{i}}=P_{T_{j}}-10 \eta \log _{10} d_{j, i}+n_{j, i}
$$

where $P_{r_{i}}$ is the received signal strength at $i$-th measurement point (user location), $P_{T_{j}}$ is the transmitted power by $j$-th AP, $\eta$ is the path loss coefficient ( $\eta$ varies from 2 to 5 [63]), and $n_{j, i} \sim \mathcal{N}\left(0, \sigma_{n}{ }^{2}\right)$ is a noise factor due to measurement errors, small-scale fading, and shadowing. Lastly, $d_{j, i}$ is the Euclidean distance between $j$-th AP and $i$-th measurement point, which can be expressed as

$$
d_{j, i}=\sqrt{\left(x_{i}-x_{j}\right)^{2}+\left(y_{i}-y_{j}\right)^{2}+\left(z_{i}-z_{j}\right)^{2}}
$$

where $\left(x_{i}, y_{i}, z_{i}\right)$ and $\left(x_{j}, y_{j}, z_{j}\right)$ are the coordinates of the $i$-th measurement point and $j$-th AP, respectively.

\subsubsection{Maximum Likelihood-based Approach}

Due to its asymptotic optimality, ML is an attractive framework applicable to a variety of problems involving parameter estimation and classification. Authors in [29, 30, 31, 32, 22] applied ML framework to floor localization. Nevertheless, ML estimators and classifiers are inherently biased and are prone to significant issues due to the presence of singularities when applied to problems involving multi-modal Gaussian distribution mixtures such as Wi-Fi RSS observables inside buildings.

Approaches in this category only require the address of the APs and their locations, and no offline calibration is needed. During the online phase, different algorithms are followed to determine user location. Two of such algorithms are [22]:

a) Maximum RSS: After a Wi-Fi scan is performed during localization phase, maximum RSS approach detects the AP from which maximum RSS is observed and selects the corresponding floor hypothesis $\ell_{f}$ as the output of the algorithm, i.e.

$$
\ell_{M L}=\operatorname{argmax}\left(Z^{O P}\right)
$$


where $\ell_{M L}$ is the floor selected as the output and $Z^{O P}$ as defined earlier is the set of RSS observables during localization phase. Maximum RSS approach is a simplified form of sum-RSS algorithm (see Section 2.2 for more details), where the summation of RSS (observed during the scan) associated with each floor is calculated and the floor with maximum summation value is given as the output of the algorithm.

b) Maximum Number of Available APs: In this approach, the system scans the WiFi network and the floor hypothesis $\ell_{f}$ associated with the largest number of visible access points is selected as the output of the detection algorithm, i.e.

$$
\ell_{M L}=\operatorname{argmax}\left(N^{A P}\right)
$$

where $N^{A P}=\left\{n_{1}^{A P}, n_{2}^{A P}, \ldots, n_{N}^{A P}\right\}$ and $n_{i}^{A P}$, where $i=1,2, \ldots, N$, represents the number of observed APs from $i$-th floor during a network scan.

\subsection{Proposed MCBI Framework for RSS-based Floor Localization}

Both RSS fingerprinting and RSS-based multilateration approaches described in the previous section require a large number of information to be stored for floor localization [25]. Whereas, as mentioned earlier, ML-based approaches are prone to singularities. In this section, a Monte Carlo Bayesian Inference algorithm is formulated for robust RSSbased floor localization that requires less space to store information. The MCBI framework is reasonable since user floor location is typical of a discrete random variable. As will be shown shortly, in the context of RSS-based floor localization MCBI framework realizes a maximum posteriori probability (MAP) classifier. Let $p\left(\ell_{f} \mid \mathcal{D}\right)$ denote the posterior distribution of smartphone floor location $\ell_{f}$, given observed data $\mathcal{D}$ and $\ell_{f} \in \mathcal{L}$. The goal is to find the floor location $\ell_{M A P}^{R} \in \mathcal{L}$, that maximizes the posterior $p\left(\ell_{f} \mid \mathcal{D}\right)$ for a given prior $p\left(\ell_{f}\right)$ and data $\mathcal{D}$. As in many problems of practical interest, however, exact inference based on analytical evaluation of $p\left(\ell_{f} \mid \mathcal{D}\right)$ from prior $p\left(\ell_{f}\right)$ and data $\mathcal{D}$ is rather complex. Fortunately, an alternative class of algorithms based on Monte Carlo approximation provide powerful framework for the application of Bayesian Inference to everyday problems [65]. In this dissertation, a category of MCBI methods known as 'samplingimportance-resampling' is explored. While the accuracy of Monte Carlo approximation 
improves when a larger number of independent samples are used, nevertheless, the overall accuracy does not depend on the dimensionality of the sampling space, and high accuracy may be achieved with relatively small number of samples $[65,66]$.

The first step is to draw unweighted samples from the prior $p\left(\ell_{f}\right)$. When no prior information about smartphone user floor location is available, sampling from a noninformative prior (uniform distribution) is an obvious strategy. For example, for floor localization in an $N$-story building $N$ unweighted samples of a uniform prior distribution can be obtained such that $p\left(\ell_{f}\right)=1 / N \forall \ell_{f} \in \mathcal{L}$. However, a more effective strategy is to draw samples from areas of $p\left(\ell_{f}\right)$ with high probability. The main advantage of drawing samples from areas with high probability is that very efficient posterior inference can result from a small number of samples $[65,66]$. Let $\ell_{f} \in \mathcal{L}^{s}$ denote samples drawn from areas of the prior distribution with high probability such that

$$
p\left(\ell_{f}\right)=\frac{1}{N_{\mathrm{s}}}, \quad \mathcal{L}^{s} \subseteq \mathcal{L} \text { and } N_{\mathrm{s}} \leq N
$$

In this dissertation, the sample set $\mathcal{L}^{s}$ includes the subset of floors that are associated with the APs discovered during a recent wireless network scan. This is feasible since due to RF pathloss and penetration losses in buildings, APs with large smartphone-AP floor separation $(>2)$ are highly unlikely to be discovered, as shown in TABLE 4.1.

The next step is to generate a set of posterior samples by updating the prior samples through the medium of importance weighting. Recall from Bayes' theorem that $p\left(\ell_{f} \mid \mathcal{D}\right)=$ $p\left(\mathcal{D} \mid \ell_{f}\right) p\left(\ell_{f}\right) / p(\mathcal{D})$ [67]. Let $\tilde{p}\left(\ell_{f} \mid \mathcal{D}\right)=p\left(\mathcal{D} \mid \ell_{f}\right) p\left(\ell_{f}\right)$ denote the unnormalized target posterior distribution. Consequently, normalized importance weights, $\omega_{f}$, may be calculated as [65]

$$
\omega_{f}=\frac{\tilde{p}\left(\ell_{f} \mid \mathcal{D}\right) / p\left(\ell_{f}\right)}{\sum_{\ell_{f}^{\prime}} \tilde{p}\left(\hat{\ell}_{f}^{\prime} \mid \mathcal{D}\right) / p\left(\hat{\ell}_{f}\right)}=\frac{p\left(\mathcal{D} \mid \ell_{f}\right)}{\sum_{\ell_{f}^{\prime}} p\left(\mathcal{D} \mid \hat{\ell}_{f}\right)}, \quad \ell_{f} \in \mathcal{L}^{\mathrm{s}}
$$

where the quantity $p\left(\mathcal{D} \mid \ell_{f}\right)$ is evaluated for the observed dataset $\mathcal{D}$. Since RSS observables from spatially separated APs are statistically independent due to multipath fading inside buildings, $p\left(\mathcal{D} \mid \ell_{f}\right)$ can be calculated from the product rule of probability as 


$$
p\left(\mathcal{D} \mid \ell_{f}\right)=\prod_{m} p\left(d_{m} \mid \ell_{f}\right), \quad \ell_{f} \in \mathcal{L}^{\mathrm{s}}
$$

where $p\left(d_{m} \mid \ell_{f}\right)$ is calculated using normalized conditional probability density $p\left(\mathcal{D}_{\mathcal{F}} \mid \ell_{f}\right)$ introduced in Section 4.2.

The calculated weights $\omega_{f}$ from (4.13) are then used to approximate the posterior probabilities as

$$
p\left(\ell_{f} \mid \mathcal{D}\right)=\omega_{f}, \quad \ell_{f} \in \mathcal{L}^{\mathrm{s}}
$$

Finally, a MAP classifier based on maximum posterior expectation picks mobile user floor location as

$$
\ell_{\mathrm{MAP}}^{R}=\arg \max _{\ell_{f} \in \mathcal{L}^{\mathrm{s}}}\left[p\left(\ell_{f} \mid \mathcal{D}\right)\right]
$$

\subsection{Results and Analysis}

To do a comprehensive performance analysis of the proposed MAP classifier, RSS readings from APs were measured alongside BP from the three multistory buildings mentioned in Section 3.4. Different structural designs and construction materials of the buildings made those well-fitted for evaluating the robustness of a localization method. Depending on the floor area to be covered, each floor in all three buildings is equipped with 5-14 APs. Nevertheless, in some areas such as washrooms, stairways, either the RSS was weak or no access to Wi-Fi was available.

As mentioned in Section 3.4, an Arduino comprising of Broadcom BCM43362 radio capable of measuring Wi-Fi RSS values with a resolution of $1 \mathrm{dBm}$ was used with a sample rate of 1 hertz for RSS measurements. Data $\mathcal{D}$, comprising of $2.4 \mathrm{GHz}$ Wi-Fi RSS from a plurality of APs and corresponding floor locations was captured, as the mobile device was moved by a pedestrian. For each mobile floor location, $\ell_{f}$, measured RSS values $z$, were grouped based on their corresponding AP floor locations $t$. It is required to store the address of the APs and their corresponding floor numbers in the database. The information about the APs inside a building can be easily obtained from the network administrator of that 


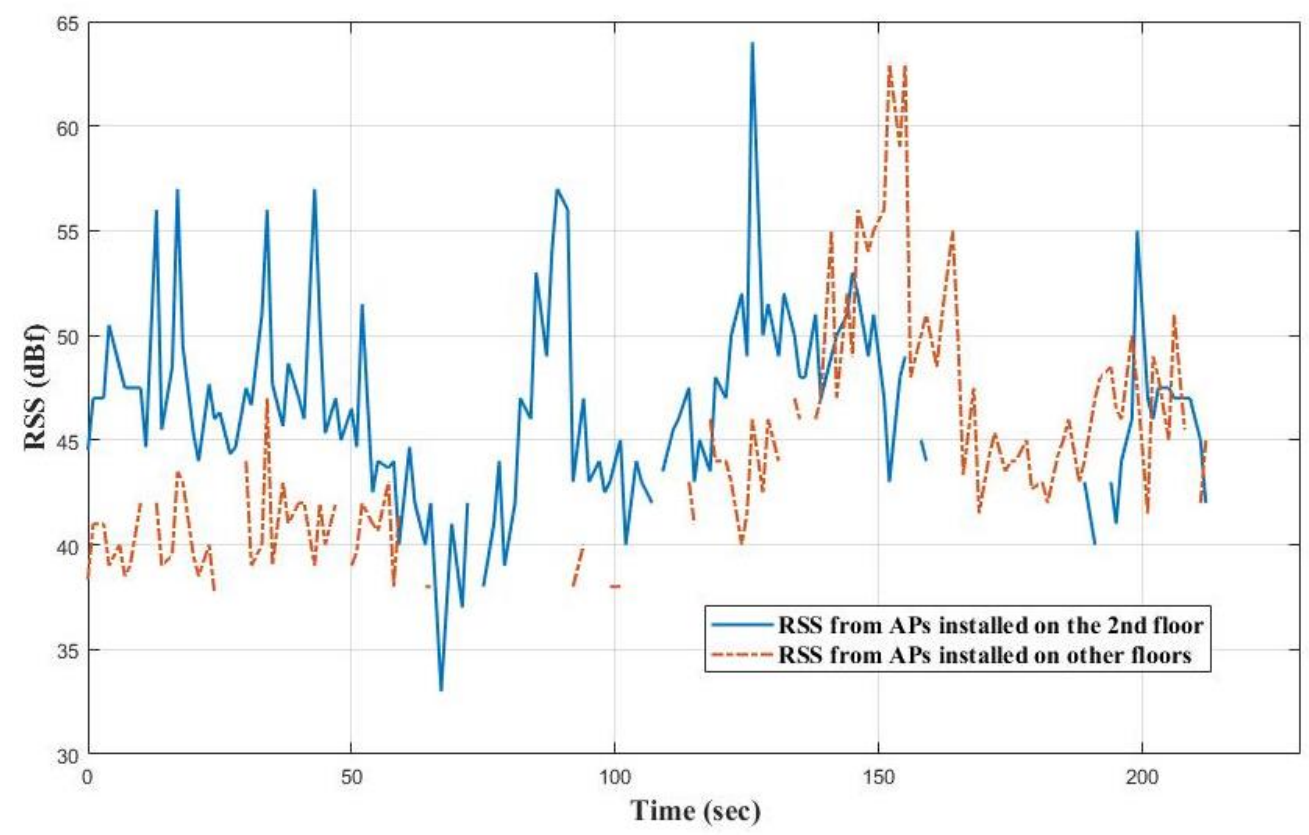

Figure 4.6: RSS map (ICT, 2nd Floor)

building. For this dissertation, initially the required information related to APs was obtained through site surveys, and the process is shown in APPENDIX C. Subsequently, the information was verified by contacting The Information Technologies (IT) department of the University of Calgary.

The analysis started with the observation of signal propagation, which can have an impact on floor localization. Figure 4.6 illustrates an instance where measurement was conducted on the second floor of ICT building. The figure shows that signals were received from APs that are located on the second floor as well as other floors. In addition to that, during the measurements, at some points signals were only observed from APs located on other floors due to multipath propagation and surrounding obstacles. As such, RSS-based approaches would provide inaccurate floor localization. Note that the achievable performance is also influenced by the availability of APs on each floor and increasing the number of APs can improve the floor localization accuracy [68]. Figure 4.7 demonstrates the impact of AP availability on floor localization accuracy when RSS-based MAP classifier was applied for the measurements performed inside ICT building. The placements of the APs on all floors were randomly selected over 100 realizations. As can 


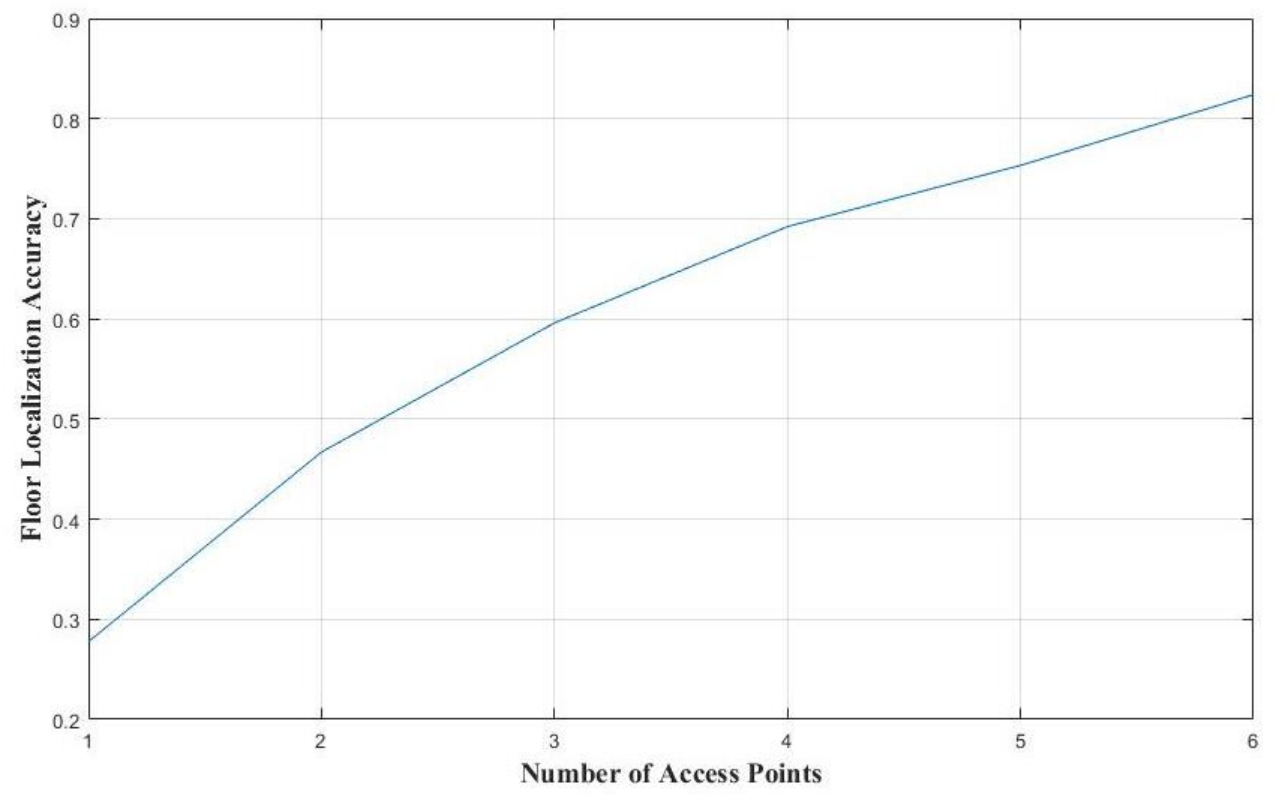

Figure 4.7: Impact of AP availability on floor localization

be seen from Figure 4.7, increment in the number of APs improves the performance of floor localization.

TABLE 4.2 shows RSS-based floor localization accuracy based on the proposed MCBI framework introduced in Section 4.4. To investigate the sensitivity of the RSS-based MAP classifier to the choice of measured RSS distribution $p\left(D_{\mathcal{F}} \mid \ell_{f}\right)$, which may vary from one building to another, a more computationally efficient uniform distribution was also incorporated and tested against the carefully measured log-normal distribution shown in

TABLE 4.2

FLOOR LOCALIZATION ACCURACY USING RSS-BASED ALGORITHMS

\begin{tabular}{|c|c|c|c|c|}
\hline & ICT & TFDL & MSC & Average \\
\hline MAP (log-normal) & $91 \%$ & $98 \%$ & $58 \%$ & $81 \%$ \\
\hline MAP (uniform) & $86 \%$ & $96 \%$ & $59 \%$ & $76 \%$ \\
\hline Maximum RSS & $84 \%$ & $99 \%$ & $60 \%$ & $80 \%$ \\
\hline Maximum APs & $79 \%$ & $96 \%$ & $51 \%$ & $74 \%$ \\
\hline
\end{tabular}


Figure 4.3. As can be seen from TABLE 4.2, the proposed floor localization algorithm results in $5 \%$ performance difference on average based on the choice of $p\left(D_{\mathcal{F}} \mid \ell_{f}\right)$.

Results from two prior RSS-based floor localization frameworks from [22] (described in Sub-section 4.3.3) are also included in TABLE 4.2 for comparison. As can be seen, the proposed MAP classifier with informative prior (log-normal distributions) outperforms other RSS-based floor localization methods. Also note from TABLE 4.2 that RSS-based floor localization inside MSC, which is typical of a building with irregular structure and hollow spaces, has low floor localization accuracy (e.g. 58\% when MAP classifier was applied), which is a significant limitation of RSS-based methods.

\subsection{Summary}

In this chapter, it was shown that Wi-Fi RSS distributions in an indoor environment, where signals are received from plurality of APs, can be approximated by log-normal densities. The statistical characteristics of the RSS distributions were also derived for the three-buildings considered in this dissertation and it was shown that APs with large smartphone-AP floor separation $(>2)$ are highly unlikely to be discovered.

An MCBI framework (i.e. MAP classifier) for RSS-based floor localization was formulated to compensate for the drawbacks of the conventional RSS-based floor localization methods. Over extensive measurements, the performance of the proposed floor localization algorithm was evaluated, and it was shown that the proposed MAP classifier outperforms two ML RSS-based approaches. Nevertheless, all RSS-based methods resulted in low floor localization accuracy when measurement was conducted inside MSC. As such, although RSS-based methods can be readily implemented with the exiting infrastructure, those methods are not reliable for floor localization inside a building with irregular structures and hollow interiors. 


\section{Chapter 5: Floor Localization Using Proposed Sensor Fusion Framework}

\subsection{Introduction}

The primary goal in designing floor localization system is to formulate a system at reasonable cost that has high accuracy and low complexity. As described in previous chapters, although low-cost floor localization systems based on barometric altimetry (BA) and received signal strength (RSS)-based are readily available, both systems lack the required accuracy for many commercial and non-commercial applications such as search and rescue. As well, differential barometric altimetry (DBA)-based floor localization systems, although fairly accurate, are not compatible with the available infrastructure. As such, a method is required that can be conveniently implemented to gain high accuracy in floor detection.

In this chapter, a novel RSS-aided BA algorithm is proposed for floor localization, where the novelty is the fusion (combination) of both the RSS and barometer measurement values using Kalman filter (KF). The proposed system can be deployed with available infrastructure and can achieve high accuracy. The result is comparable to that of DBAbased floor localization system. The rest of the chapter is structured as follows. In Section 5.2 a brief description about KF is given. The proposed sensor fusion algorithm is formulated in Section 5.3. Section 5.4 evaluates the performance of the sensor fusion algorithm in comparison with that of RSS-only, BA-only, and DBA-only localization methods. Lastly, a summary is given in section 5.5.

\subsection{Kalman Filter}

Kalman filter is over 50 years old, and yet it is one of the most prevalent and popular techniques for data fusion in use today. The filter was named after Rudolf Emil Kalman, who proposed it in late 1958 [69]. KF is a convenient mathematical tool to solve a problem, which follows a Bayesian approach and uses prior knowledge to estimate the next state of 


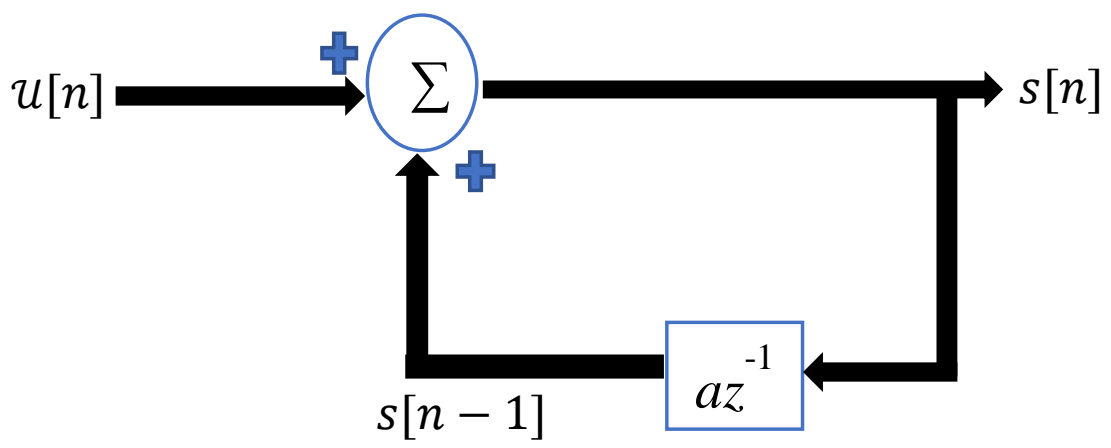

Figure 5.1: Block diagram of state model

the system. Use of prior knowledge increases the accuracy of the estimator [70]. KF has found its application in time varying channel estimation, control and navigation of vehicle, sensor fusion and so on $[69,70]$. One of the earlier famous use of $\mathrm{KF}$ is its inclusion in Apollo onboard guidance system [69].

The estimation process in $\mathrm{KF}$ is recursive in nature and it utilizes a state equation and an observation equation in its implementation. KF can be applied to estimate a scalar Gauss-Markov signal or its vector extension. Also, a scalar or vector observations is included in KF's estimation process. As the suggested floor localization method uses a scalar state - scalar observation combination, this section is going to summarize the basic equations for a KF scalar state - scalar observation scenario.

A KF random process is modelled as [70]

$$
s[n]=a s[n-1]+\mathcal{U}[n] ; n \geq 0
$$

where $s[n]$ is the variable that needs to be estimated at time step $n$, and $\mathcal{U}[n]$ is the WGN with variance $\sigma_{u}{ }^{2}$, and $a$ is a transition parameter that correlates current sample with a previous sample. $\mathcal{U}[n]$ is known as driving or excitation noise and can be considered as input to the system. The current output $s[n]$ only depends on the previous state $(s[n-1])$ of the system and the current input $\mathcal{U}[n]$. The model in (5.1) is known as state or dynamical model, which is shown in Figure 5.1.

The observations (measurements) at time $n$ can be stated as

$$
x[n]=s[n]+w[n] ; n \geq 0
$$


where $x[n]$ is the observation made, and $w[n]$ is a zero mean Gaussian noise with independent samples and with a variance $\sigma_{w}{ }^{2}$, i.e. $w[\mathrm{n}] \sim \mathcal{N}\left(0, \sigma_{w}{ }^{2}\right)$.

KF estimates $s[n]$ based on the observations $\{x[0], x[1], \ldots, x[n]\}$. The estimated $s[n]$ will be denoted by $\hat{s}[n]$ and $\hat{s}[n]$ based on the observations $\{x[0], x[1], \ldots, x[n]\}$ will be expressed as $\hat{s}[n \mid n]$. To achieve optimality, KF attempts to minimize the Bayesian minimum squared error (MSE), which is [70]

$$
E\left[(s[n]-\hat{s}[n \mid n])^{2}\right]
$$

Implementation of KF can be divided into two stages: prediction and correction. The equations for implementing KF for scalar state - scalar observation are as follows [70]:

For $n \geq 0$,

i) Prediction:

$$
\hat{s}[n \mid n-1]=a \hat{s}[n-1 \mid n-1]
$$

\section{ii) Minimum Prediction MSE:}

$$
\mathcal{M}[n \mid n-1]=a^{2} \mathcal{M}[n-1 \mid n-1]+\sigma_{u}^{2}
$$

where $\mathcal{M}[n \mid n-1]$ is the minimum MSE when $s[n]$ is estimated based on the previous measurement sample, which can be expressed as

$$
\mathcal{M}[n \mid n-1]=E\left[(s[n]-\hat{s}[n \mid n-1])^{2}\right]
$$

\section{iii) Kalman Gain:}

$$
K[n]=\frac{\mathcal{M}[n \mid n-1]}{\sigma_{w}^{2}+\mathcal{M}[n \mid n-1]}
$$

where $K[n]$ is termed as the gain factor and is less than 1 .

\section{iv) Correction:}

$$
\hat{s}[n \mid n]=\hat{s}[n \mid n-1]+K[n](x[n]-\hat{s}[n \mid n-1])
$$




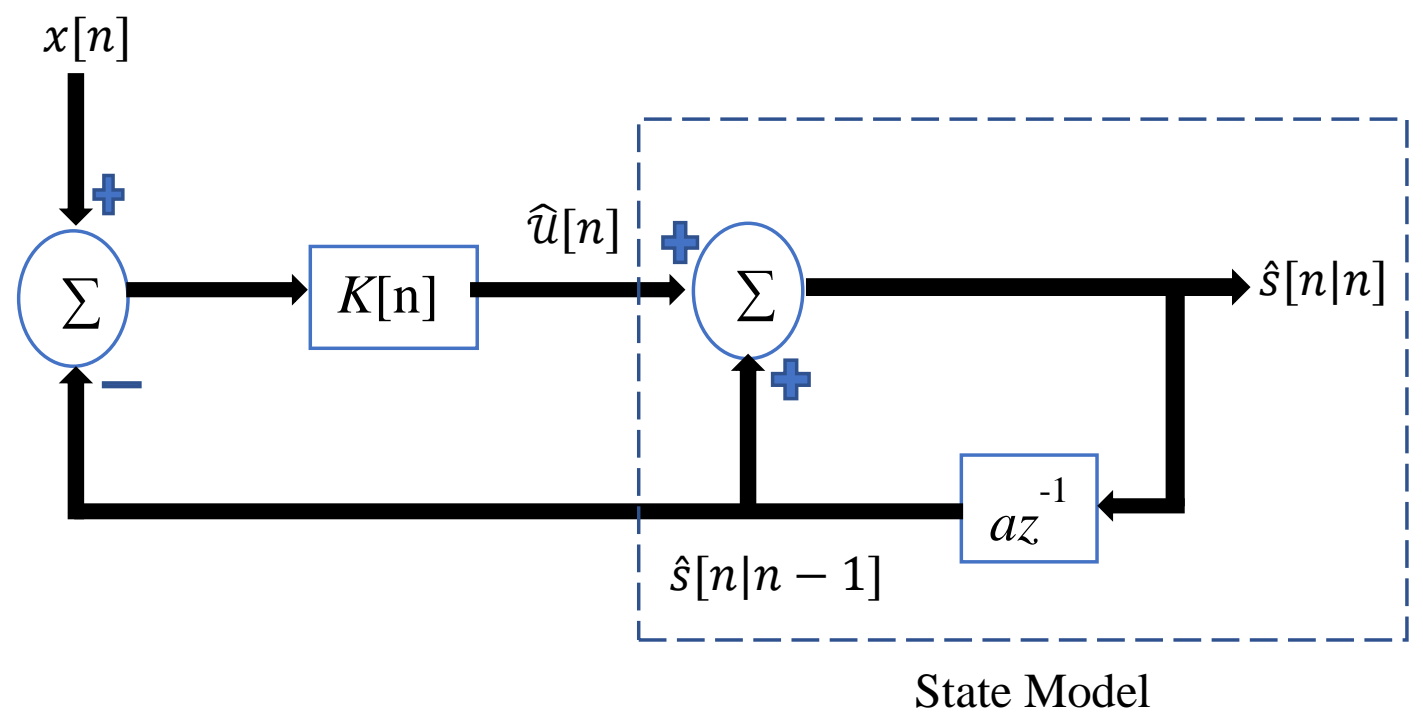

Figure 5.2: Block Diagram of the Kalman filter

v) Minimum MSE:

$$
\mathcal{M}[n \mid n]=(1-K[n]) \mathcal{M}[n \mid n-1]
$$

The first two steps mentioned above fall under the prediction stage and the last three steps are considered as the correction stage. The error increases in the prediction stage, while it decreases in the correction stage. After observing a new measurement sample, correction is made to the estimation. As $K[n]<1$, according to (5.9) the MSE decreases in the correction stage. A block diagram of KF is shown in Figure 5.2 and it can be observed that the state model shown in Figure 5.1 is an integral part of the KF. Along with that, using (5.4) and (5.8), the signal estimate can be written as,

$$
\hat{s}[n \mid n-1]=a \hat{s}[n-1 \mid n-1]+\widehat{U}[n]
$$

Hence, the output of the gain block in Figure 5.2 can be considered as $\hat{\mathcal{u}}[n]$ or estimated value of $\mathcal{U}[n]$.

\subsection{Proposed Sensor Fusion Algorithm}

Recall that the BA measurement error model, introduced in Chapter 3, is a Gaussian process. As such, KF is the optimal framework for tracking and estimating the randomly 
varying bias $b_{k}$. In the implementation stage, $\mathrm{KF}$ requires a physical model that represents how the current state of a system evolves from its previous state. In this dissertation, the first order random walk model introduced in (3.5) is used to update KF state variable during the prediction stage as

$$
b_{k+m}=b_{k}+\sqrt{m} w_{b}
$$

The correction is done when an RSS value can be mapped to a floor. In the correction stage of the KF, the Monte Carlo Bayesian Inference (MCBI) scheme developed in Chapter 4 is used to determine smartphone floor location and subsequently the associated correct floor height, denoted as $h_{R S S}$. Later, $h_{R S S}$ is used to update (correct) bias $b_{k}$. As mentioned before, the MCBI framework is reasonable since user floor location is typical of a discrete random variable. As shown in Chapter 4, in the context of RSS-based floor localization the proposed MCBI framework realizes a maximum posteriori probability (MAP) classifier. The correct floor height $h_{R S S}$ (determined from $\ell_{\mathrm{MAP}}^{R}$ using RSS-based MAP classifier) and building floor height information, which is assumed to be known a priori, are applied in the correction stage of the KF as

$$
h_{R S S}=\tilde{h}[n]-b_{k}+v[n]
$$

where $\tilde{h}[n]$ is the measured height using barometer at the $n$-th iteration of KF, $v$ is the measurement noise with standard deviation of $\sigma_{\mathrm{v}}$, and $b_{k}$ is the randomly varying bias term, defined in Chapter 3.

Finally, the last updated $b_{k}$ is used to estimate correct height according to (3.7) as

$$
\hat{h}[n] \mid b_{k}=\tilde{h}_{k+m}[n]-b_{k}
$$

where as mentioned in Section 3.3, $\hat{h}[n] \mid b_{k}$ is the estimated height for a known $b_{k}$ with $\operatorname{var}\left(\hat{h}[n] \mid b_{k}\right)=m \sigma_{b}^{2}+\sigma_{w}^{2}$. Subsequently, a MAP classifier based on minimum probability of error criteria introduced in (3.15) is used to estimate mobile user floor location as

$$
\ell_{M A P}=\underset{\ell_{f} \in \mathcal{L}}{\arg \min }\left[\left(\hat{h}[n] \mid b_{k}-\bar{h}_{f}\right)^{2}\right]
$$




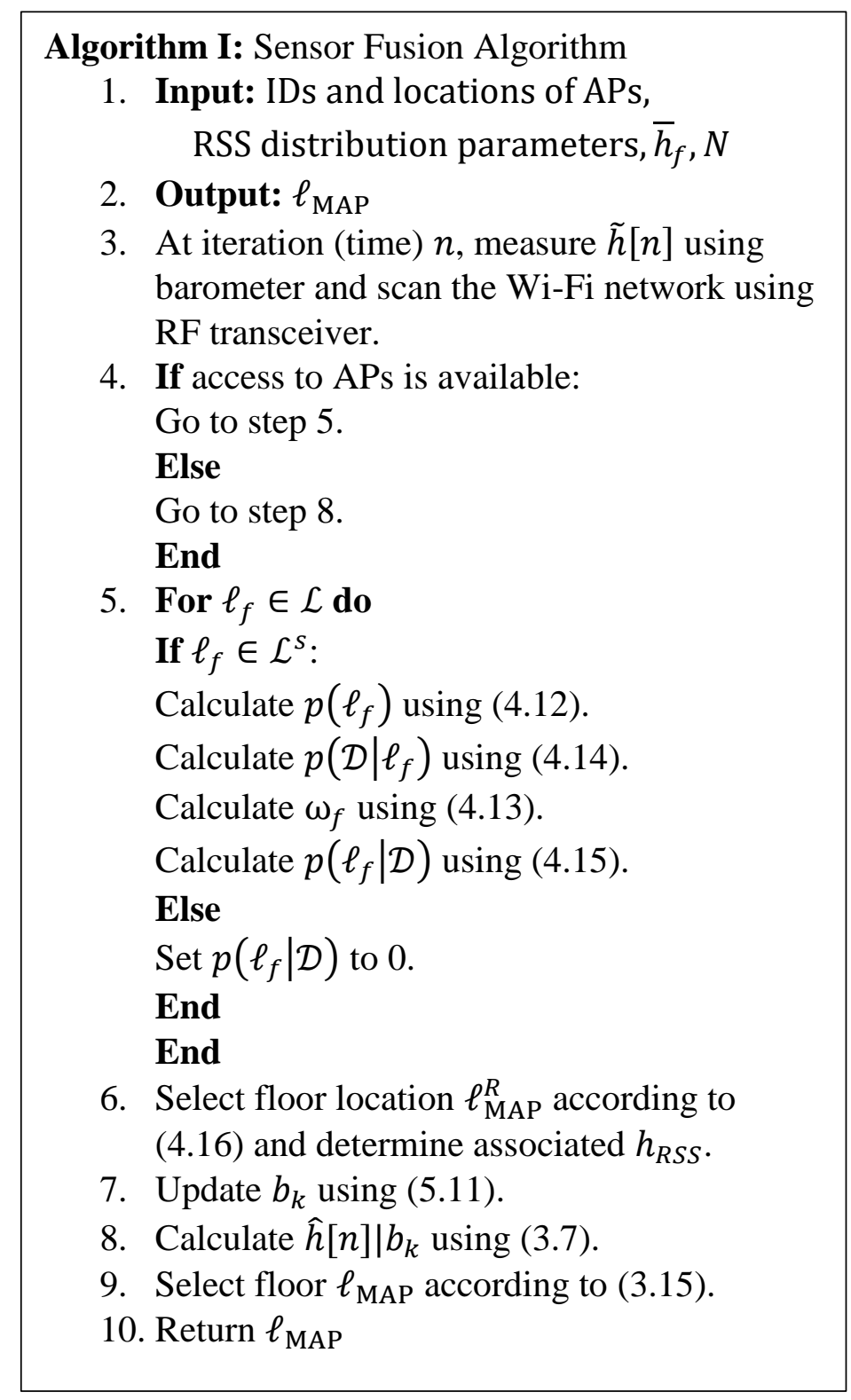

Figure 5.3: Pseudocode of sensor fusion algorithm

where $\ell_{\text {MAP }}$ is the selected floor as the output of sensor fusion algorithm and $\bar{h}_{f}$, as defined in Section 3.3, is the correct floor height associated with the floor hypothesis $\ell_{f}$, which is known a priori. A pseudocode of the proposed fusion algorithm is given in Figure 5.3. The required processing power for this fusion algorithm is compatible with smartphones of current vintage. The complexity of the algorithm in terms of number of floors $(N)$ and number of observed APs $\left(n^{A P}\right)$ after a network scan in a building is $O\left(N n^{A P}\right)$, where $O($. 
denotes the upper limit of execution time required by an algorithm. Note that for a specific building, $N$ is a constant number and as such, $O\left(N n^{A P}\right)$ is linear function in $n^{A P}$.

\subsection{Results and Analysis}

The performance of the proposed sensor fusion algorithm was evaluated over the measurements collected from all three buildings (described in Section 3.4). As mentioned in Chapter 3 and Chapter 4, both pressure and RSS readings were measured simultaneously and the proposed sensor fusion algorithm combines both information to provide enhanced floor localization.

Figure 5.4 shows estimated height $\hat{h}[n] \mid b_{k}$ using the proposed fusion algorithm for all three buildings considered in this dissertation. As can be seen from Figure 5.4, local variations of air pressure, $b_{k}$, due to the changing environmental conditions, are aptly estimated by the sensor fusion algorithm. During performed measurements, the estimated $b_{k}$ was removed from BA readings to determine correct user floor locations.

TABLE 5.1 shows floor localization accuracy for the proposed fusion algorithm. As can be seen from TABLE 5.1, the proposed fusion algorithm achieved 97\% average floor localization accuracy across all tests. Both log-normal and uniform distributions were used to approximate $p\left(D_{\mathcal{F}} \mid \ell_{f}\right)$ in MAP classifier to investigate the sensitivity of the fusion algorithm to the choice of measured RSS distribution. As can be seen from TABLE 5.1, the proposed floor localization algorithm is fairly insensitive to the choice of $p\left(D_{\mathcal{F}} \mid \ell_{f}\right)$ such that uniform and $\log$-normal distributions result in a mere $1 \%$ performance difference in floor localization.

TABLE 5.1

FLOOR LOCALIZATION ACCURACY USING SENSOR FUSION ALGORITHM

\begin{tabular}{|c|c|c|c|c|}
\hline$p\left(\mathcal{D}_{\mathcal{F}} \mid \ell_{f}\right)$ Model & ICT & TFDL & MSC & Average \\
\hline log-normal & $>99 \%$ & $>99 \%$ & $93 \%$ & $97 \%$ \\
\hline uniform & $>99 \%$ & $>99 \%$ & $92 \%$ & $96 \%$ \\
\hline
\end{tabular}




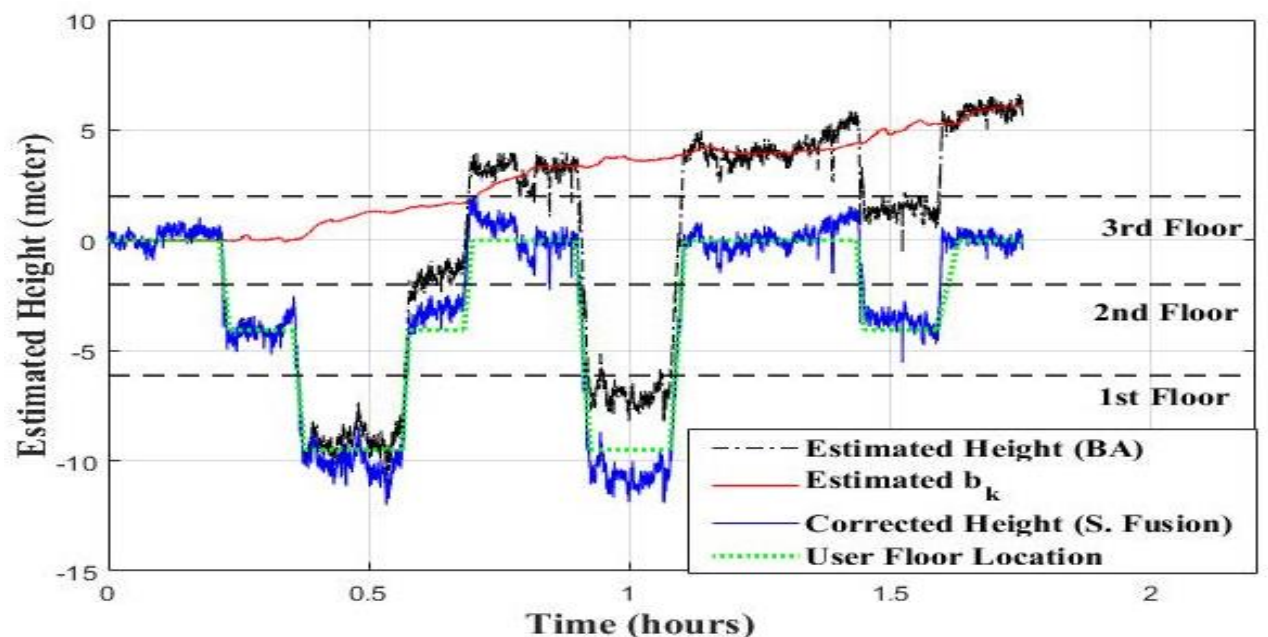

(a)

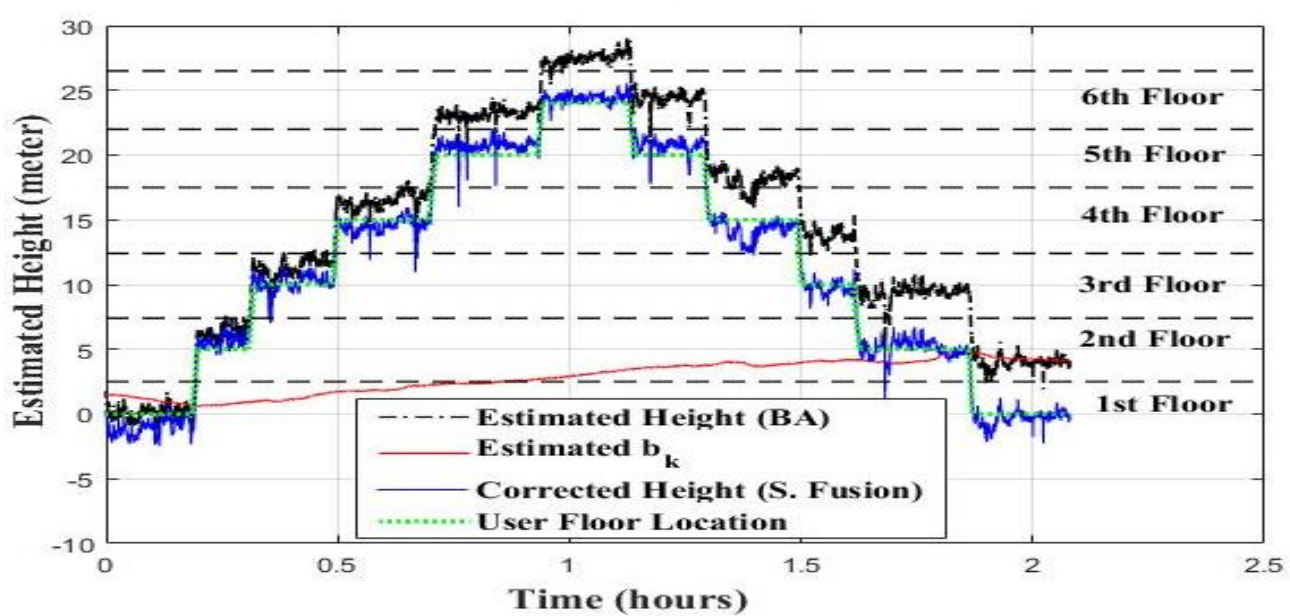

(b)

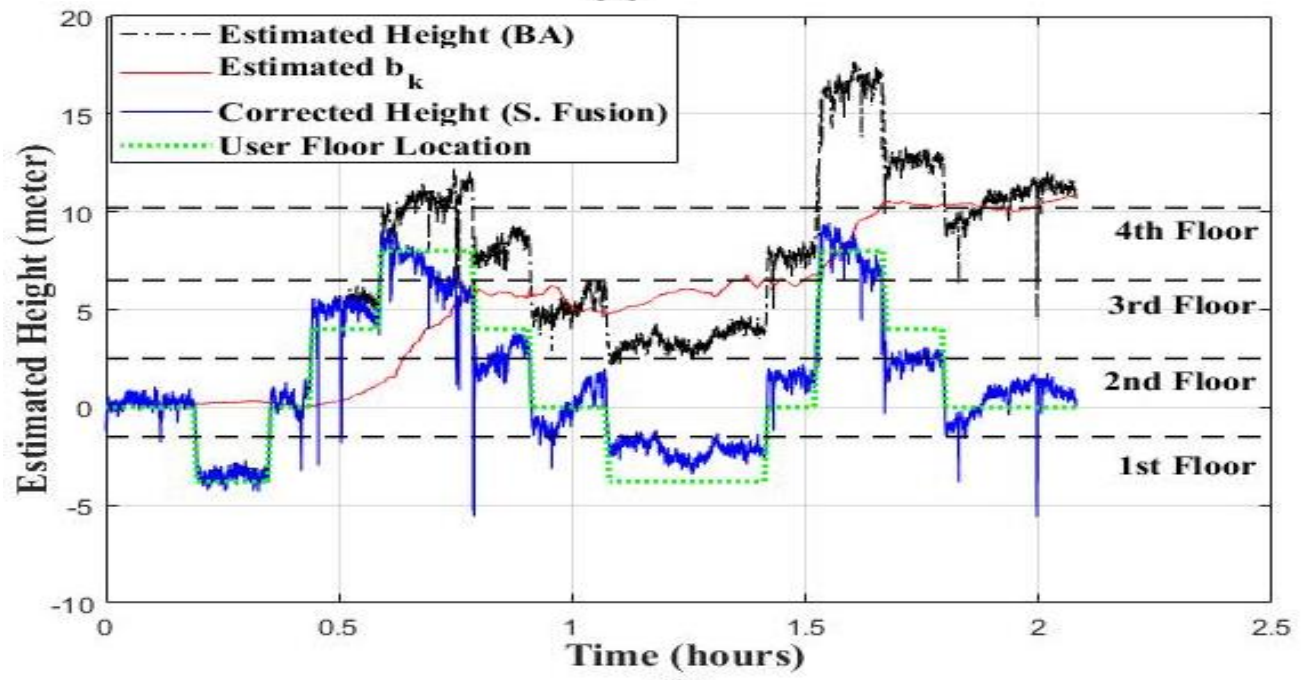

(c)

Figure 5.4: Estimated height for floor localization using sensor fusion algorithm in (a) ICT, (b) TFDL, and (c) MSC 


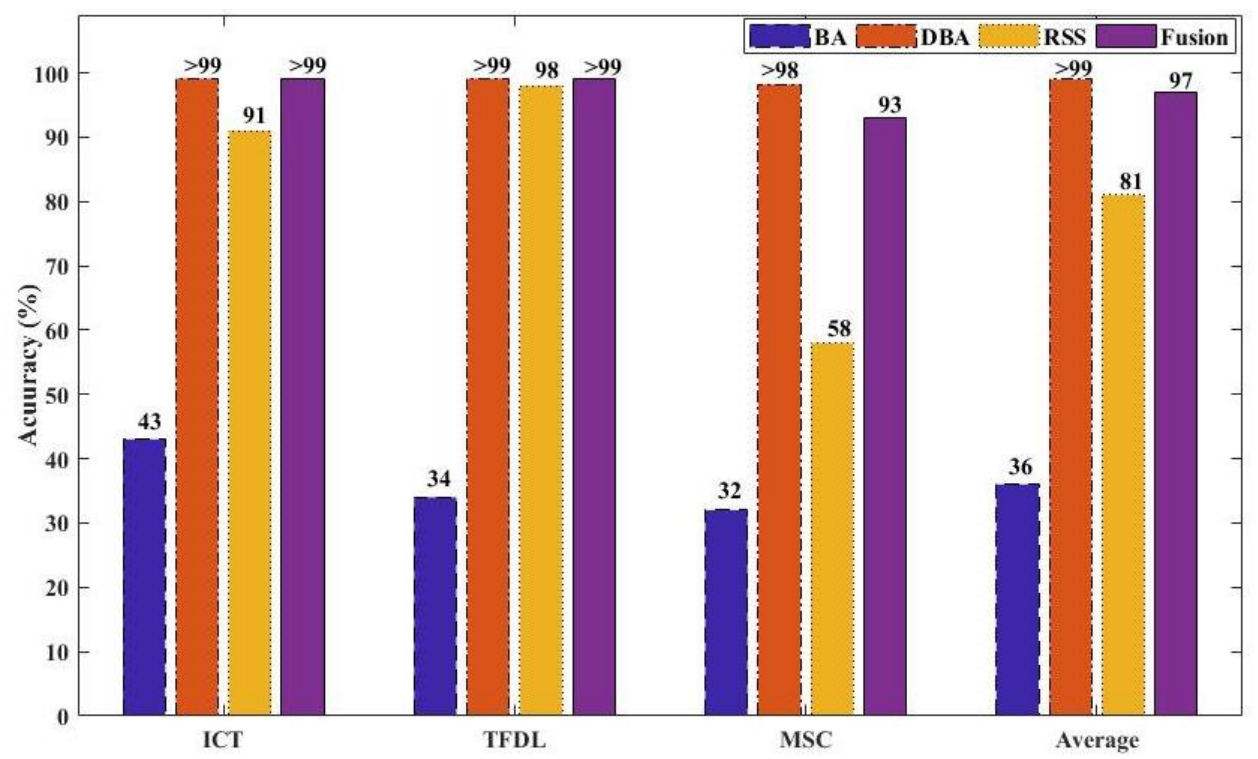

Figure 5.5: Comparison of floor localization accuracy

A comparison of accuracy of sensor fusion algorithm with those of BA-only, DBAonly and RSS-only floor localization methods is given in Figure 5.5 and Figure 5.6. As can be seen from Figure 5.5, the proposed sensor fusion algorithm overcomes the complimentary limitations of BA and RSS-based floor localization achieving over 97\% floor localization accuracy on average. Also, the performance of fusion algorithm is comparable to that of DBA-based system. Figure 5.6 shows the percentage of false floor localization over time for the performed measurements. As can be seen, BA introduces large errors for long-term measurements of several hours; whereas, DBA compensates for those errors. As mentioned in Chapter 4 and observed from Figure 5.6, RSS-based floor localization method introduces large errors over time inside a building with irregular structures and hollow interiors (i.e. MSC). From the figure, it is observed that the proposed sensor fusion algorithm has small errors in floor localization compared to the BA-only and RSS-only methods.

\subsection{Summary}

In this chapter, a theoretical framework for fusing BA and Wi-Fi RSS was developed for robust floor localization. The performance of the proposed sensor fusion algorithm was analyzed in comparison with those of BA-only, DBA-only, and RSS-only methods. The 

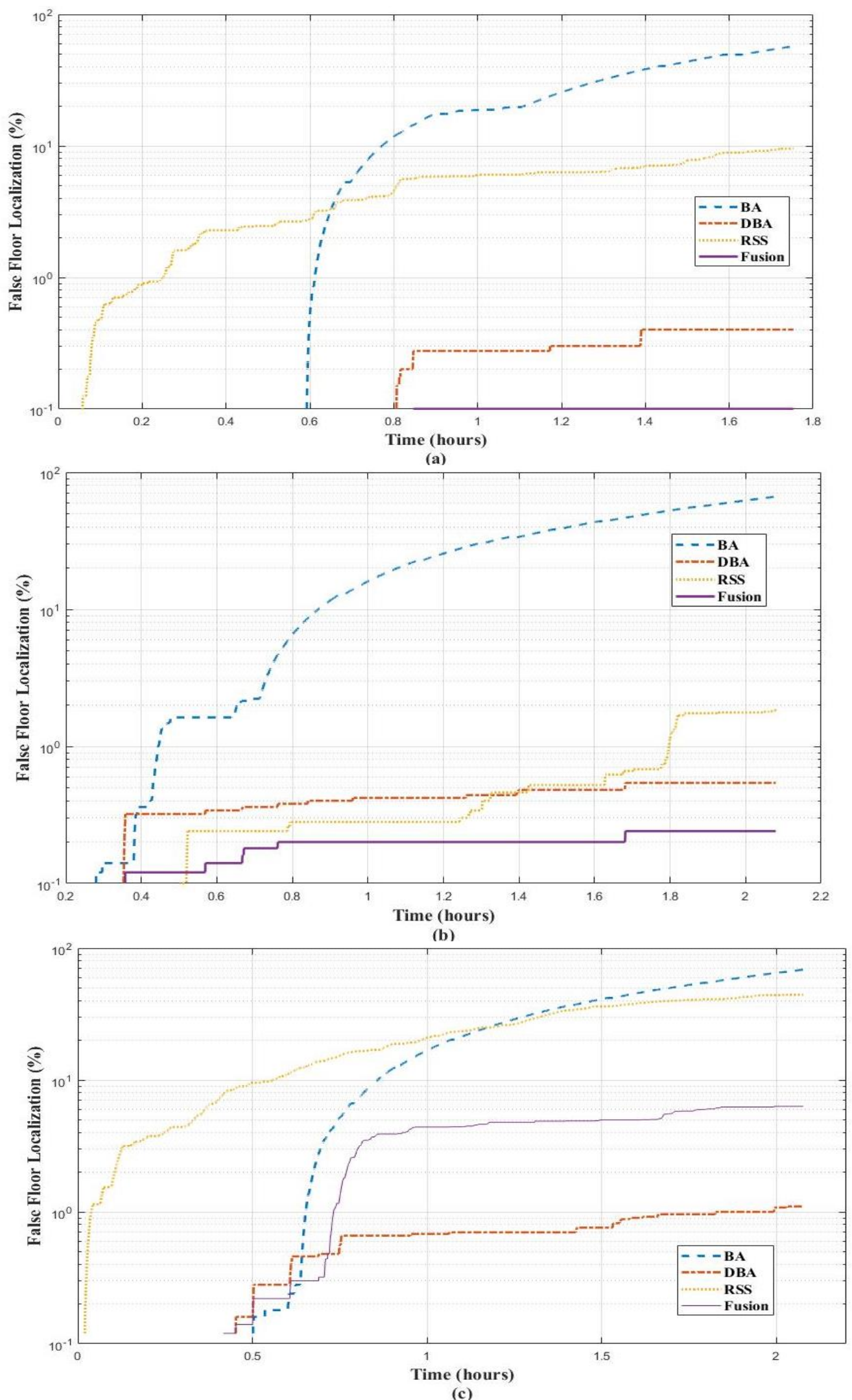

Figure 5.6: Percentage of false floor localization in (a) ICT, (b) TFDL, and (c) MSC 
chapter demonstrated that using the proposed sensor fusion algorithm the changes in bias in BA can be accurately estimated and tracked. Utilizing Wi-Fi RSS observables through a KF, the sensor fusion algorithm can compensate for the errors observed in BA. Unlike RSS-based floor localization methods, the proposed algorithm achieved 93\% or higher floor localization accuracy for all performed measurements irrespective of the building structure. The proposed algorithm is compatible with the state-of-the-art smartphones and can achieve accuracy comparable to that of DBA-based method without additional infrastructure. 


\section{Chapter 6: Conclusion}

\subsection{Thesis Summary}

A sensor fusion based framework for floor localization was developed based on using barometric pressure and Wi-Fi received signal strength (RSS) measurements. The proposed sensor fusion algorithm involved development of a new BA signal model, which was formulated based on extensive Allan deviation (ADEV) analysis of Micro-electromechanical-systems (MEMS) barometers. As well, a new Monte Carlo Bayesian Inference (MCBI) framework was developed for interpreting Wi-Fi RSS measurements. The performance of the proposed sensor fusion algorithm was then quantified and compared with barometric altimetry (BA)-only, differential barometric altimetry (DBA)-only, and RSS-only floor localization algorithms through measurements. The major conclusions of this dissertation are given in Section 6.2 and the recommendations for future work are outlined in Section 6.3.

\subsection{Conclusions}

The following major conclusions can be made based on the results obtained in the previous chapters:

- BA can be readily implemented for floor localization; nevertheless, it is highly influenced by approaching weather, air-conditioning, wind, temperature, etc. ADEV analysis performed in this dissertation revealed two categories of underlying random processes in $\mathrm{BA}$, which are: WGN, and Colored Noise of $1 / f^{\alpha} \mathrm{PSD}$, with exponent $\alpha \geq 1$.

- Since BA readings are prone to drift errors due to local changes in the air pressure, BA lacks the required accuracy to be used as a standalone technique in floor localization for long-term measurements of several hours.

- DBA achieves high accuracy (on average $>99 \%$ for the performed measurements in this dissertation) in floor localization by real-time differencing of measured air pressures from reference and mobile barometers. However, DBA requires installation of reference nodes and ad-hoc network for real-time information 
exchange. As such, DBA cannot be implemented readily and on a global scale for floor localization.

- Performance of RSS-based floor localization methods depends on the availability of APs, quality of offline calibration, and building structure. The performance deteriorates drastically inside a building with irregular structure and hollow interiors. For the performed measurements, RSS-based methods achieved $\leq 60 \%$ accuracy in MSC (as shown in Chapter 4), which as mentioned in Chapter 3 is a typical of a modern mall with an open multi-level design with large hollow spaces.

- Distribution of Wi-Fi RSS observables from plurality of APs inside a building resembles a log-normal distribution. Based on the distribution, an RSS based MCBI framework was formulated for floor localization in this dissertation that outperforms two existing ML-based methods.

- The proposed sensor fusion framework compensates for the drawbacks of BA-only and RSS-only floor localization methods. Also, the proposed method can be conveniently implemented with the existing infrastructures.

- The proposed sensor fusion algorithm outperforms both BA-only and RSS-only floor localization methods with an average accuracy of $97 \%$ for the performed measurements. The algorithm can be used for floor localization inside buildings with irregular structures and hollow interiors without introducing large errors.

\subsection{Recommendations for Future Work}

In future, more research can be conducted involving the proposed sensor fusion algorithm. Following is a list of recommendations for future work:

- The proposed sensor fusion algorithm can be integrated into a 3-dimensional (3D) indoor localization system to completely determine the position of a smartphone user and to improve the accuracy of the localization system.

- RSS-based maximum posteriori probability (MAP) classifier is an integrated part of the proposed sensor fusion algorithm. For floor localization, MAP classifier draws samples from areas with high probability (i.e. considers floors that are associated with the APs discovered during a recent wireless network scan) as shown 
in (4.12). This step can be further modified by considering user activities during a day. For instance, at lunch hours inside a mall it is more probable that a shopper will be located on a floor with food court. Exploring this aspect and incorporating this information may further improve the floor localization accuracy.

- Cooperative sharing of RSS observables along with range measurements between the neighboring mobile nodes can be incorporated in future measurements. Incorporating cooperative sharing is expected to further improve localization system.

- In this dissertation, for RSS measurements, a 2.4 GHz RF transceiver was used. In future, RSS observables from both Wi-Fi $2.4 \mathrm{GHz}$ and $5 \mathrm{GHz}$ bands can be considered during measurements to observe the impact on the performance of the proposed algorithm.

- This dissertation only considered a scenario of locating smartphone user floor location inside a building. The proposed sensor fusion algorithm can also be incorporated in robotics for indoor navigation.

- Crowdsourcing technique can be introduced to make the proposed floor localization algorithm more dynamic. Nevertheless, note that integrating crowdsourcing technique will increase the complexity of the localization system. 


\section{References}

[1] Y. Li, P. Zhang, X. Niu, Y. Zhuang, H. Lan and N. El-Sheimy, "Real-time Indoor Navigation using Smartphone Sensors," in 2015 International Conference on Indoor Positioning and Indoor Navigation (IPIN), Banff, Canada, 2015.

[2] F. Zhao, H. Luo, X. Zhao, Z. Pang and H. Park, "HYFI: Hybrid Floor Identification Based on Wireless Fingerprinting and Barometric Pressure," IEEE Transactions on Industrial Informatics, vol. 13, no. 1, pp. 330-341, 2017.

[3] V. Dehghanian and M. Lowe, "RSS-INS Integration for Cooperative Indoor Positioning," in International Conference on Indoor Positioning and Indoor Navigation (IPIN), Alcalá de Henares, Spain, 2016.

[4] R. Ichikari, L. C. M. Ruiz, M. Kourogi, T. Kurata, T. Kitagawa and S. Yoshii, "Indoor Floor-Level Detection by Collectively Decomposing Factors of Atmospheric Pressure," in 2015 International Conference on Indoor Positioning and Indoor Navigation (IPIN), Banff, Canada, 2015.

[5] V. Dehghanian, Generalized Diversity Gain of a Mobile Antenna, Calgary: Electrical and Computer Engineering, University of Calgary, 2011.

[6] N. Amani, V. Dehghanian and J. Nielsen, "User-induced antenna variation and its impact on the performance of RSS-based indoor positioning," in 2016 IEEE Canadian Conference on Electrical and Computer Engineering (CCECE), Vancouver, BC, Canada, 2016.

[7] V. Dehghanian, J. Nielsen and G. Lachapelle, "Combined spatial-polarization correlation function for indoor multipath environments," in 2010 7th International Symposium on Wireless Communication Systems (ISWCS), York, UK, 2010. 
[8] M. Zhang, L. Pei and X. Deng, "GraphSLAM-based Crowdsourcing framework for indoor Wi-Fi fingerprinting," in 2016 Fourth International Conference on Ubiquitous Positioning, Indoor Navigation and Location Based Services (UPINLBS), Shanghai, China, 2016.

[9] A. Rai, K. K. Chintalapudi, V. N. Padmanabhan and R. Sen, "Zee: zero-effort crowdsourcing for indoor localization," in Mobicom '12 Proceedings of the 18th annual international conference on Mobile computing and networking, Istanbul, Turkey, 2012.

[10] D. E. Bolanakis, "Evaluating performance of MEMS barometric sensors in differential altimetry systems," IEEE Aerospace and Electronic Systems Magazine, vol. 32, no. 9, pp. 34 - 39, 2017.

[11] N. Hayashi, Augmentation of GPS with a barometer and a heading rate gyroscope for urban vehicular navigation [microform], Calgary: University of Calgary, 1996.

[12] J. Rantakokko, J. Rydell, P. Strömbäck, P. Händel, J. Callmer, D. Törnqvist, F. Gustafsson, M. Jobs and M. Grudén, "Accurate and reliable soldier and first responder indoor positioning: multisensor systems and cooperative localization," IEEE Wireless Communications, vol. 18, no. 2, pp. 10-18, 2011.

[13] D. A. Grejner-Brzezinska, C. K. Toth, T. Moore, J. F. Raquet, M. M. Miller and A. Kealy, "Multisensor Navigation Systems: A Remedy for GNSS Vulnerabilities?," Proceedings of the IEEE, vol. 104, no. 6, pp. 1339 - 1353, 2016.

[14] K. Liu, Y. Wang and J. Wang, "Differential Barometric Altimetry Assists Floor Identification in WLAN Location Fingerprinting Study," in Principle and Application Progress in Location-Based Services, Springer, 2014, pp. 21-29.

[15] C. Bollmeyer, T. Esemann, H. Gehring and H. Hellbruck, "Precise Indoor Altitude Estimation based on differential barometric Sensing for wireless Medical Applications," in 2013 IEEE International Conference on Body Sensor Networks (BSN), Cambridge, MA, USA, 2013. 
[16] M. Won, A. Mishra and S. H. Son, "HybridBaro: Mining Driving Routes Using Barometer Sensor of Smartphone," IEEE Sensors Journal, vol. 17, no. 19, pp. 6397 - 6408, 2017.

[17] S. Zihajehzadeh, T. J. Lee, J. K. Lee, R. Hoskinson and E. J. Park, "Integration of MEMS Inertial and Pressure Sensors for Vertical Trajectory Determination," IEEE Transactions on Instrumentation and Measurement, vol. 64, no. 3, pp. 804-814, 2015 .

[18] B. Shin, C. Kim, J. Kim, S. Lee, C. Kee, H. S. Kim and T. Lee, "Motion RecognitionBased 3D Pedestrian Navigation System Using Smartphone," IEEE Sensors Journal, vol. 16, no. 18, pp. 6977 - 6989, 2016.

[19] S. Wei, G. Dan and H. Chen, "Altitude data fusion utilising differential measurement and complementary filter," IET Science, Measurement \& Technology, vol. 10, no. 8, pp. 874-879, 2016.

[20] F. Alsehly, T. Arslan and Z. Sevak, "Indoor Positioning with floor determination in Multi Story Buildings," in International Conference on Indoor Positioning and Indoor Navigation (IPIN) 2011, Guimaraes, Portugal, 2011.

[21] X. Shen, Y. Chen, J. Zhang, L. Wang, G. Dai and T. He, "BarFi: Barometer-Aided Wi-Fi Floor Localization Using Crowdsourcing," in 2015 IEEE 12th International Conference on Mobile Ad Hoc and Sensor Systems, Dallas, TX, 2015.

[22] F. Haque, V. Dehghanian and A. O. Fapojuwo, "Sensor Fusion for Floor Detection," in The 8th IEEE Annual Information Technology, Electronics and Mobile Communication Conference, Vancouver, 2017.

[23] P. Gupta, S. Bharadwaj, S. Ramakrishnan and J. Balakrishnan, "Robust Floor Determination for Indoor Positioning," in 2014 Twentieth National Conference on Communications (NCC), Kanpur, 2014. 
[24] Y. Tian, B. Denby, I. Ahriz, P. Roussel and G. Dreyfus, "Hybrid Indoor Localization using GSM Fingerprints, Embedded Sensors and a Particle Filter," in 11th International Symposium on Wireless Communications Systems (ISWCS), Barcelona, Spain, 2014.

[25] A. Razavi, M. Valkama and E. S. Lohan, "Robust Statistical Approaches for RSSBased Floor Detection in Indoor Localization," Sensors, vol. 16, no. 6, 2016.

[26] M. A. A. Rahman, M. Dashti and J. Zhang, "Floor Determination for Positioning in Multi-story Building," in IEEE Wireless Communications and Networking Conference (WCNC), 2014, Istanbul, Turkey, 2014.

[27] F.-M. Jhuang, C.-F. Hung, C.-C. Tuan, Y.-C. Wu and F.-Y. Leu, "An AP Selection with RSS Standard Deviation for Indoor Positioning in Wi-Fi," in 9th International Conference on Innovative Mobile and Internet Services in Ubiquitous Computing, Blumenau, Brazil, 2015.

[28] T. Vongsuteera, K. N. Nakorn and K. Rojviboonchai, "Floor Localization Algorithm utilizing Different Order of Access Point from Wi-Fi Signal Fingerprint," in 2016 13th International Conference on Electrical Engineering/Electronics, Computer, Telecommunications and Information Technology (ECTI-CON), Chiang Mai, Thailand, 2016.

[29] K. Maneerat and C. Prommak, "Floor Estimation Algorithm for Wireless Indoor Multi-Story Positioning Systems," in 11th International Conference on Electrical Engineering/Electronics, Computer, Telecommunications and Information Technology (ECTI-CON), Nakhon Ratchasima, Thailand, 2014.

[30] K. Maneerat and C. Prommak, "An Enhanced Floor Estimation Algorithm for Indoor Wireless Localization Systems Using Confidence Interval Approach," International Journal of Computer, Electrical, Automation, Control and Information Engineering, vol. 8, no. 7, p. 1182-1186, 2014. 
[31] K. Maneerat and C. Prommak, "Floor Determination Algorithm with Node Failure Consideration for Indoor Positioning Systems," in Proceedings of the 8th International Conference on signal processing systems, Auckland, New Zealand, 2016.

[32] K. Maneerat, K. Kaemarungsi and C. Prommak, "Robust Floor Determination Algorithm for Indoor Wireless Localization Systems under Reference Node Failure," Mobile Information Systems, vol. 2016, 2016.

[33] J. Yoo, K. H. Johansson and H. J. Kim, "Indoor Localization Without a Prior Map by Trajectory Learning From Crowdsourced Measurements," IEEE TRANSACTIONS ON INSTRUMENTATION AND MEASUREMENT, vol. PP, no. 99, pp. 1-11, 2017.

[34] B.-A. Chen, P.-H. Tseng, K.-T. Feng and T.-S. Wang, "Fingerprinting-based Dynamic RSS Adjustment for Time-variant Indoor Positioning Systems," in 2016 IEEE International Conference on Consumer Electronics-Taiwan (ICCE-TW), Nantou, Taiwan, 2016.

[35] Y. Li, J. Chen, Y. Shi, Y. Cheng and L. Wang, "WiFi-Assisted Multi-Floor Indoor Localization with Inertial Sensors," in 2016 8th International Conference on Wireless Communications \& Signal Processing (WCSP), Yangzhou, China, 2016.

[36] P. Tang, Z. Huang and J. Lei, "Fingerprint localization using WLAN RSS and magnetic field with landmark detection," in 3rd International Conference on Computational Intelligence \& Communication Technology (CICT), Ghaziabad, India, 2017.

[37] N. Etemadyrad and J. K. Nelson, "A Sequential Detection Approach to Indoor Positioning Using RSS-Based Fingerprinting," in IEEE Global Conference on Signal and Information Processing (GlobalSIP), Washington, DC, USA, 2016. 
[38] S. He and S.-H. G. Chan, "Wi-Fi Fingerprint-Based Indoor Positioning: Recent Advances and Comparisons," IEEE Communications Surveys \& Tutorials, vol. 18, no. 1, pp. 466 - 490, 2016.

[39] G. Retscher, "Augmentation of Indoor Positioning Systems with a Barometric Pressure Sensor for Direct Altitude Determination in a Multi-storey Building," Cartography and Geographic Information Science, vol. 34, no. 4, pp. 305-310, 2007.

[40] K. Muralidharan, A. J. Khan, A. Misra, R. K. Balan, S. Agarwal, R. K. Balan and S. Agarwal, "Barometric Phone Sensors - More Hype Than Hope!," in HotMobile '14 Proceedings of the 15th Workshop on Mobile Computing Systems and Applications, Santa Barbara, California, 2014.

[41] H. Xia, X. Wang, Y. Qiao, J. Jian and Y. Chang, "Using Multiple Barometers to Detect the Floor Location of Smart Phones with Built-in Barometric Sensors for Indoor Positioning," Sensors, vol. 15, no. 4, pp. 7857-7877, April 2015.

[42] H. Ye, T. Gu, X. Tao and J. Lu, "B-Loc: Scalable Floor Localization using Barometer on Smartphone," in IEEE 11th International Conference on Mobile Ad Hoc and Sensor Systems, Philadelphia, PA, USA, 2014.

[43] H. Ye, T. Gu, X. Tao and J. Lu, "Scalable floor localization using barometer on smartphone," Wireless Communications and Mobile Computing, vol. 16, no. 16, pp. 2557-2571, 2016.

[44] L. Bedogni, F. Franzoso and L. Bononi, "A Self-Adapting Algorithm Based on Atmospheric Pressure to Localize Indoor Devices," in IEEE Global Communications Conference (GLOBECOM), Washington, DC, USA, 2016.

[45] D. E. Bolanakis, K. T. Kotsis and T. Laopoulos, "A Prototype Wireless Sensor Network System for a Comparative Evaluation of Differential and Absolute Barometric Altimetry," IEEE Aerospace and Electronic Systems Magazine, vol. 30, no. 11 , pp. $20-28,2015$. 
[46] H. Wang, H. Lenz, A. Szabo, U. D. Hanebeck and J. Bamberger, "Fusion of Barometric Sensors, WLAN Signals and Building Information for 3-D Indoor/Campus Localization," in IEEE International Conference on Multisensor Fusion and Integration for Intelligent Systems (MFI 2006), Heidelberg, Germany, 2006.

[47] C. Luo, H. Hong, M. C. Chan, J. Li, X. Zhang and Z. Ming, "MPiLoc: Selfcalibrating Multi-floor Indoor Localization Exploiting Participatory Sensing," IEEE Transactions on Mobile Computing, vol. PP, no. 99, 2017.

[48] H. J. Ai, M. Y. Liu, Y. M. Shi and J. Q. Zhao, "Floor Identification with Commercial Smartphones In Wifi-Based Indoor Localization System," The International Archives of the Photogrammetry, Remote Sensing and Spatial Information Sciences, Vols. XLI-B4, pp. 573-577, 2016.

[49] X. Bao, Z. Xiong, S. Sheng, Y. Dai, S. Bao and J. Liu, "Barometer measurement error modeling and correction for UAH altitude tracking," in 2017 29th Chinese Control And Decision Conference (CCDC), Chongqing, China, 2017.

[50] IEEE, "IEEE Recommended Practice for Inertial Sensor Test Equipment, Instrumentation, Data Acquisition, and Analysis," IEEE Std. 1554-2005, pp. 1-145, 2013.

[51] IEEE, "IEEE Standard Definitions of Physical Quantities for Fundamental Frequency and Time Metrology---Random Instabilities," IEEE Std. 1139-2008 (Revision of IEEE Std 1139-1999), pp. 1-50, 2009.

[52] IEEE, "IEEE Standard Specification Format Guide and Test Procedure for SingleAxis Interferometric Fiber Optic Gyros -- Corrigendum 1: Figure 1 and Subclauses 5.3.4, 8.3, 12.11.4.3.2, 12.11.4.3.3, 12.11.4.3.4, 12.12.3.1, and 12.12.4.1," IEEE Std. 952-1997/Cor 1-2016 (Corrigendum to IEEE Std. 952-1997), pp. 1-14, 2017. 
[53] N. El-Sheimy, H. Hou and X. Niu, "Analysis and Modeling of Inertial Sensors Using Allan Variance," IEEE Transactions on Instrumentation and Measurement, vol. 57, no. 1, pp. 140-149, 2008.

[54] Bosch Sensortec, "Datasheet BMP180 Digital Pressure Sensor," Bosch Sensortec, Reutlingen, Germany, 2013.

[55] Bosch Sensortec, "Datasheet BMP280 Digital Pressure Sensor," Bosch Sensortec, Reutlingen, Germany, 2015.

[56] Bosch Sensortec, "BME280 Combined humidity and pressure sensor," Bosch Sensortec, Reutlingen, Germany, 2016.

[57] Freescale Semiconductor, Inc., "Datasheet: Xtrinsic MPL3115A2 I2C Precision Altimeter," Freescale Semiconductor, Inc. , Austin, Texas, United States, 2013.

[58] Freescale Semiconductor Inc., "Allan Variance: Noise Analysis for Gyroscopes," NXP, Austin, Texas, United States, 2015.

[59] M. S. Keshner, "1/f noise," Proceedings of the IEEE, vol. 70, no. 3, pp. 212 - 218, 1982.

[60] J. R. M. Hosking, "Fractional differencing," Biometrika, vol. 68, no. 1, pp. 165-176, 1981.

[61] S. M. Kay, "Statistical Decision Theory I," in Fundamentals of Statistical Signal Processing: Detection Theory, Upper Saddle River, New Jersey, Prentice Hall PTR, 1998, pp. 60-92.

[62] Cypress Semiconductor Corporation, "CYW43362 Single-Chip IEEE 802.11 TM b/g/n MAC/Baseband/Radio," Cypress Semiconductor Corporation, San Jose, California, United States, 2017. 
[63] J. H. Lee and R. M. Buehrer, "Fundamentals of Received Signal Strength - Based Position Location," in Handbook of Position Location: Theory, Practice and Advances, Hoboken, New Jersey, John Wiley \& Sons, Inc., 2012, pp. 359-394.

[64] D. P. Smith, "Fundamentals of the Wireless Channel," in Wireless Propagation in the Near-Ground Forest Environment, Calgary, University of Calgary, 2015, pp. 1240.

[65] K. P. Murphy, Machine learning: a probabilistic perspective, Cambridge, MA: MIT Press, 2012.

[66] C. M. Bishop, Pattern Recognition and Machine Learning (Information Science and Statistics), New York: Springer, 2006.

[67] J. L. Puga, M. Krzywinski and N. Altman, "Points of Significance: Bayes' theorem," Nature Methods, vol. 12, pp. 277-278, 2015.

[68] J. A. d. Peral-Rosado, M. Bavaro, J. A. Lopez-Salcedo, G. Seco-Granados, P. Chawdhry, J. Fortuny-Guasch, P. Crosta, F. Zanier and M. Crisci, "Floor Detection with Indoor Vertical Positioning in LTE Femtocell Networks," in 2015 IEEE Globecom Workshops (GC Wkshps), San Diego, CA, USA, 2015.

[69] M. S. Grewal and A. P. Andrews, "General Information," in Kalman Filtering Theory and Practice Using Matlab, Hoboken, New Jersey, John Wiley \& Sons, Inc., 2008, pp. 1-25.

[70] S. M. Kay, Fundamentals of Statistical Signal Processing: Estimation Theory, Upper Saddle River, New Jersey: Prentice Hall PTR, 1993.

[71] L. Zhang and X. Peng, "Time series estimation of gas sensor baseline drift using ARMA and Kalman based models," Sensor Review, vol. 36, no. 1, pp. 34-39, 2016. 
[72] Z. Diao, H. Quan, L. Lan and Y. Han, "Analysis and compensation of MEMS gyroscope drift," in Sensing Technology (ICST), 2013 Seventh International Conference on, Wellington, New Zealand, 2013.

[73] K. Wang, S. Xiong and Y. Li, "Modeling with noises for inertial sensors," in 2012 IEEE/ION Position, Location and Navigation Symposium, Myrtle Beach, SC, USA, 2012.

[74] E. Rossi, "Stationary ARMA Processes, University of Pavia," 2007. [Online]. Available:

http://economia.unipv.it/pagp/pagine_personali/erossi/macroeconometria_stationar y_arma.pdf. [Accessed 29 May 2018].

[75] A. Nosedal, "The Moving Average Models MA(1) and MA(2), University of Toronto," $10 \quad$ February $2016 . \quad$ [Online]. Available: https://mcs.utm.utoronto.ca/ nosedal/sta457/ma1-and-ma2.pdf. [Accessed 15 November 2017]. 


\section{APPENDIX A}

\section{ARMA Model Formulation for MEMS Barometer Measurements}

In the signal processing community, it is a well-known practice to use a linear differential model, such as Autoregressive Moving Average (ARMA) for signal modeling. Also, it is a common practice to use ARMA to model various sensors, e.g. gyroscope, accelerometer, gas sensor etc., measurements [71, 72, 73]. ARMA has a structure of including both uncorrelated (WGN) and correlated (low-frequency noise) error terms. In this section, barometer measurement will be modeled using a first order ARMA or $\mathrm{AR}(1) \mathrm{MA}(1)$ because it is more tractable and less complex than any of its higher order model. Note that, caution is required when applying ARMA model to represent the nonstationary processes such as barometer measurement. An AR(1)MA(1) model to represent barometer measurement can be defined as

$$
\tilde{h}[n]=\phi \tilde{h}[n-1]+\theta u[n-1]+u[n] ; n=0,1,2, \ldots M
$$

where $\tilde{h}$ was defined as measured height in Chapter $3, \phi$ and $\theta$ are two coefficients, and $u[n]$ is a zero mean WGN process with variance $\sigma_{u}{ }^{2}$.

The value of $\phi$ in (A.1) can be estimated using the Yule-Walker equation. Subsequently, $\phi$ is utilized to determine the value of $\theta$ and $\sigma_{u}{ }^{2}$. According to [70], the Yule-Walker equation to determine $\phi$ is represented as

$$
\widehat{\phi} \hat{r}_{\widetilde{h} \tilde{h}}[k-1]=\hat{r}_{\widetilde{h} \tilde{h}}[k] ; k=2, \ldots, M
$$

where $\hat{\phi}$ denotes the estimated value of $\phi, \hat{r}_{\widetilde{h} \tilde{h}}$ is the estimated autocorrelation of $\tilde{h}[n]$. Note that, as (A.1) incorporates an MA(1) parameter, (A.2) is not valid for $k=1$. The autocorrelations of $\tilde{h}[n]$ are given as [74]

$$
r_{\widetilde{h} \tilde{h}}[0]=E(\tilde{h}[n] \tilde{h}[n])=\frac{\sigma_{u}^{2}\left(2 \phi \theta+\theta^{2}+1\right)}{1-\phi^{2}}
$$




$$
\begin{gathered}
r_{\widetilde{h} \tilde{h}}[1]=E(\tilde{h}[n] \tilde{h}[n-1])=\phi r_{\widetilde{h} \tilde{h}}[0]+\theta \sigma_{u}{ }^{2} \\
r_{\widetilde{h} \tilde{h}}[k]=E(\tilde{h}[n] \tilde{h}[n-k])=\phi^{k-1} r_{\widetilde{h} \tilde{h}}[1] ; \text { for } k>1
\end{gathered}
$$

From (A.2), $\widehat{\phi}$ can be determined as

$$
\widehat{\phi}=\frac{\hat{r}_{\widetilde{h} \tilde{h}}[k]}{\hat{r}_{\widetilde{h} \tilde{h}}[k-1]} ; k=2, \ldots, M
$$

Next, to derive an equation to estimate $\theta$, first an MA(1) process is considered and it is defined as

$$
y[n]=c_{1} \varpi[n-1]+\varpi[n]
$$

where $\varpi[n]$ is a zero mean WGN process with variance ${\sigma_{\varpi}}^{2}$ and $c_{1}$ is a coefficient. The autocorrelation (denoted with $r_{y y}$ ) for the process in (A.5) is given as [75]

$$
\begin{gathered}
E(y[n] y[n])=r_{y y}[0]=\left(1+c_{1}{ }^{2}\right){\sigma_{\varpi}}^{2} \\
E(y[n] y[n-1])=r_{y y}[1]=c_{1} \sigma_{\varpi}{ }^{2} \\
E(y[n] y[n-k])=r_{y y}[k]=0 ; \text { for } k>1
\end{gathered}
$$

Using $r_{y y}[0]$ and $r_{y y}[1]$ from (A.6) it can be shown that

$$
c_{1}=\frac{r_{y y}[0] \pm \sqrt{\left(r_{y y}[0]\right)^{2}-4\left(r_{y y}[1]\right)^{2}}}{2 r_{y y}[1]}
$$

By comparing the autocorrelation of $y[n]=z[n]-\phi z[n-1]=\theta w[n-1]+u[n]$ with the autocorrelation properties stated in (A.6), it can be shown that $y[n]$ is a MA(1) process; and as a result, $\theta$ can be estimated according to

(A.7), i.e.

$$
\hat{\theta}=\frac{r_{\dot{y} \dot{y}}[0] \pm \sqrt{\left(r_{\dot{y} y}[0]\right)^{2}-4\left(r_{\dot{y} y}[1]\right)^{2}}}{2 r_{\dot{y} y}[1]}
$$


Later, the value of $\hat{\phi}$ and $\hat{\theta}$ can be replaced in (A.3) to estimate $\sigma_{u}{ }^{2}$. The software MATLAB has a function called ARIMA, which can be used to estimate $\phi, \theta$, and $\sigma_{u}{ }^{2}$ from a given dataset.

A more general way to represent the ARMA process is its state space (SS) representation. The SS representations for (A.1) can be given as

$$
\begin{gathered}
\tilde{h}[n]=h+b[n]+w[n] ; n=1,2, \ldots M \\
b[n]=a b[n-1]+v[n]
\end{gathered}
$$

where $w[n]$ and $v[n]$ (known as excitation or driving noise) are independent zero mean WGN processes with variances ${\sigma_{w}}^{2}$ and ${\sigma_{b}}^{2}$; i.e. $w[n] \sim \mathcal{N}\left(0,{\sigma_{w}}^{2}\right)$ and $v[n] \sim \mathcal{N}\left(0,{\sigma_{b}}^{2}\right)$. Moreover, (A.10) represents a first order Autoregressive (AR) model, also known as firstorder hidden Markov model, where the condition $|a|<1$ must be satisfied for the stability of the model. Otherwise, mean or/and variance of $b[n]$ grows with $n$; hence, the process becomes non-stationary [70].

The relations between parameters $\phi, \theta, \sigma_{u}{ }^{2}$ and $a, \sigma_{w}{ }^{2}$, and $\sigma_{b}{ }^{2}$ can be derived by following a few mathematical steps. From (A.4) it can be written

$$
\frac{r_{\widetilde{h} \tilde{h}}[3]}{r_{\widetilde{h} \tilde{h}}[2]}=\phi
$$

Again, from (A.9) and (A.10),

$$
\begin{aligned}
& r_{\tilde{h} \tilde{h}}[3]=E\{\tilde{h}[n] \tilde{h}[n-3]\} \\
& \quad=E\{(a b[n-1]+u[n]+v[n])(b[n-3]+v[n-3])\} \\
& \text { or, } r_{\widetilde{h} \tilde{h}}[3]=a r_{b b}[2]
\end{aligned}
$$

where $r_{b b}$ is the autocorrelation function of $b[n]$. Similarly,

$$
\begin{aligned}
& r_{\tilde{h} \tilde{h}}[2]=E\{\tilde{h}[n] \tilde{h}[n-2]\} \\
&= E\{(a b[n-1]+u[n]+v[n])(b[n-2]+v[n-2])\} \\
& \text { or, } r_{\widetilde{h} \tilde{h}}[2]=a r_{b b}[1]
\end{aligned}
$$


Moreover, using the autocorrelation properties of $\mathrm{AR}(1)$ process and from (A.12) and (A.13), the following is obtained.

$$
\frac{r_{\widetilde{h} \tilde{h}}[3]}{r_{\widetilde{h} \tilde{h}}[2]}=\frac{r_{b b}[2]}{r_{b b}[1]}=a
$$

From the comparison of (A.11) and (A.14), we have

$$
a=\phi
$$

From (A.6), for the MA(1) process $y^{\prime}[n]=\tilde{h}[n]-\phi \tilde{h}[n-1]=\theta u[n-1]+u[n]$, the following can be written

$$
\begin{gathered}
r_{\dot{y} y}[0]=\left(1+\theta^{2}\right) \sigma_{u}{ }^{2} \\
r_{y y ́}[1]=\theta \sigma_{u}{ }^{2}
\end{gathered}
$$

Also, from (A.9), (A.10), and (A.15), the following can be written

$$
\begin{aligned}
y^{\prime}[n] & =\tilde{h}[n]-\phi \tilde{h}[n-1] \\
= & \tilde{h}[n]-a \tilde{h}[n-1] \\
= & v[n]+w[n]-a w[n-1]
\end{aligned}
$$

(A.18) represents an $\mathrm{MA}(1)$ process and its first two autocorrelation functions can be shown as,

$$
\begin{gathered}
r_{\dot{y} y}[0]=\sigma_{b}{ }^{2}+\left(1+a^{2}\right) \sigma_{w}{ }^{2} \\
r_{y ́ y}[1]=-a \sigma_{w}{ }^{2}
\end{gathered}
$$

Comparing (A.16) with (A.19), and (A.17) with (A.20), the following two expressions are derived, 


$$
\begin{gathered}
\sigma_{w}{ }^{2}=-\frac{\theta}{\phi} \sigma_{u}{ }^{2} \\
\sigma_{b}{ }^{2}=\left(1+\theta^{2}\right) \sigma_{u}{ }^{2}-\left(1+a^{2}\right) \sigma_{w}{ }^{2}
\end{gathered}
$$

Using (A.15), (A.21), and (A.22), the values of the parameters $a, \sigma_{w}$, and $\sigma_{b}$ were estimated from the same measurement sets that were collected using BMP180, BMP280, BME280, and MPL3115A2 for the ADEV curves. As mentioned before, caution is required when applying stationary ARMA model to a non-stationary process. The measurement sets were grouped into many small groups (each containing measurement of 30 seconds), for which the barometer measurement can be considered stationary. AR(1)MA(1) model was fitted to those small groups of measurements and an average was taken to estimate the values of $a, \sigma_{w}$, and $\sigma_{b}$. TABLE A.1 shows the estimated values of the parameters.

TABLE A.1

ESTIMATED AR(1)MA(1) PARAMETERS

\begin{tabular}{|c|c|c|c|}
\hline BP Sensor Model & $a$ & $\sigma_{w}$ & $\sigma_{b}$ \\
\hline BMP180 & 0.5705 & 0.3546 & 0.1771 \\
\hline BMP280 & 0.5622 & 0.1548 & 0.0980 \\
\hline BME280 & 0.5707 & 0.3186 & 0.1582 \\
\hline MPL3115A2 & 0.5983 & 0.1514 & 0.0717 \\
\hline
\end{tabular}

From TABLE A.1 it can be observed that the determined value of $\sigma_{w}$ and $\sigma_{b}$ are similar to what were found in Section 3.2 (see TABLE 3.1). Also, for all collected measurement set from the sensors under test, $|a|<1$. The condition $|a|<1$ did not hold true when AR(1)MA(1) model was fitted to the entire measurement set without dividing into small groups. 


\section{APPENDIX B}

\section{Measured Mean and Standard Deviation}

This appendix gives the measured RSS means $E\left[D_{\mathcal{F}}\right]$ and standard deviations $s t d\left[D_{\mathcal{F}}\right]$ as a function of mobile-AP floor separation $\mathcal{F}$ for all the three-buildings where measurements were collected. TABLE B.1, TABLE B.2, and TABLE B.3 show $E\left[D_{\mathcal{F}}\right]$ and $s t d\left[D_{\mathcal{F}}\right]$ for Information and Communications Technology (ICT) building, Taylor Family Digital Library (TFDL), and MacEwan Student Centre (MSC), respectively.

TABLE B. 1

$E\left[D_{\mathcal{F}}\right]$ AND $s t d\left[D_{\mathcal{F}}\right]$ FOR ICT BUILDING

\begin{tabular}{|c|c|c|c|c|}
\hline & $\mathcal{F}$ & Floor 1 & Floor 2 & Floor 3 \\
\hline \multirow{3}{*}{$E\left[D_{\mathcal{F}}\right](\mathrm{dBf})$} & 0 & 46 & 45 & 46 \\
\cline { 2 - 5 } & 1 & 40 & 41 & 44 \\
\cline { 2 - 5 } & $>1$ & 38 & - & 37 \\
\hline \multirow{3}{*}{$s t d\left[D_{\mathcal{F}}\right](\mathrm{dBf})$} & 0 & 6 & 6 & 6 \\
\cline { 2 - 5 } & 1 & 3 & 3 & 3 \\
\cline { 2 - 5 } & $>1$ & 1 & - & 5 \\
\hline
\end{tabular}

TABLE B.2

$E\left[D_{\mathcal{F}}\right]$ AND $s t d\left[D_{\mathcal{F}}\right]$ FOR TFDL

\begin{tabular}{|c|c|c|c|c|c|c|c|}
\hline & $\mathcal{F}$ & Floor 1 & Floor 2 & Floor 3 & Floor 4 & Floor 5 & Floor 6 \\
\hline \multirow{3}{*}{$E\left[D_{\mathcal{F}}\right](\mathrm{dBf})$} & 0 & 46 & 46 & 47 & 47 & 44 & 47 \\
\cline { 2 - 9 } & 1 & 42 & 41 & 40 & 39 & 39 & 39 \\
\cline { 2 - 9 } & $>1$ & - & - & - & - & - & - \\
\hline \multirow{3}{*}{$\operatorname{std}\left[D_{\mathcal{F}}\right](\mathrm{dBf})$} & 0 & 6 & 6 & 8 & 8 & 7 & 8 \\
\cline { 2 - 9 } & 1 & 4 & 4 & 2 & 3 & 2 & 2 \\
\cline { 2 - 9 } & $>1$ & - & - & - & - & - & - \\
\hline
\end{tabular}


TABLE B. 3

$E\left[D_{\mathcal{F}}\right]$ AND $s t d\left[D_{\mathcal{F}}\right]$ FOR MSC

\begin{tabular}{|c|c|c|c|c|c|}
\hline & $\mathcal{F}$ & Floor 1 & Floor 2 & Floor 3 & Floor 4 \\
\hline \multirow{3}{*}{$E\left[D_{\mathcal{F}}\right](\mathrm{dBf})$} & 0 & 48 & 47 & 45 & 45 \\
\cline { 2 - 7 } & 1 & 43 & 43 & 42 & 41 \\
\cline { 2 - 7 } & $>1$ & 38 & 39 & 40 & 41 \\
\hline \multirow{3}{*}{$\operatorname{std}\left[D_{\mathcal{F}}\right](\mathrm{dBf})$} & 0 & 9 & 7 & 6 & 7 \\
\cline { 2 - 7 } & 1 & 5 & 5 & 5 & 4 \\
\cline { 2 - 7 } & $>1$ & 3 & 3 & 2 & 3 \\
\hline
\end{tabular}




\section{APPENDIX C}

\section{Obtaining Access Points Information}

\section{through Site Surveys}

Before the RSS-based MAP classifier (introduced in Section 4.4) can be implemented it is required to know the addresses (i.e. MAC IDs) and floor locations of the APs. MAP classifier needs less storage space than that of fingerprinting and RSS-based multilateration methods to store APs information. For research and learning purpose, initially site surveys were conducted to identify the APs on each floor and this appendix describes that process. To identify the installed APs on each floor, RSS readings were measured in close proximity to the visible APs. In the scenarios where no APs were visible for a significant portion of a floor due to various obstacles, the floor was divided into multiple zones with a varying radius of 1-5 meters and RSS readings were measured to find any nearby AP in that zone. The sizes of the zones were dependent on the total area of the floor for which no APs were visible and the possibility of finding an AP in that area. For instance, inside TFDL, there are some outward corridors near the exit doors where it is less probable to have any AP; and so, zones with large radius were considered during measurements. Whereas, on the corridors of ICT building, where it is more probable to have APs installed, but could not be spotted during the measurements due to various obstacles, zones with small radius were used.

At each location, measurements lasted for several minutes, and in post-processing stage, an average was taken over the entire period to mitigate the effect of multipath signals. A measurement performed in proximity to an AP located on the second floor of MSC is shown in Figure C.1. The figure shows MAC IDs of the visible APs at that location and their corresponding average RSS. Due to security reasons, the MAC IDs are shown using decimal numbers, rather than using traditional hex numbers. In Figure C.1, the rightmost signals show the strongest RSS measured during the measurement, and the three strongest signals were received from MAC IDs 241001145012496, 241001145012498, and 241001145012499. Note that each AP has multiple interfaces and as such, a group of 


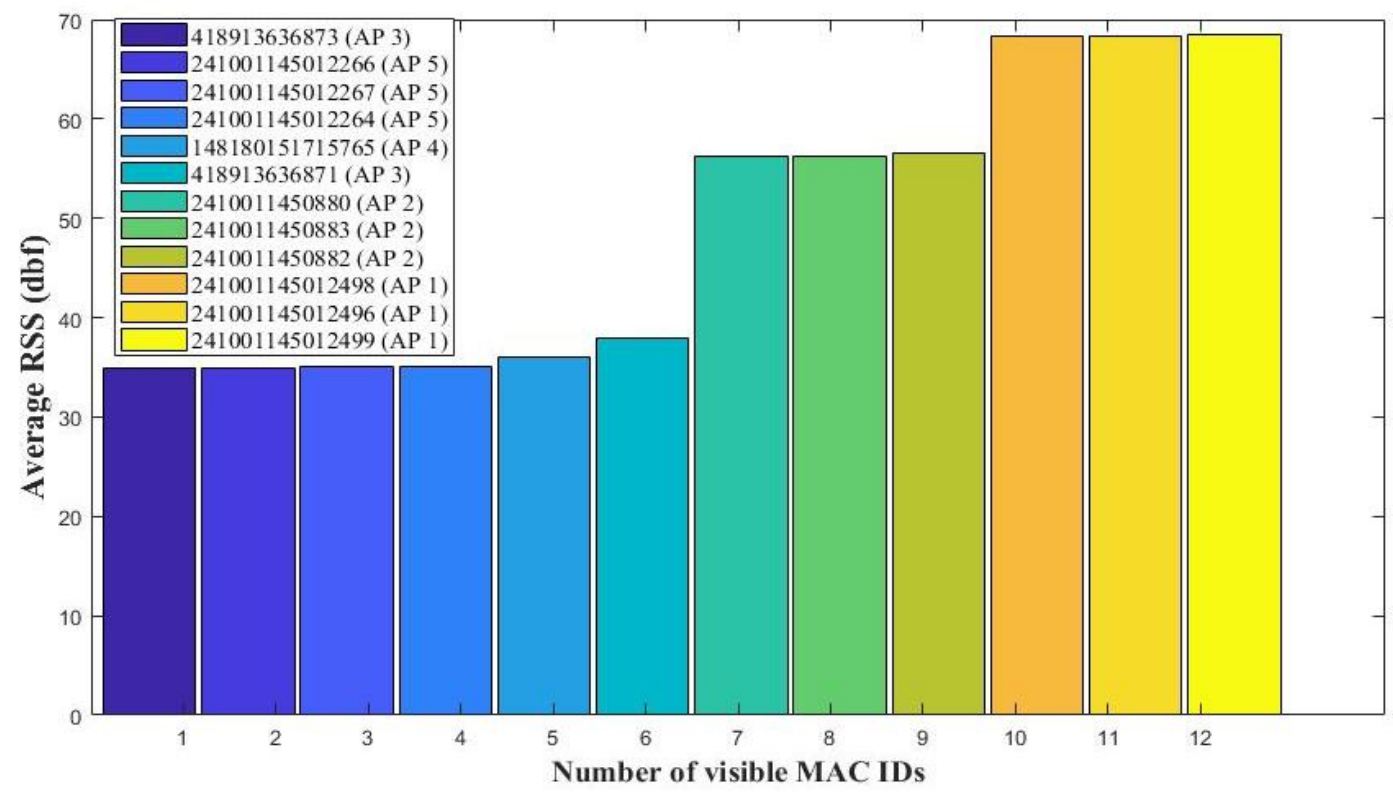

Figure C.1: Average RSS measurements

unique MAC IDs is assigned to it. The MAC IDs 241001145012496, 241001145012498, and 241001145012499 in the figure represent three interfaces of the same AP (shown as AP 1). Finally, these three IDs were considered as the addresses of the targeted AP, and second floor was selected as its location.

The process mentioned above in identifying the APs through site surveys follows a proximity-based approach, where it was assumed that on average, the strongest signal at a location is received from the AP with the shortest location-AP distance. For example, the distribution of RSS for the signals transmitted by AP 1 is shown in Figure C.2. The figure shows that the RSS readings for AP 1 from the second floor were higher than that of other floors. Also, there is a significant difference in the mean RSS of the two distributions. As such, second floor of MSC was selected as the floor location of AP 1.

Note that the above mentioned labor-intensive site survey was only required to identify the APs on each floor. As mentioned earlier in Chapter 4, the site survey for identifying APs can be simply avoided because the addresses and locations of the APs inside a building can be known from the assigned network engineers. For this dissertation, the locations of the APs inside ICT, MSC, and TFDL were verified by contacting The Information Technologies (IT) department of the University of Calgary. 


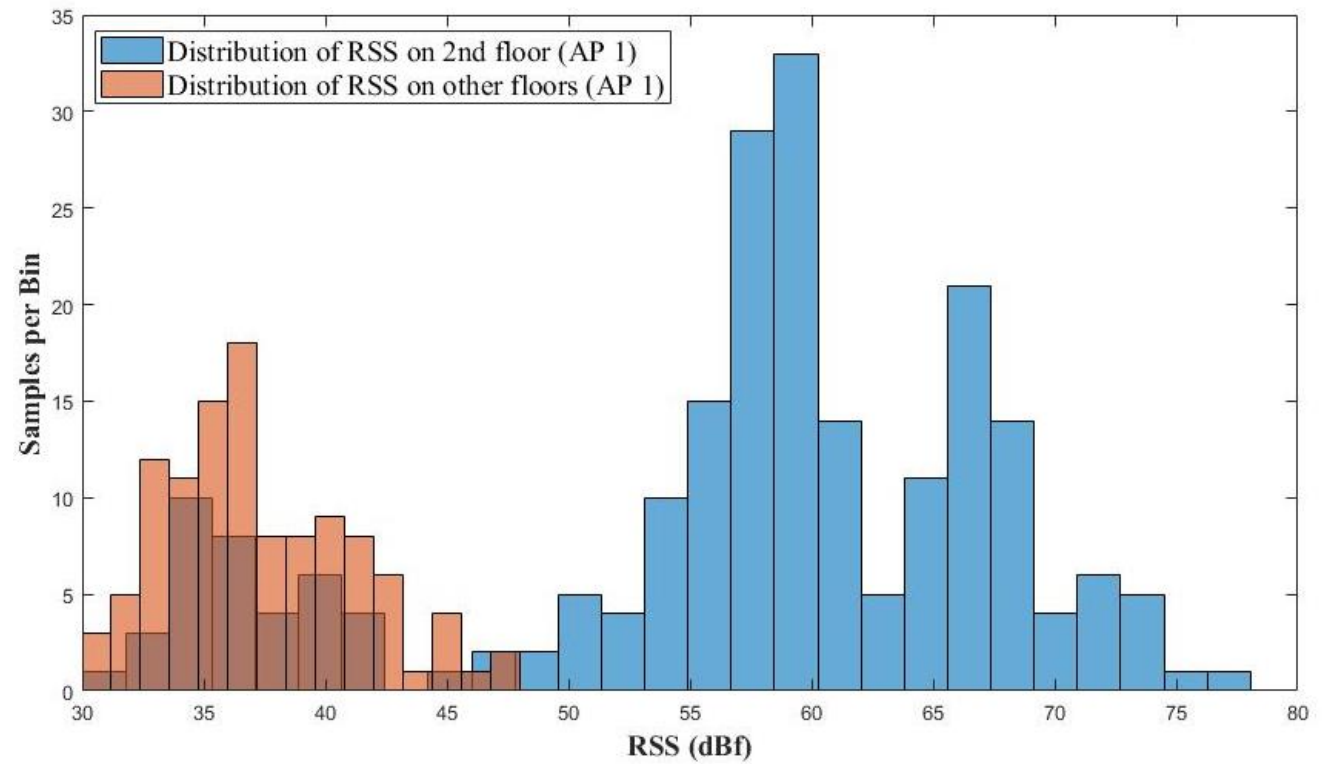

Figure C.2: Distribution of RSS on different floors (signals transmitted by AP 1) 


\section{IEEE COPYRIGHT FORM}

To ensure uniformity of treatment among all contributors, other forms may not be substituted for this form, nor may any wording of the form be changed. This form is intended for original material submitted to the IEEE and must accompany any such material in order to be published by the IEEE. Please read the form carefully and keep a copy for your files.

Wi-Fi RSS and MEMS Barometer Sensor Fusion Framework for Floor Localization

Haque, Fahimul; Dehghanian, Vahid; Fapojuwo, Abraham; Nielsen, John

IEEE Sensors Journal

\section{COPYRIGHT TRANSFER}

The undersigned hereby assigns to The Institute of Electrical and Electronics Engineers, Incorporated (the "IEEE") all rights under copyright that may exist in and to: (a) the Work, including any revised or expanded derivative works submitted to the IEEE by the undersigned based on the Work; and $(b)$ any associated written or multimedia components or other enhancements accompanying the Work.

\section{GENERAL TERMS}

1. The undersigned represents that he/she has the power and authority to make and execute this form.

2. The undersigned agrees to indemnify and hold harmless the IEEE from any damage or expense that may arise in the event of a breach of any of the warranties set forth above.

3. The undersigned agrees that publication with IEEE is subject to the policies and procedures of the IEEE PSPB Operations Manual.

4. In the event the above work is not accepted and published by the IEEE or is withdrawn by the author(s) before acceptance by the IEEE, the foregoing copyright transfer shall be null and void. In this case, IEEE will retain a copy of the manuscript for internal administrative/record-keeping purposes.

5. For jointly authored Works, all joint authors should sign, or one of the authors should sign as authorized agent for the others. 6. The author hereby warrants that the Work and Presentation (collectively, the "Materials") are original and that he/she is the author of the Materials. To the extent the Materials incorporate text passages, figures, data or other material from the works of others, the author has obtained any necessary permissions. Where necessary, the author has obtained all third party permissions and consents to grant the license above and has provided copies of such permissions and consents to IEEE

BY TYPING IN YOUR FULL NAME BELOW AND CLICKING THE SUBMIT BUTTON, YOU CERTIFY THAT SUCH ACTION CONSTITUTES YOUR ELECTRONIC SIGNATURE TO THIS FORM IN ACCORDANCE WITH UNITED STATES LAW, WHICH AUTHORIZES ELECTRONIC SIGNATURE BY AUTHENTICATED REQUEST FROM A USER OVER THE INTERNET AS A VALID SUBSTITUTE FOR A WRITTEN SIGNATURE.

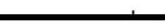

Signature
29-06-2018

Date (dd-mm-yyyy)

\section{Information for Authors}

\section{AUTHOR RESPONSIBILITIES}

The IEEE distributes its technical publications throughout the world and wants to ensure that the material submitted to its publications is properly available to the readership of those publications. Authors must ensure that their Work meets the requirements as stated in section 8.2.1 of the IEEE PSPB Operations Manual, including provisions covering originality, 
authorship, author responsibilities and author misconduct. More information on IEEE's publishing policies may be found at http://www.ieee.org/publications standards/publications/rights/authorrightsresponsibilities.html Authors are advised especially of IEEE PSPB Operations Manual section 8.2.1.B12: "It is the responsibility of the authors, not the IEEE, to determine whether disclosure of their material requires the prior consent of other parties and, if so, to obtain it." Authors are also advised of IEEE PSPB Operations Manual section 8.1.1B: "Statements and opinions given in work published by the IEEE are the expression of the authors."

\section{RETAINED RIGHTS/TERMS AND CONDITIONS}

- Authors/employers retain all proprietary rights in any process, procedure, or article of manufacture described in the Work.

- Authors/employers may reproduce or authorize others to reproduce the Work, material extracted verbatim from the Work, or derivative works for the author's personal use or for company use, provided that the source and the IEEE copyright notice are indicated, the copies are not used in any way that implies IEEE endorsement of a product or service of any employer, and the copies themselves are not offered for sale.

- Although authors are permitted to re-use all or portions of the Work in other works, this does not include granting third-party requests for reprinting, republishing, or other types of re-use.The IEEE Intellectual Property Rights office must handle all such third-party requests.

- Authors whose work was performed under a grant from a government funding agency are free to fulfill any deposit mandates from that funding agency.

\section{AUTHOR ONLINE USE}

- Personal Servers. Authors and/or their employers shall have the right to post the accepted version of IEEE-copyrighted articles on their own personal servers or the servers of their institutions or employers without permission from IEEE, provided that the posted version includes a prominently displayed IEEE copyright notice and, when published, a full citation to the original IEEE publication, including a link to the article abstract in IEEE Xplore. Authors shall not post the final, published versions of their papers.

- Classroom or Internal Training Use. An author is expressly permitted to post any portion of the accepted version of his/her own IEEE-copyrighted articles on the author's personal web site or the servers of the author's institution or company in connection with the author's teaching, training, or work responsibilities, provided that the appropriate copyright, credit, and reuse notices appear prominently with the posted material. Examples of permitted uses are lecture materials, course packs, ereserves, conference presentations, or in-house training courses.

- Electronic Preprints. Before submitting an article to an IEEE publication, authors frequently post their manuscripts to their own web site, their employer's site, or to another server that invites constructive comment from colleagues. Upon submission of an article to IEEE, an author is required to transfer copyright in the article to IEEE, and the author must update any previously posted version of the article with a prominently displayed IEEE copyright notice. Upon publication of an article by the IEEE, the author must replace any previously posted electronic versions of the article with either (1) the full citation to the IEEE work with a Digital Object Identifier (DOI) or link to the article abstract in IEEE Xplore, or (2) the accepted version only (not the IEEE-published version), including the IEEE copyright notice and full citation, with a link to the final, published article in IEEE Xplore.

Questions about the submission of the form or manuscript must be sent to the publication's editor.

Please direct all questions about IEEE copyright policy to:

IEEE Intellectual Property Rights Office, copyrights@ieee.org, +1-732-562-3966 


\section{IEEE COPYRIGHT AND CONSENT FORM}

To ensure uniformity of treatment among all contributors, other forms may not be substituted for this form, nor may any wording of the form be changed. This form is intended for original material submitted to the IEEE and must accompany any such material in order to be published by the IEEE. Please read the form carefully and keep a copy for your files.

TITLE OF PAPER/ARTICLE/REPORT, INCLUDING ALL CONTENT IN ANY FORM, FORMAT, OR MEDIA (hereinafter, "the Work"): Sensor Fusion for Floor Detection

COMPLETE LIST OF AUTHORS: Fahimul Haque, Vahid Dehghanian, Abraham O. Fapojuwo

\section{IEEE PUBLICATION TITLE (Journal, Magazine, Conference, Book): The 8th IEEE Annual Information Technology, Electronics and Mobile Communication Conference (IEEE IEMCON 2017) \\ COPYRIGHT TRANSFER}

1. The undersigned hereby assigns to The Institute of Electrical and Electronics Engineers, Incorporated (the "IEEE") all rights under copyright that may exist in and to: (a) the above Work, including any revised or expanded derivative works submitted to the IEEE by the undersigned based on the Work; and (b) any associated written or multimedia components or other enhancements accompanying the Work.

\section{CONSENT AND RELEASE}

2. In the event the undersigned makes a presentation based upon the Work at a conference hosted or sponsored in whole or in part by the IEEE, the undersigned, in consideration for his/her participation in the conference, hereby grants the IEEE the unlimited, worldwide, irrevocable permission to use, distribute, publish, license, exhibit, record, digitize, broadcast, reproduce and archive, in any format or medium, whether now known or hereafter developed: (a) his/her presentation and comments at the conference; (b) any written materials or multimedia files used in connection with his/her presentation; and (c) any recorded interviews of him/her (collectively, the "Presentation"). The permission granted includes the transcription and reproduction of the Presentation for inclusion in products sold or distributed by IEEE and live or recorded broadcast of the Presentation during or after the conference.

3. In connection with the permission granted in Section 2, the undersigned hereby grants IEEE the unlimited, worldwide, irrevocable right to use his/her name, picture likeness, voice and biographical information as part of the advertisement, distribution and sale of products incorporating the Work or Presentation, and releases IEEE from any claim based on right of privacy or publicity.

4. The undersigned hereby warrants that the Work and Presentation (collectively, the "Materials") are original and that he/she is the author of the Materials. To the extent the Materials incorporate text passages, figures, data or other material from the works of others, the undersigned has obtained any necessary permissions. Where necessary, the undersigned has obtained all third party permissions and consents to grant the license above and has provided copies of such permissions and consents to IEEE

$\square$ Please check this box if you do not wish to have video/audio recordings made of your conference presentation See reverse side for Retained Rights/Terms and Conditions, and Author Responsibilities.

\section{GENERAL TERMS}

- The undersigned represents that he/she has the power and authority to make and execute this assignment.

- The undersigned agrees to indemnify and hold harmless the IEEE from any damage or expense that may arise in the event of a breach of any of the warranties set forth above.

- In the event the above work is not accepted and published by the IEEE or is withdrawn by the author(s) before acceptance by the IEEE, the foregoing copyright transfer shall become null and void. Even in this case, IEEE will retain an archival copy of the manuscript.

- For jointly authored Works, all joint authors should sign, or one of the authors should sign as authorized agent for the others.

(1)

Author/Authorized Agent for Joint Authors

22 August, 2017

Date

\section{U.S. GOVERNMENT EMPLOYEE CERTIFICATION (WHERE APPLICABLE)}

This will certify that all authors of the Work are U.S. government employees and prepared the Work on a subject within the scope of their official duties. As such, the Work is not subject to U.S. copyright protection.

(2)

Authorized Signature

Date

(Authors who are U.S. government employees should also sign signature line (1) above to enable the IEEE to claim and protect its copyright in international jurisdictions.)

CROWN COPYRIGHT CERTIFICATION (WHERE APPLICABLE)

This will certify that all authors of the Work are employees of the British or British Commonwealth Government and prepared the Work in connection with their officia duties. As such, the Work is subject to Crown Copyright and is not assigned to the IEEE as set forth in the first sentence of the Copyright Transfer Section above. The undersigned acknowledges, however, that the IEEE has the right to publish, distribute and reprint the Work in all forms and media.

(3)

Authorized Signature

(Authors who are British or British Commonwealth Government employees should also sign line (1) above to indicate their acceptance of all terms other than the copyright transfer.) 


\section{IEEE COPYRIGHT FORM (continued)}

\section{RETAINED RIGHTS/TERMS AND CONDITIONS}

\section{General}

1. Authors/employers retain all proprietary rights in any process, procedure, or article of manufacture described in the Work.

2. Authors/employers may reproduce or authorize others to reproduce the Work, material extracted verbatim from the Work, or derivative works for the author's personal use or for company use, provided that the source and the IEEE copyright notice are indicated, the copies are not used in any way that implies IEEE endorsement of a product or service of any employer, and the copies themselves are not offered for sale.

3. In the case of a Work performed under a U.S. Government contract or grant, the IEEE recognizes that the U.S. Government has royalty-free permission to reproduce all or portions of the Work, and to authorize others to do so, for official U.S. Government purposes only, if the contract/grant so requires.

4. Although authors are permitted to re-use all or portions of the Work in other works, this does not include granting third-party requests for reprinting, republishing, or other types of re-use. The IEEE Intellectual Property Rights office must handle all such third-party requests.

5. Authors whose work was performed under a grant from a government funding agency are free to fulfill any deposit mandates from that funding agency.

\section{Author Online Use}

6. Personal Servers. Authors and/or their employers shall have the right to post the accepted version of IEEE-copyrighted articles on their own personal servers or the servers of their institutions or employers without permission from IEEE, provided that the posted version includes a prominently displayed IEEE copyright notice and, when published, a full citation to the original IEEE publication, including a link to the article abstract in IEEE Xplore. Authors shall not post the final, published versions of their papers.

7. Classroom or Internal Training Use. An author is expressly permitted to post any portion of the accepted version of his/her own IEEE-copyrighted articles on the author's personal web site or the servers of the author's institution or company in connection with the author's teaching, training, or work responsibilities, provided that the appropriate copyright, credit, and reuse notices appear prominently with the posted material. Examples of permitted uses are lecture materials, course packs, e-reserves, conference presentations, or in-house training courses.

8. Electronic Preprints. Before submitting an article to an IEEE publication, authors frequently post their manuscripts to their own web site, their employer's site, or to another server that invites constructive comment from colleagues. Upon submission of an article to IEEE, an author is required to transfer copyright in the article to IEEE, and the author must update any previously posted version of the article with a prominently displayed IEEE copyright notice. Upon publication of an article by the IEEE, the author must replace any previously posted electronic versions of the article with either (1) the full citation to the IEEE work with a Digital Object Identifier (DOI) or link to the article abstract in IEEE Xplore, or (2) the accepted version only (not the IEEE-published version), including the IEEE copyright notice and full citation, with a link to the final, published article in IEEE Xplore.

\section{Author Responsibilities}

\section{INFORMATION FOR AUTHORS}

The IEEE distributes its technical publications throughout the world and wants to ensure that the material submitted to its publications is properly available to the readership of those publications. Authors must ensure that their Work meets the requirements as stated in section 8.2.1 of the IEEE PSPB Operations Manual, including provisions covering originality, authorship, author responsibilities and author misconduct. More information on IEEE's publishing policies may be found at http://www.ieee.org/publications standards/publications/rights/authorrightsresponsibilities.html. Authors are advised especially of IEEE PSPB Operations Manual section 8.2.1.B12: "It is the responsibility of the authors, not the IEEE, to determine whether disclosure of their material requires the prior consent of other parties and, if so, to obtain it." Authors are also advised of IEEE PSPB Operations Manual section 8.1.1B: "Statements and opinions given in work published by the IEEE are the expression of the authors."

\section{Author/Emplover Rights}

If you are employed and prepared the Work on a subject within the scope of your employment, the copyright in the Work belongs to your employer as a work-for-hire. In that case, the IEEE assumes that when you sign this Form, you are authorized to do so by your employer and that your employer has consented to the transfer of copyright, to the representation and warranty of publication rights, and to all other terms and conditions of this Form. If such authorization and consent has not been given to you, an authorized representative of your employer should sign this Form as the Author.

IEEE Copyright Ownership

It is the formal policy of the IEEE to own the copyrights to all copyrightable material in its technical publications and to the individual contributions contained therein, in order to protect the interests of the IEEE, its authors and their employers, and, at the same time, to facilitate the appropriate re-use of this material by others. The IEEE distributes its technical publications throughout the world and does so by various means such as hard copy, microfiche, microfilm, and electronic media. It also abstracts and may translate its publications, and articles contained therein, for inclusion in various compendiums, collective works, databases and similar publications.

THIS FORM MUST ACCOMPANY THE SUBMISSION OF THE AUTHOR'S MANUSCRIPT.

Questions about the submission of the form or manuscript must be sent to the publication's editor.

Please direct all questions about IEEE copyright policy to:

IEEE Intellectual Property Rights Office, copvrights@ieee.org, +1-732-562-3966 (telephone) 